\title{
Ring-Modified Analogues and Molecular Dynamics Studies to Probe the Requirements for Fungicidal Activities of Malayamycin A and Its $N$-Nucleoside Variants
}

\section{- Supporting Information}

\section{Contents:}

Compound characterization list..................................S2

General Experimental..............................................S3

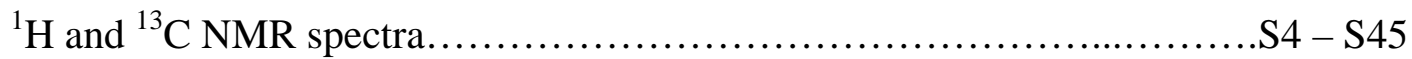

X-ray crystallographic data for compound 18......................S46 - S47

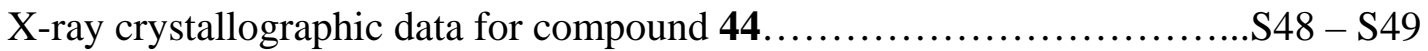

Tables for energies and HF calculations for 7, 42, 43.................S50 - S64 


\section{Compound}

or structure number

(with

substituent/ isomer identifier if needed)
Known compound [citation given]

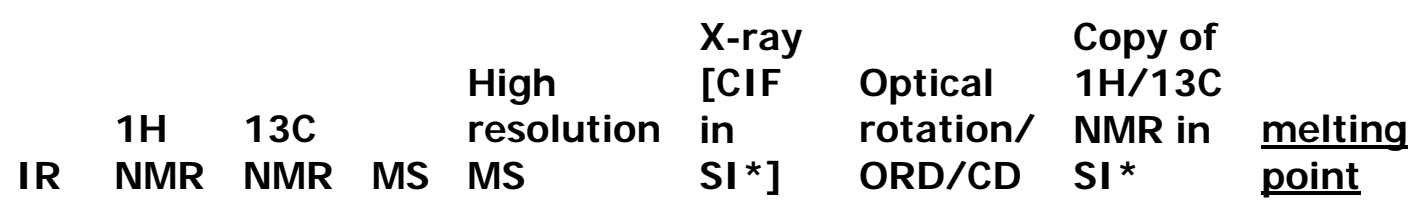

X-ray

Optical
ORD/ CD

point

\begin{tabular}{c|c|c|c|c|c|c|c}
$\mathbf{X}$ & $\mathbf{X}$ & $\mathbf{X}$ & & & $\mathbf{X}$ & $\mathbf{X}$ & $\mathbf{X}$ \\
$\mathbf{X}$ & $\mathbf{X}$ & & $\mathbf{X}$ & & $\mathbf{X}$ & $\mathbf{X}$ & $\mathbf{X}$ \\
\hline
\end{tabular}

\begin{tabular}{l|l|} 
& $\mathrm{x}$ \\
& $\mathrm{x}$
\end{tabular}

$\mathrm{X}$

17

18

19

21

22

10

29

30

31

32

36

37

38

39

40

11

\begin{tabular}{|c|c|} 
& \\
\hline$x$ & \\
\hline$x$ & \\
\hline$X$ & $x$ \\
\hline$X$ & \\
\hline & \\
\hline
\end{tabular}

$\begin{array}{ll}X & X\end{array}$

\begin{tabular}{l|l|l|}
$\mathrm{X}$ & $\mathrm{X}$ \\
$\mathrm{X}$ & & $\mathrm{x}$ \\
\hline
\end{tabular}

\begin{tabular}{l|l}
$\mathrm{x}$ & $\mathrm{x}$ \\
$\mathrm{x}$ & $\mathrm{x}$
\end{tabular}

$\mathbf{X} \quad \mathrm{X}$

\begin{tabular}{l|l}
$x$ & $x$ \\
\hline$x$ & $x$
\end{tabular}

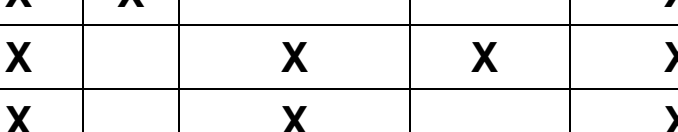

\begin{tabular}{l|l}
$\mathrm{x}$ & $\mathrm{x}$ \\
$\mathrm{x}$ & $\mathrm{x}$ \\
\hline
\end{tabular}

\begin{tabular}{l|l}
$\mathrm{x}$ & $\mathrm{x}$ \\
$\mathrm{x}$ & $\mathrm{x}$
\end{tabular}

\begin{tabular}{l|l}
$x$ & $x$ \\
\hline
\end{tabular}




\section{General Experimental}

Anhydrous solvents were transferred under a positive pressure of Ar from a solvent dispensing system. All commercially available reagents were used without further purification. NMR spectra were reported in parts per million (ppm) downfield from tetramethylsilane, as an internal reference, and coupling constants $(J)$ were reported in Hertz. Either protonated molecular ions $[\mathrm{M}+\mathrm{H}]^{+}$or sodium adducts $[\mathrm{M}+\mathrm{Na}]^{+}$were used for emprical ion confirmation. Melting points are uncorrected. Optical roations were recorded in a $1 \mathrm{dm}$ cell at ambient temperature with a sodium lamp (wavelength of 589 $\mathrm{nm})$. Analytical thin-layer chromatography was performed on $60 \mathrm{~F}_{254}$ pre-coated silica gel plates. Visualization was achieved using ultraviolet light and/or staining with ceric ammonium molybdate or ninhydrin. Flash chromatography was performed on a column of 230-400 mesh silica gel with the indicated solvent system. 


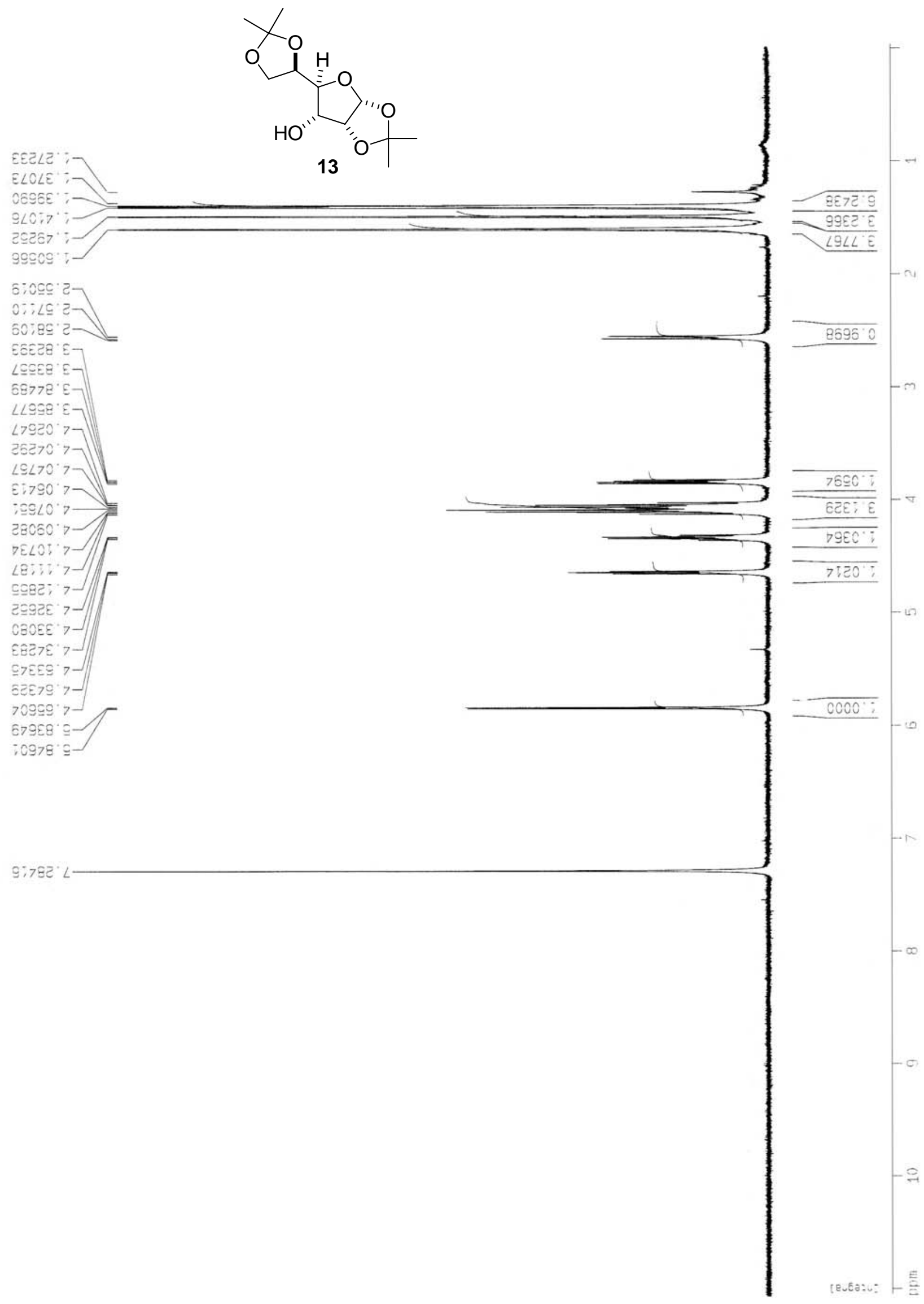


$888^{\circ} \nabla 2$

टट6 92

61192

$261 \cdot 92$

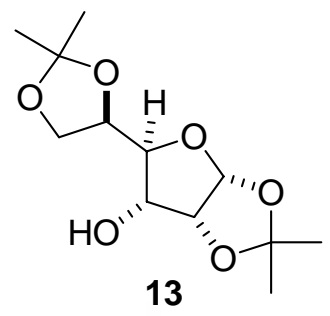

EटV $99-$

$900^{\circ} \mathrm{CL}$

901. $9 /$

66ह $9 L$

$\angle L^{\circ} 9 L$

$\triangle 85 \cdot 86-$

हणट $6 L]$

ธ० द $\mathcal{\varepsilon} \mathrm{I}$

$090^{\circ} 601-$

200.210 

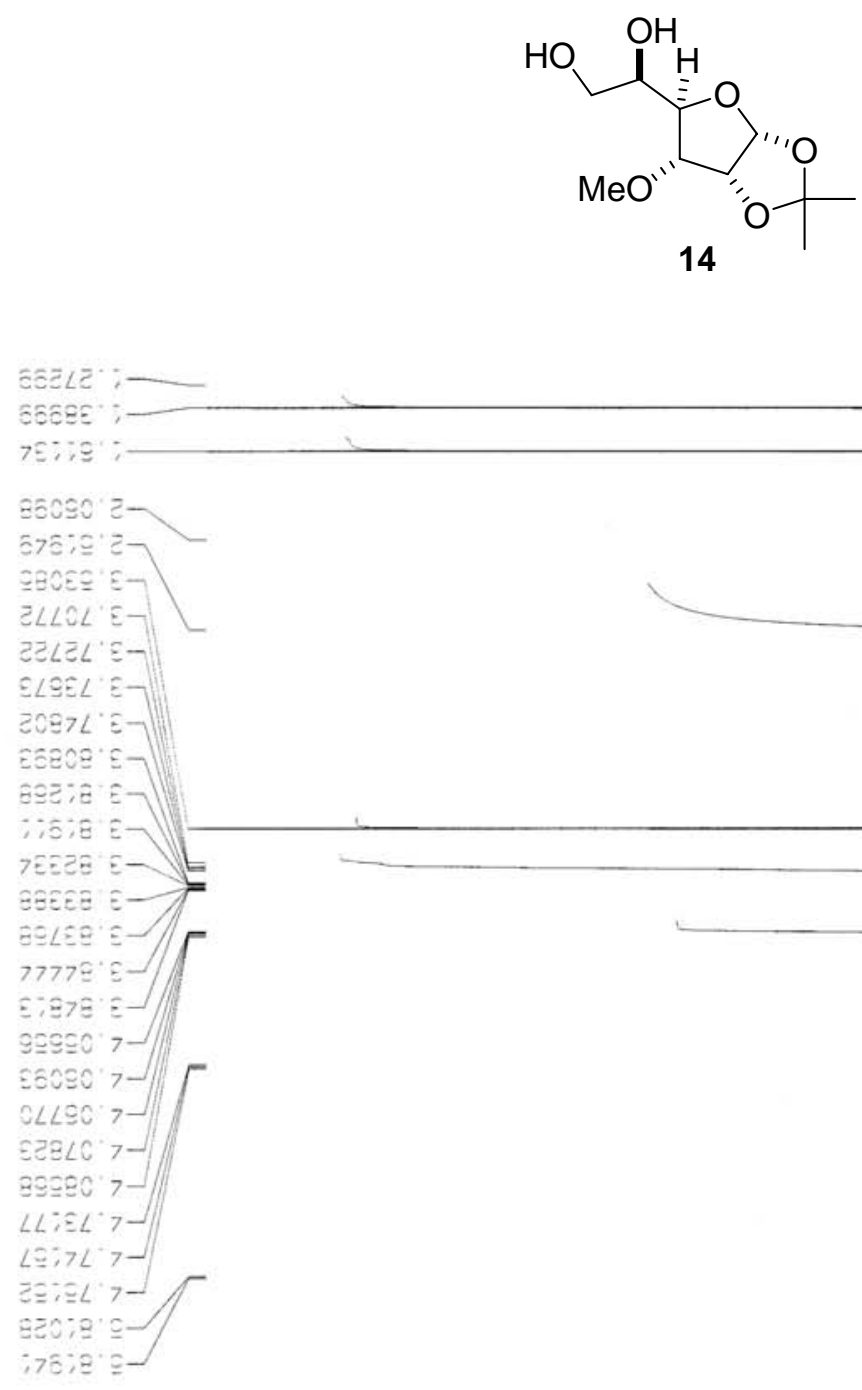

geese $L-$
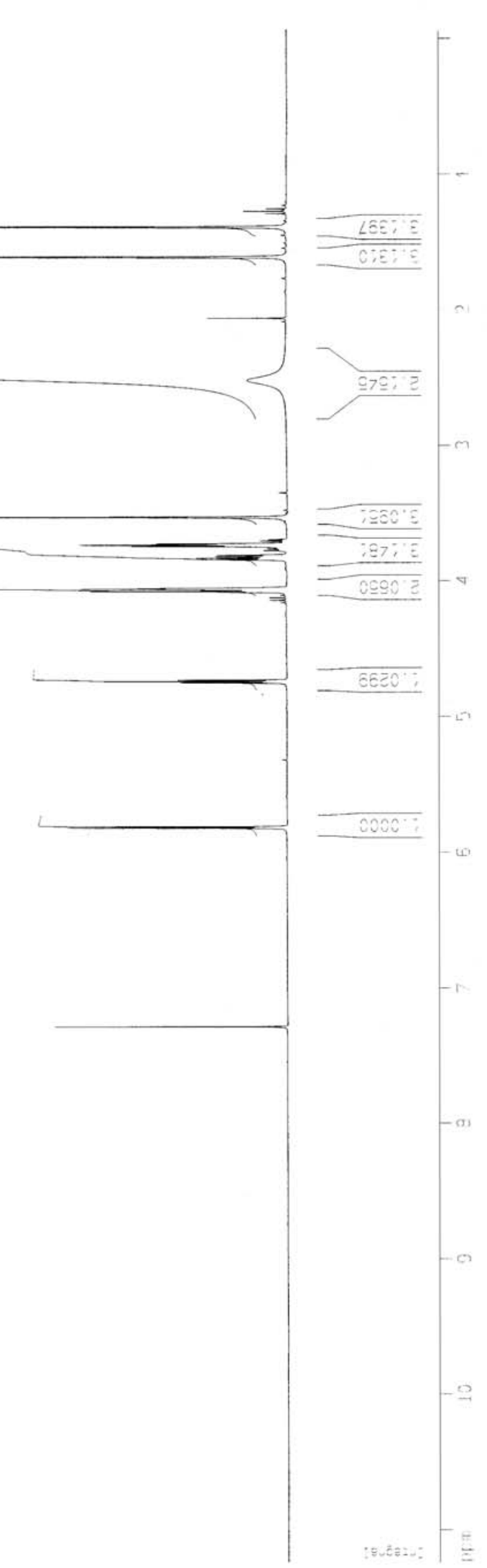


$$
4
$$



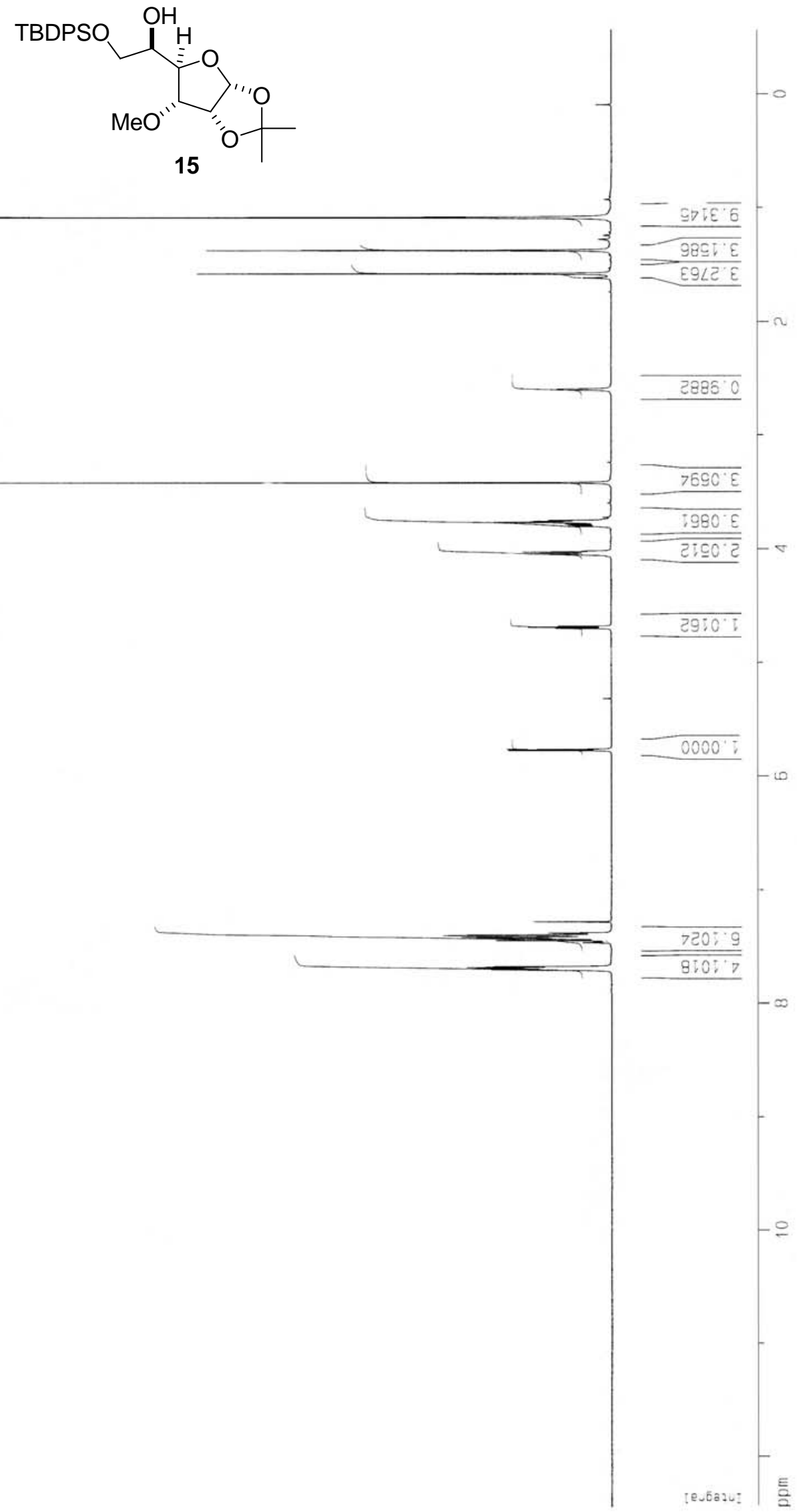


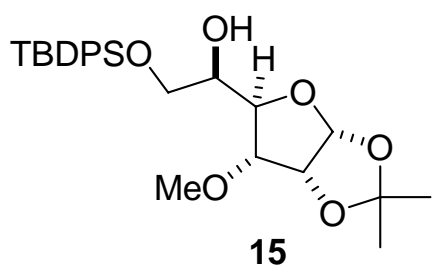

$\varepsilon 88^{\circ} 81-$
$\varepsilon \angle V^{\circ} 92$
$\angle 7 \nabla^{\circ} 92$
$\angle \nabla^{\circ} 92$

$\operatorname{ID9} \angle G-$

$100^{\circ} \nabla 9$

9EG $L L$

8EE' $9 L$

$999^{\circ} 9 L$

ट $\angle 6.9 \angle$

$\angle I C^{\prime} \angle L$

टD0.08-

Eเ9'E0I

LE9 ट⿱一兀

$\varepsilon 9 \varepsilon \angle 2 t$

$\nabla \angle E^{\circ} \angle 2 !$

$89 \varepsilon^{\circ}$ 6己t

टO० 621

99L टहा-

008 'टE
$\angle 02$ 'GEI

ODC'GE:-

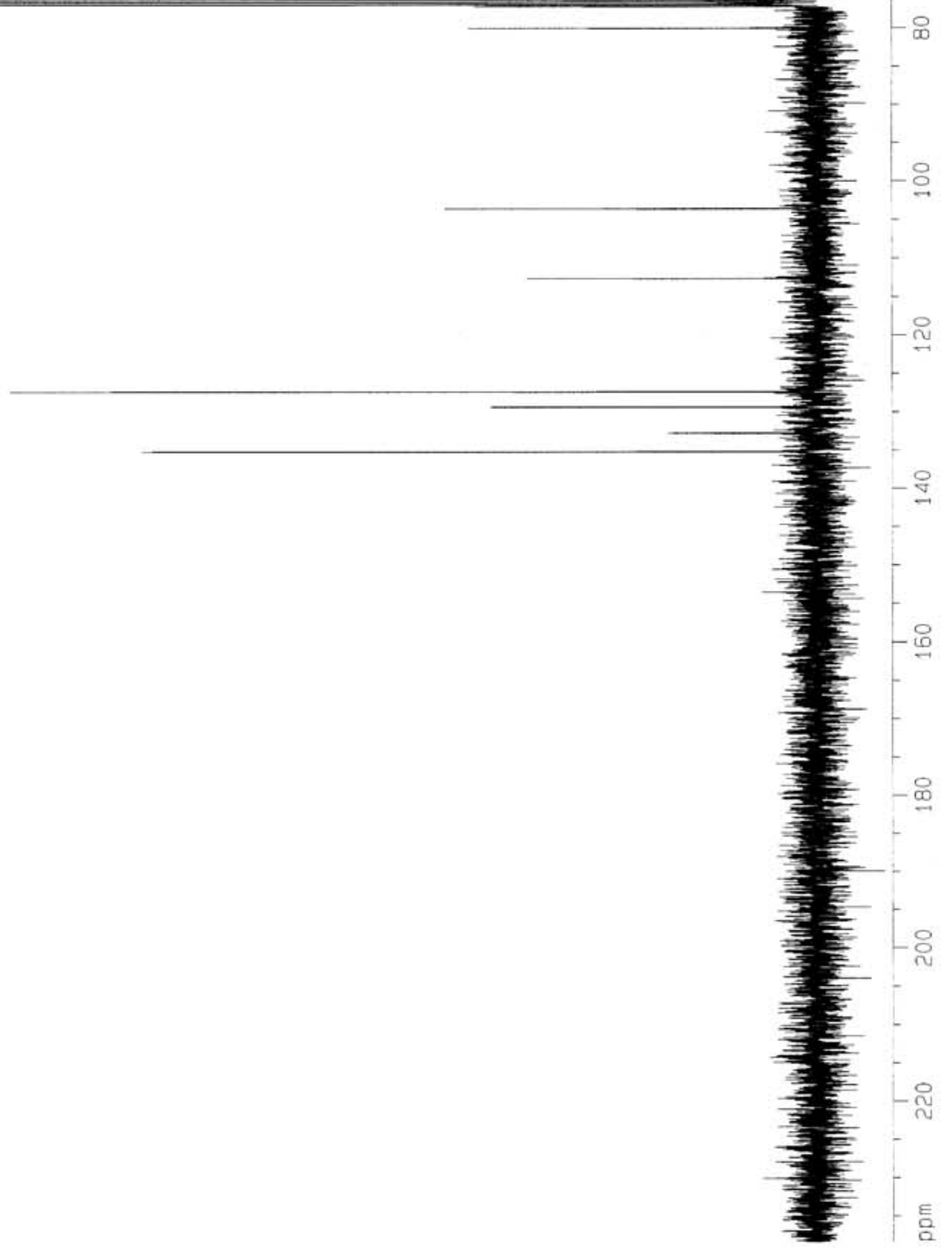




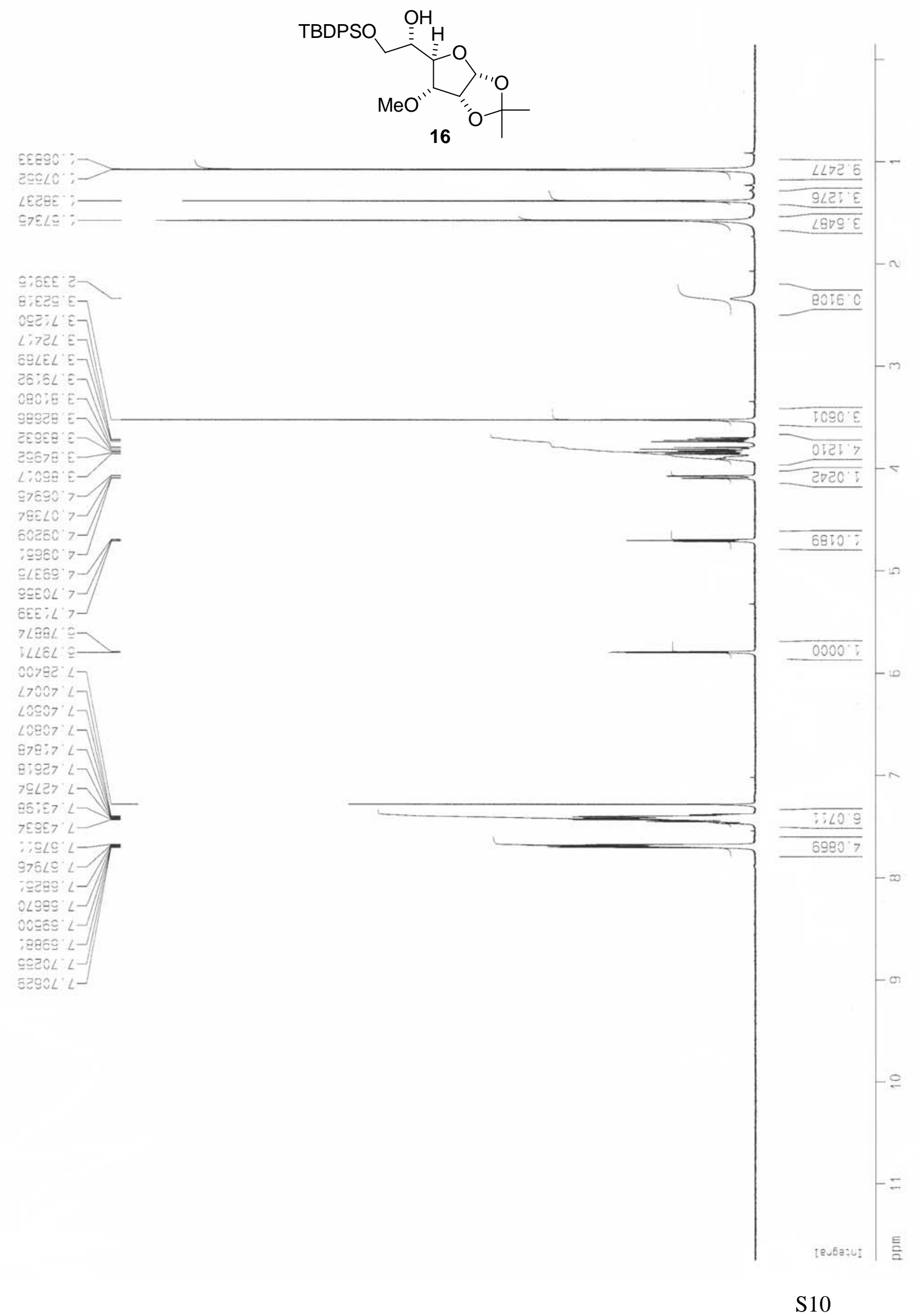




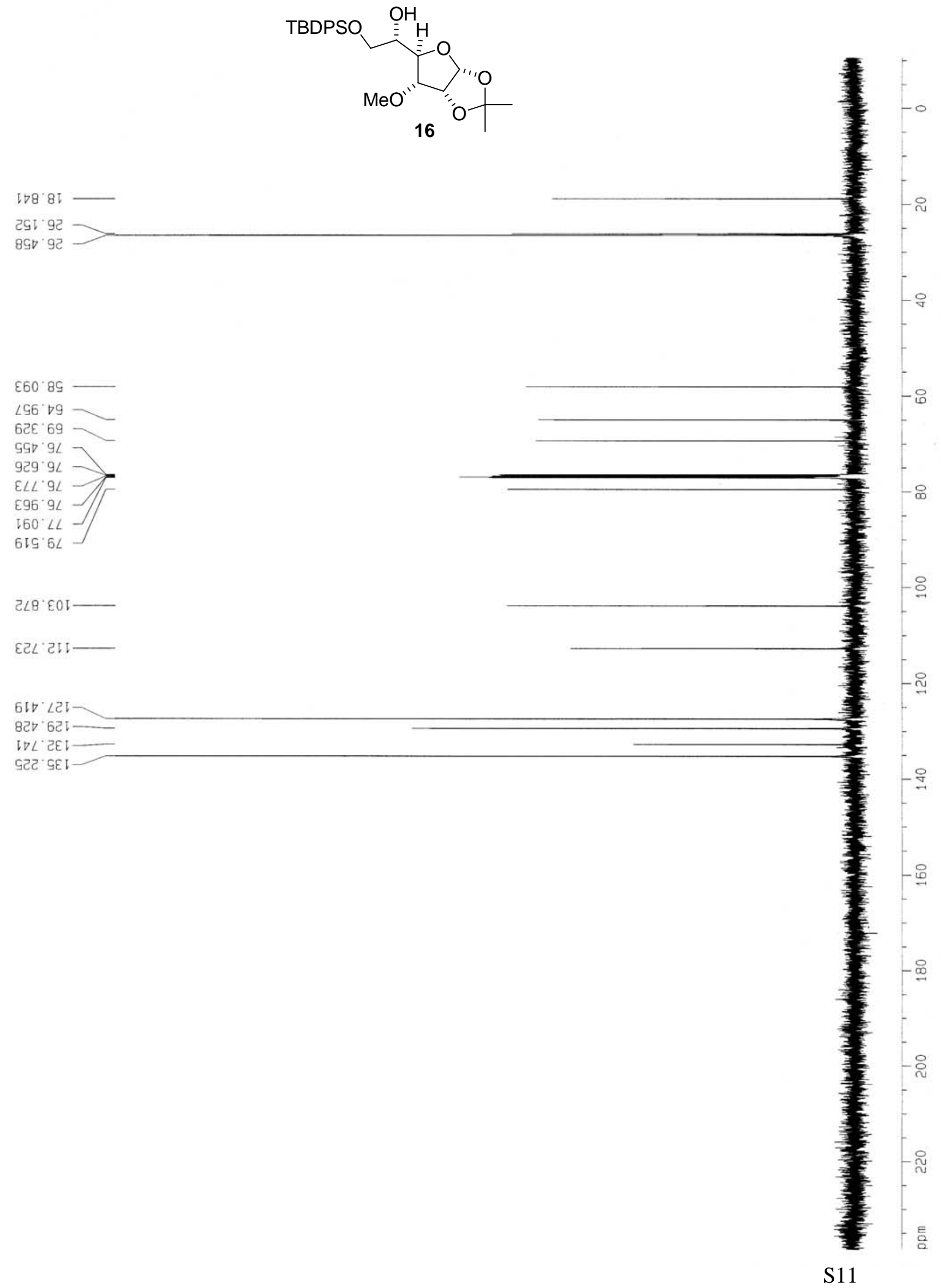




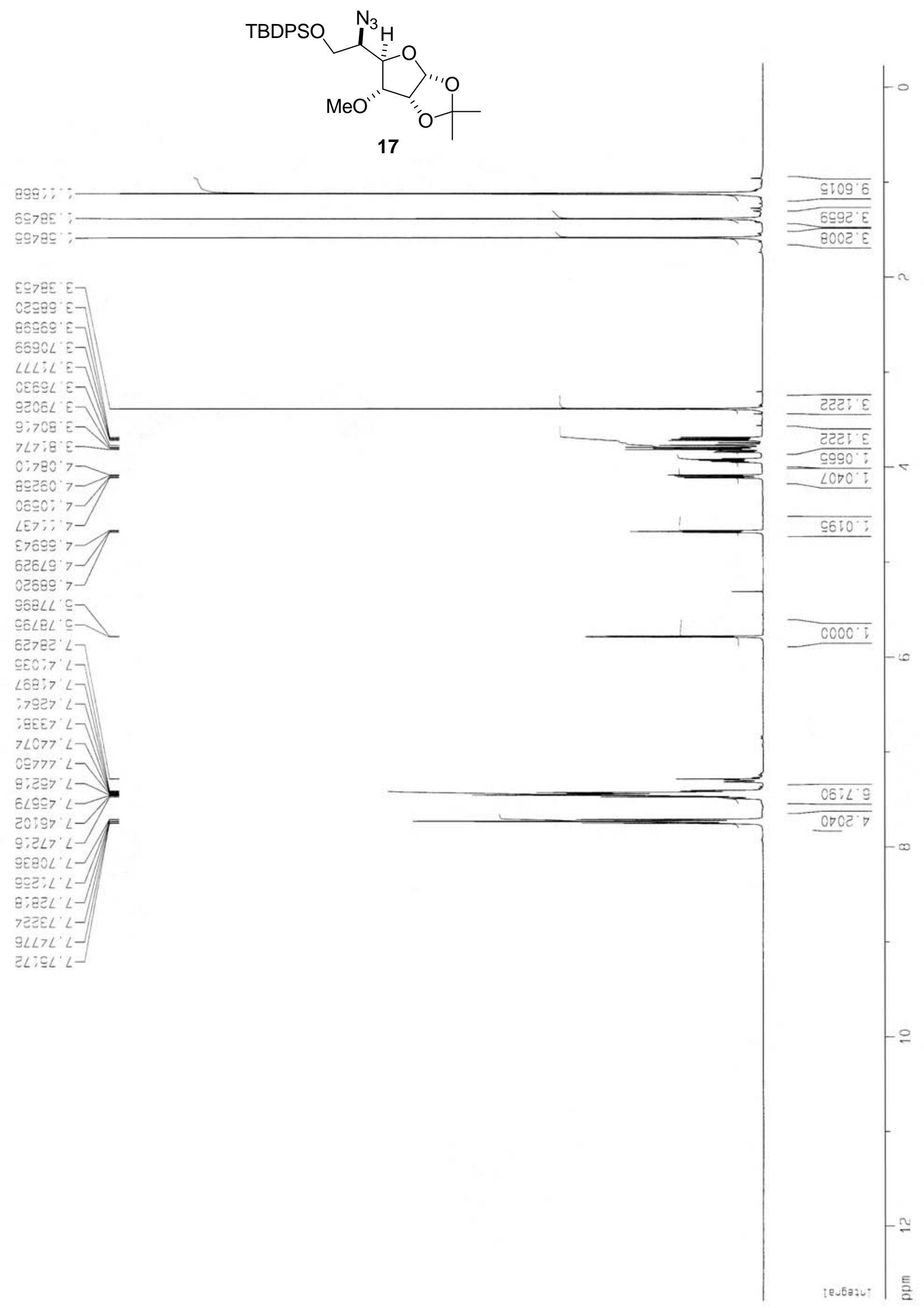




$$
\text { | }
$$


$87890^{\circ} 0$
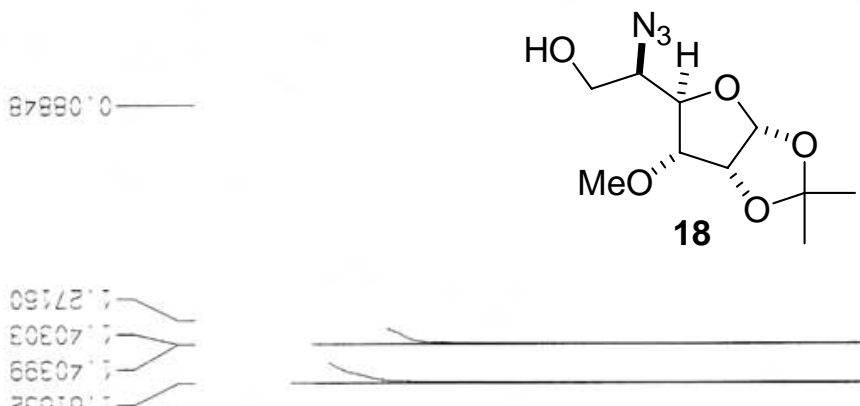

zeg:9 :-

วอยฐ $\varepsilon-$

E:8EL $E-$

$; 9 ;>L$

EEESE E-

$70798 \varepsilon-$

$7 Z \subseteq \angle \theta^{\prime} \varepsilon-$

อระอ8 E-

ธะ766 $\varepsilon-$

$7 \subseteq 100 \div$

EE $\angle O O^{\circ} 7=$

$\therefore \angle 7: 0{ }^{\circ}>$

ELOJO $7-1$

$76 \angle \mathrm{LO}^{\circ}{ }^{-1}$

7हi浮7

海浜 7 -

รEEes $7-$

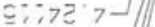

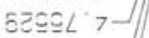

$\Xi \angle 7 \Xi \angle>-$

$\angle 67 L L 7=$

68778. $\cong-$

LeEgQ'

$27782^{\circ} L$

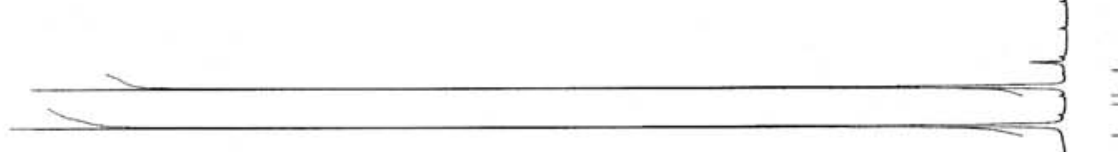

$\frac{8897^{\circ} \varepsilon}{2 E 95 .}$
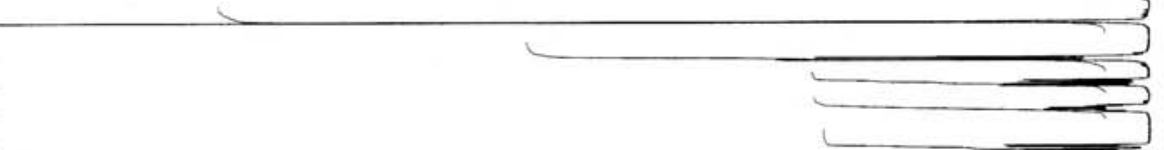

$=\operatorname{sgc0} 0^{\circ}$

$7070^{\circ}$

ESEO :

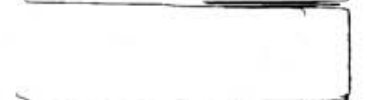

$0666^{\circ}$

टE20
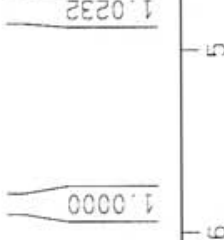

$-\infty$

$-\infty$

웅

eusazuI 


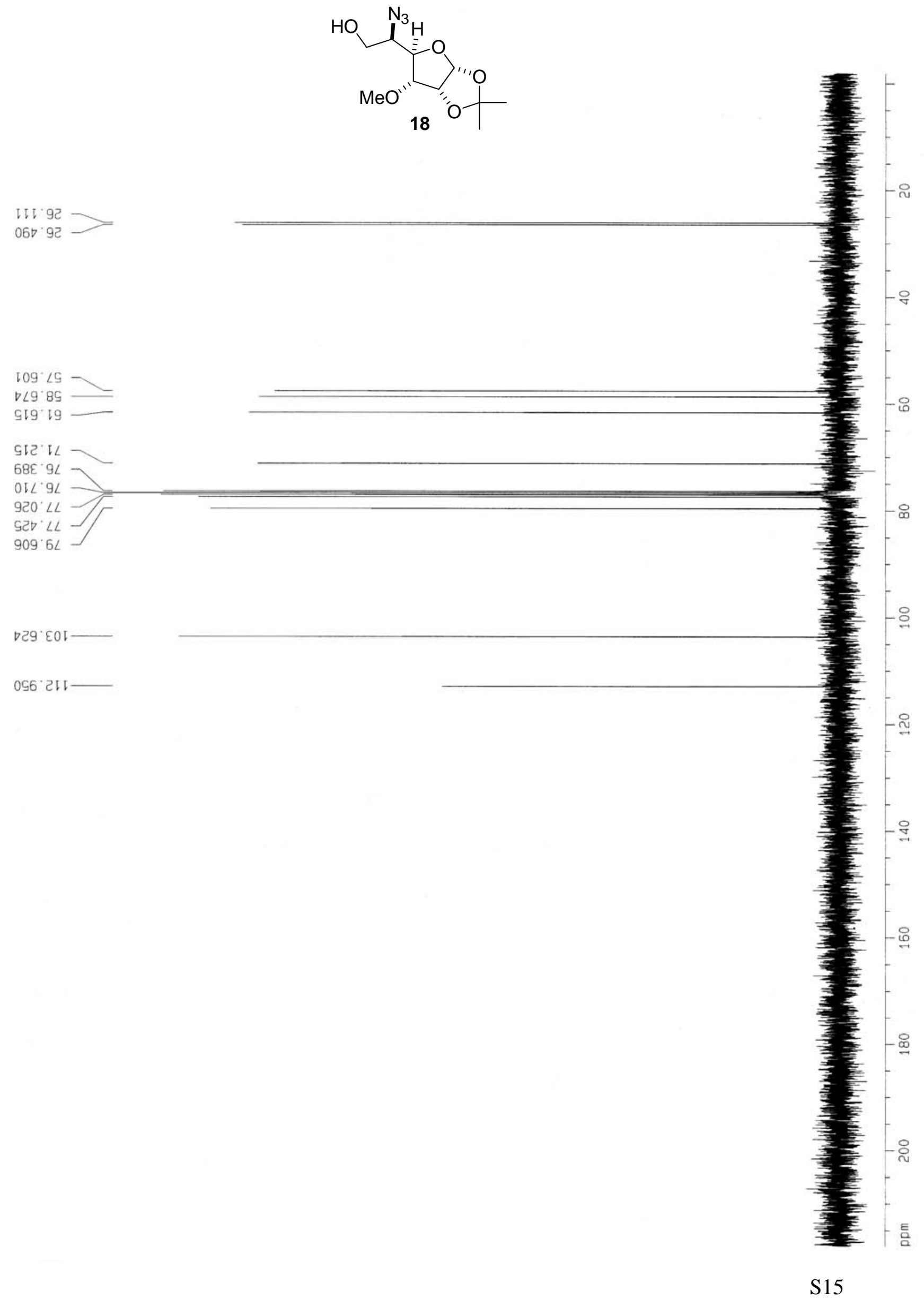



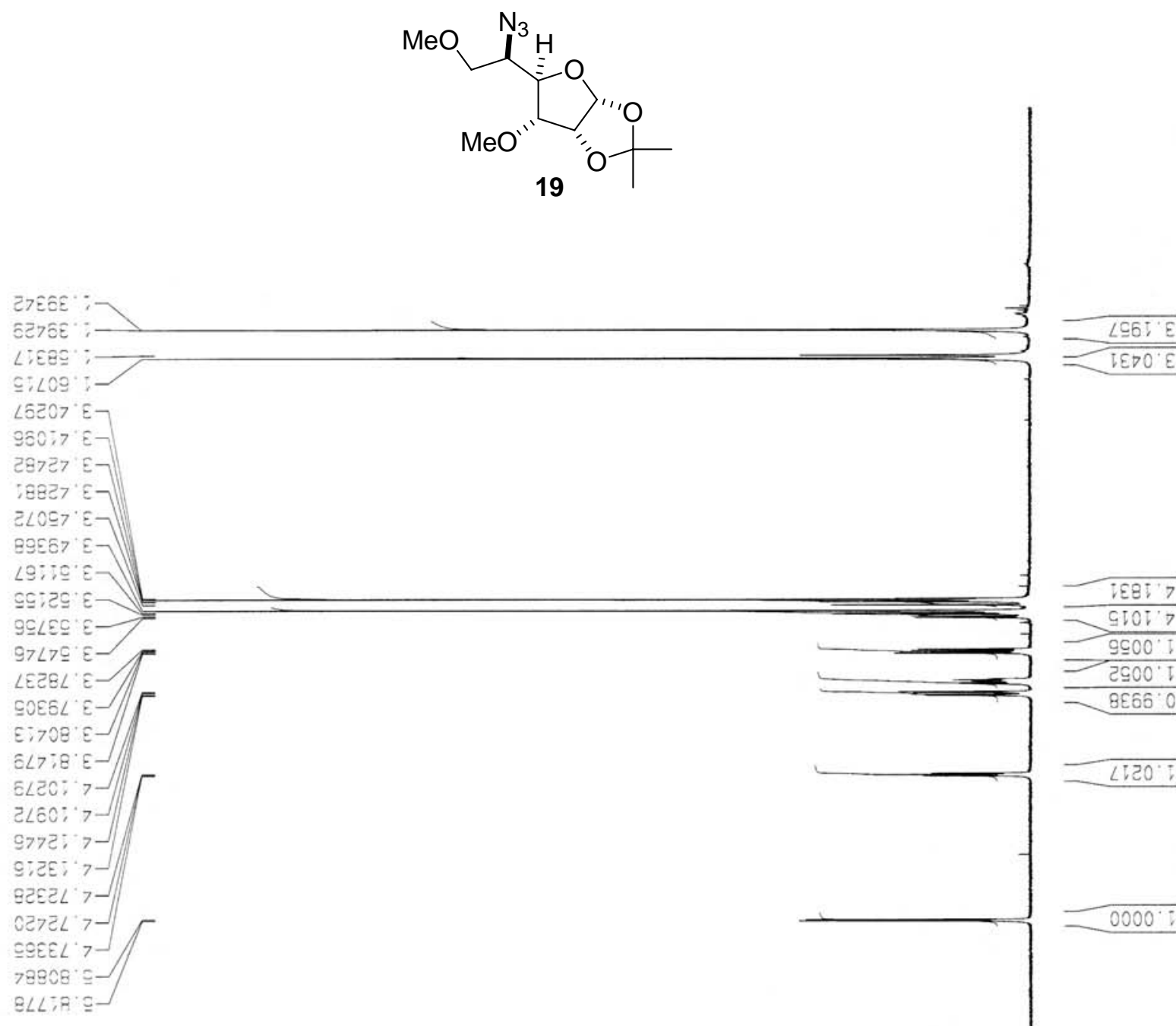

7,782 


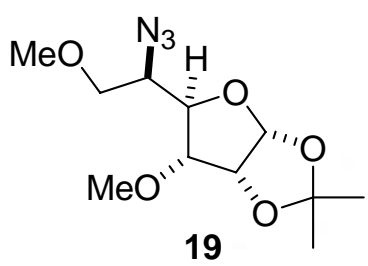

6EI.92

LIG $92-$

$\angle E g^{\circ} \angle G$

$80 \angle 89 \longrightarrow$

हE9. I9 -

$\angle \nabla C^{\prime} L$

$\angle E E^{\prime} 9 L$

५५9 $9 /$

9EL' $9 L$

$2 \angle 6^{\circ} 9 \angle$

89० $L$

GE9.6L

G७ $9^{\circ} \varepsilon 0 \mathrm{~L}$

$266^{\circ} 211$

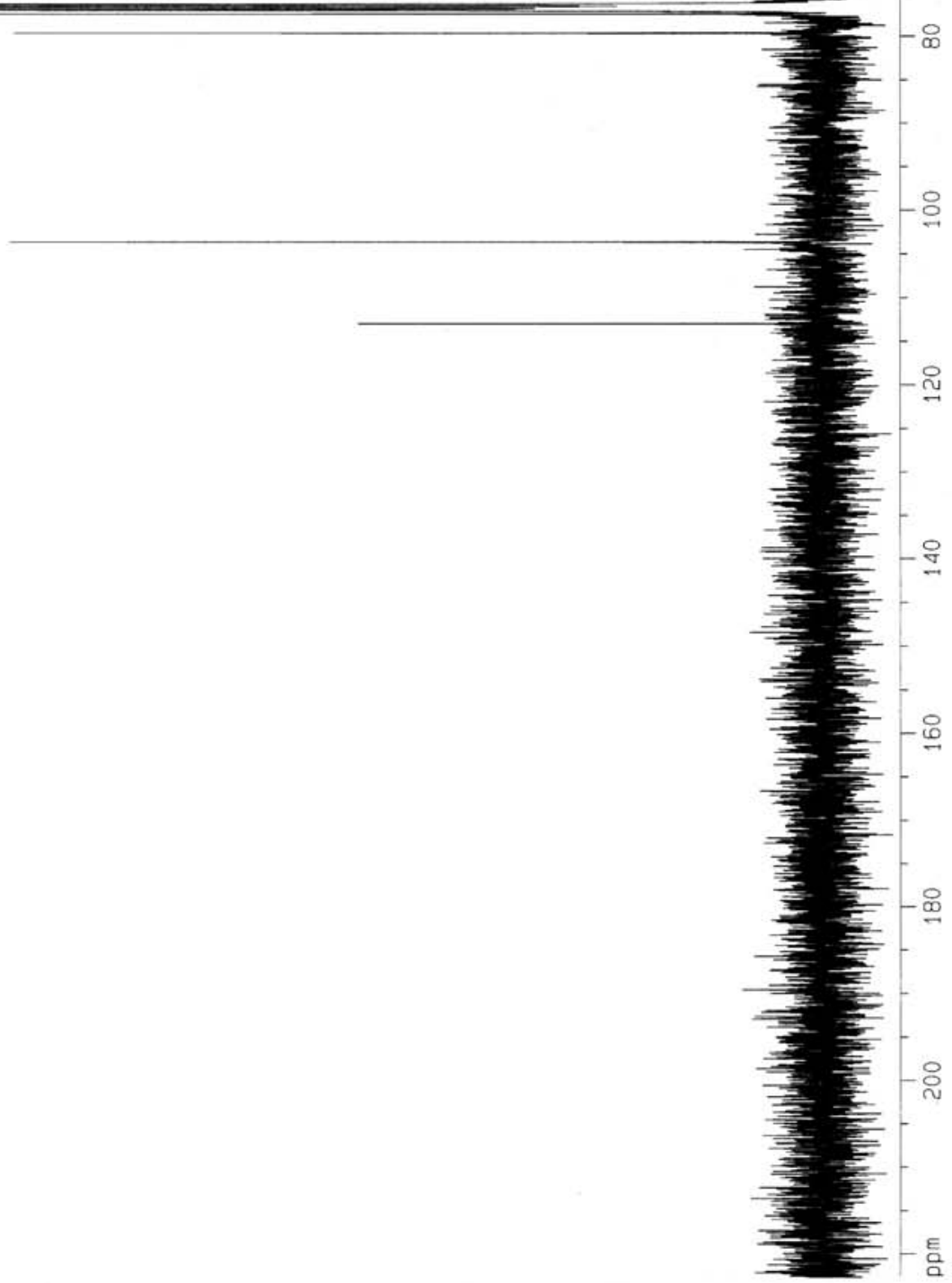




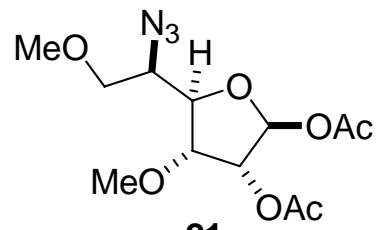

21

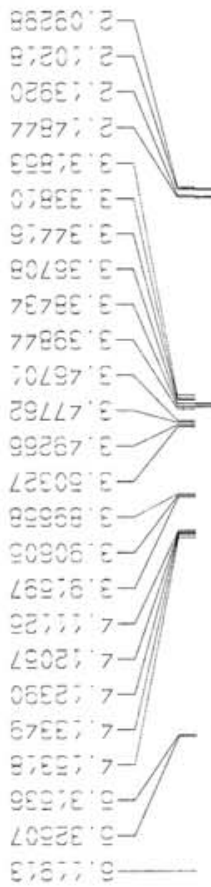

ธงวอะ $L$

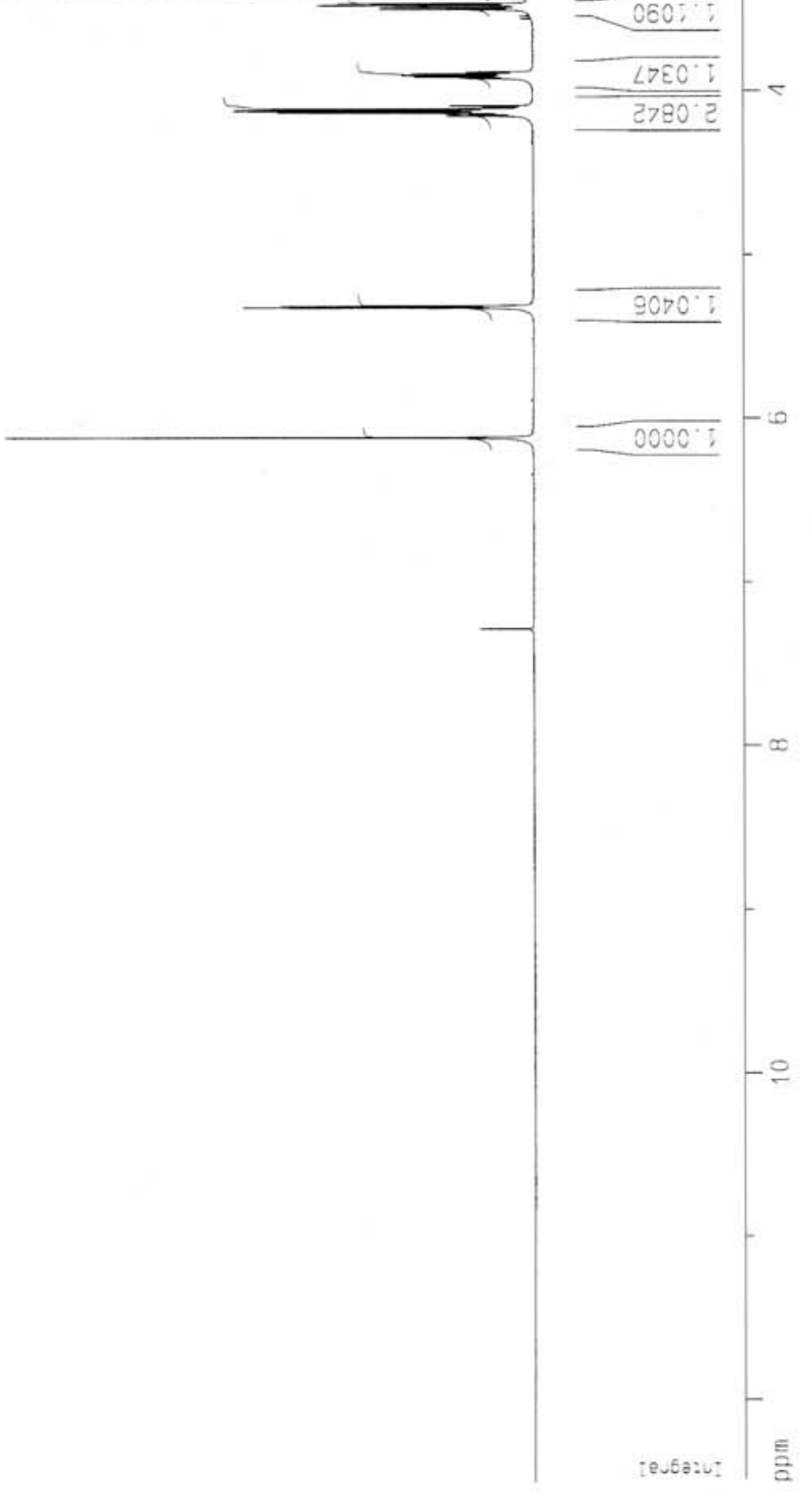




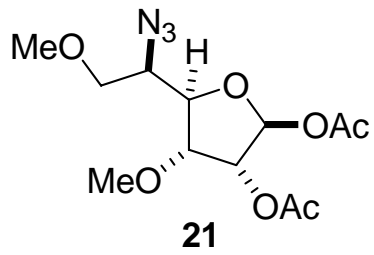

टIE. 02

ह99. 02

I2S.89

$\angle 9 L ' B 9$

6Е0.29

OE6 $0 L$

$9 \nabla 8^{\circ} \mathrm{CL}$

$\angle 8 \varepsilon^{\prime} 9 L$

$90 \angle ' 9 L$

ह20 LL

$\angle 86^{\circ} \angle L$

Eट॰ 08

IV $L ' \angle 6$

$02 L^{\prime} 891$

SटE.691- 

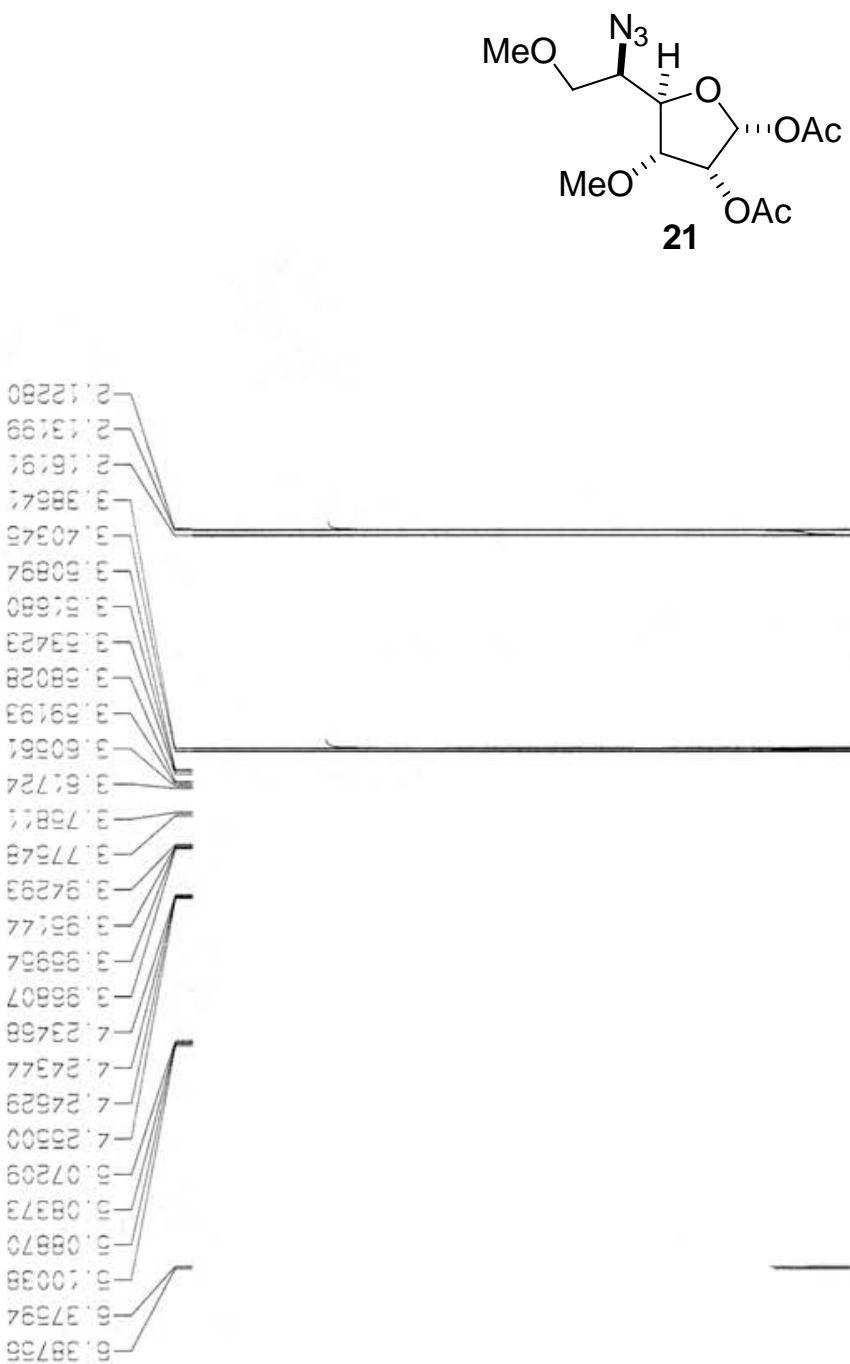

IETEE $L$

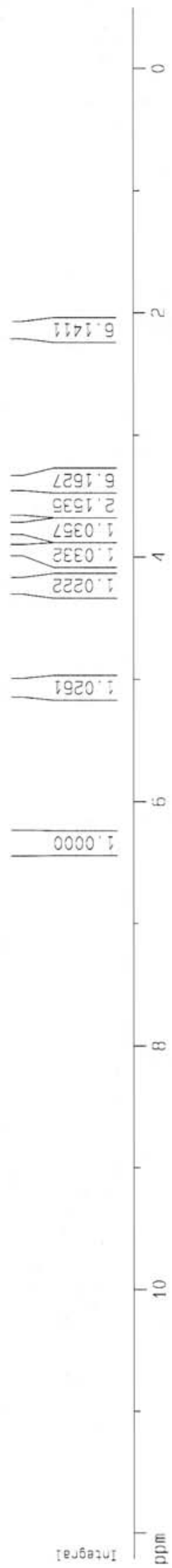




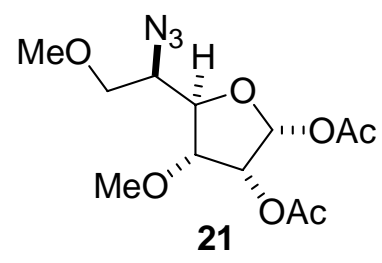

$\varepsilon I \Omega^{\circ} 02-$
$\varepsilon \varepsilon 8^{\circ} 02$

889.89
198.89

$669^{\circ} 19$

$898^{\circ} 0 L$

IST $\mathrm{L} L$

$\angle L \varepsilon^{\prime} 9 \angle$

$569^{\circ} 9 L$

$\nabla \nabla 8^{\circ} 9 L$

E10. L

$910^{\circ} \varepsilon$

टЕ૬' $\varepsilon 6$

$009691-$

$999^{\circ} 691$

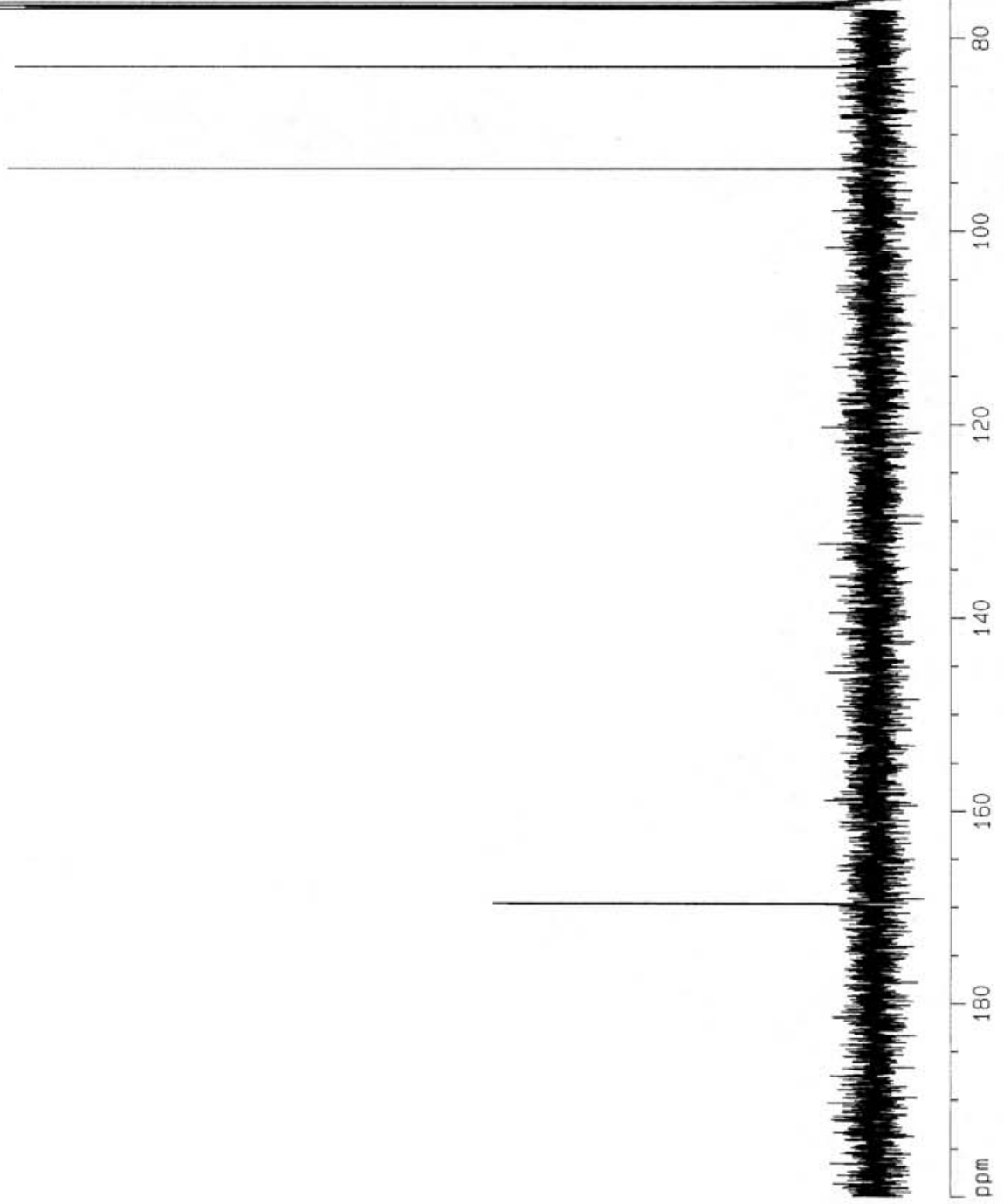



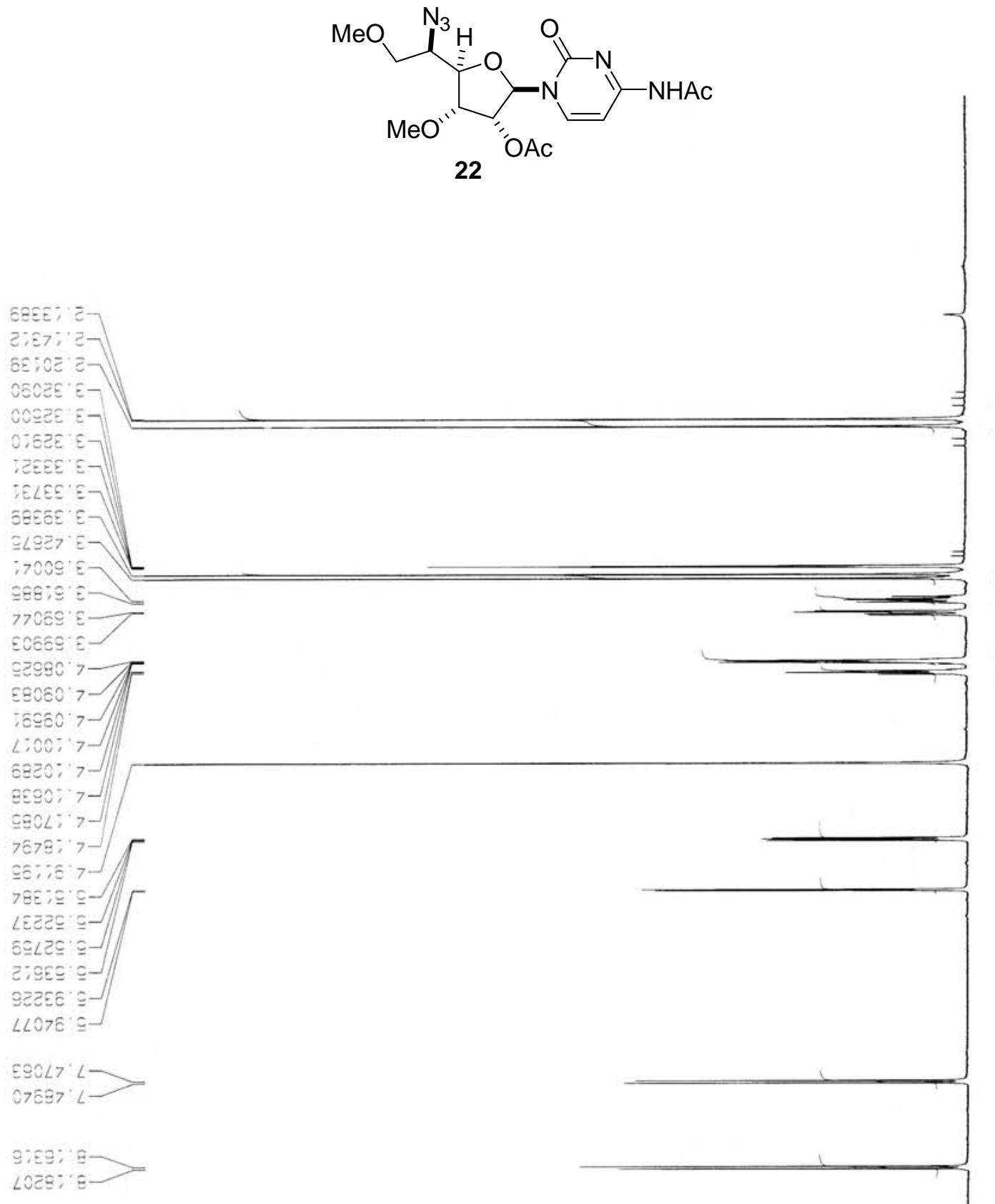

$\varepsilon 886^{\circ}$

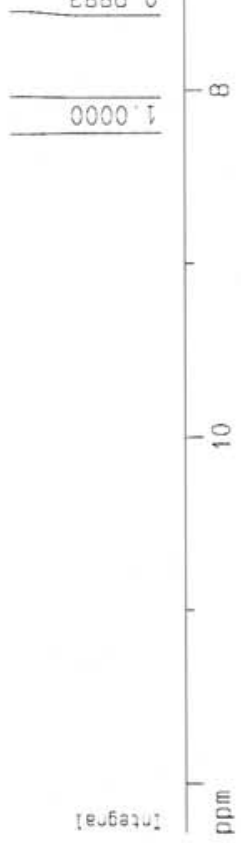




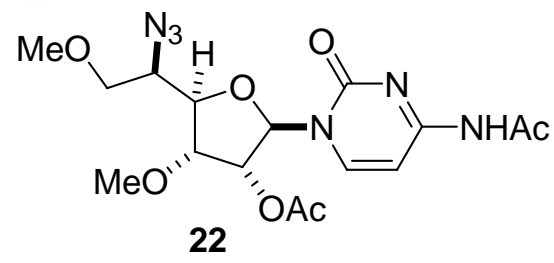

$69 L{ }^{\circ} 81$

ह6L टट

$\nabla 89^{\circ} 90$

$\angle 6 \angle{ }^{\circ} 90$

$010^{\circ} \angle D$

टटट $\angle D$

SED $\angle D$

$8 \nabla 9^{\circ} \angle D$

$198^{\circ} \angle D$

$9 \nabla G^{\circ} \angle G$

I t 19

БEट ' $\mathrm{LL}$

ODV $E L$

$9 L \angle L$

EED 08

66E 06

LEG 96
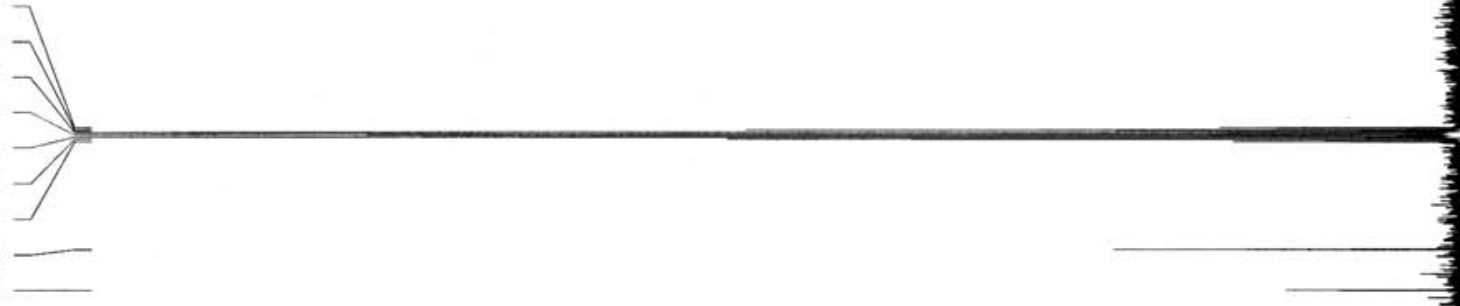
<smiles>COCC(NC(N)=O)[C@H]1O[C@@H](n2ccc(N)nc2=O)[C@H](O)[C@@H]1OC</smiles>

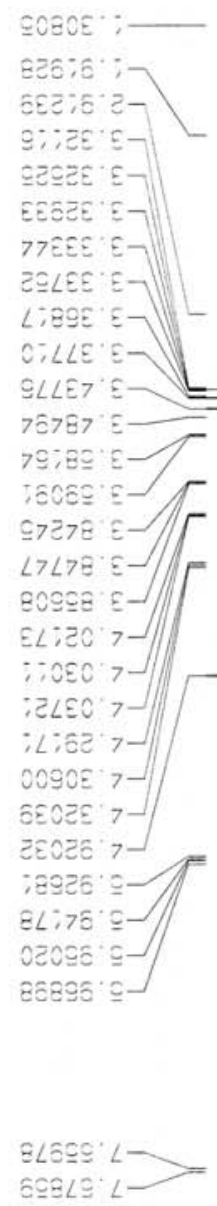

EOSLLL

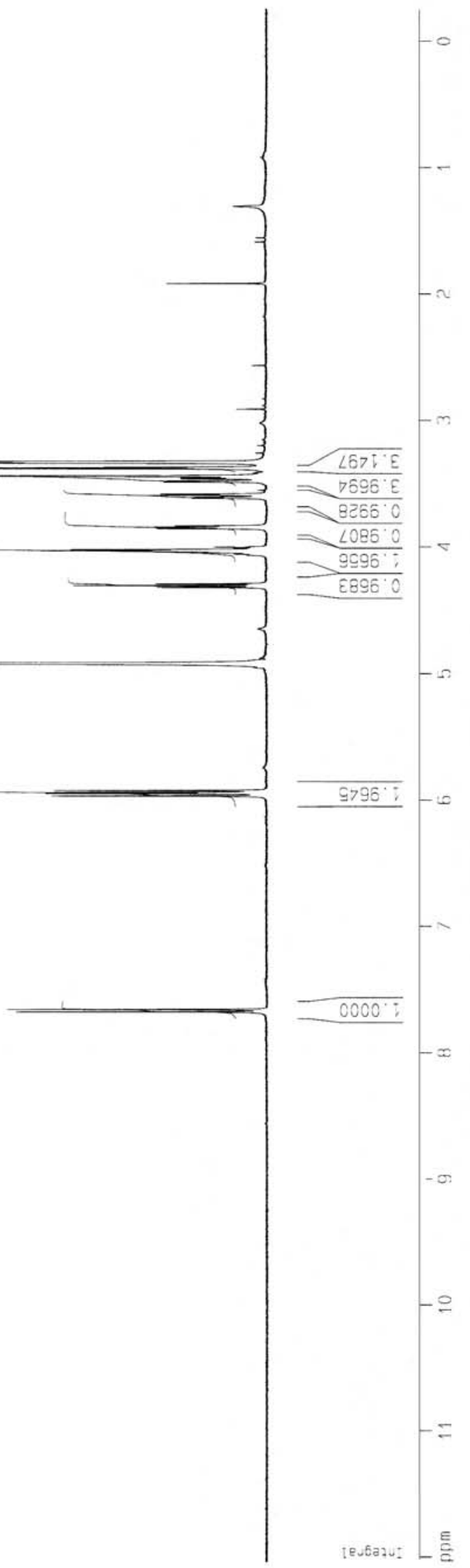




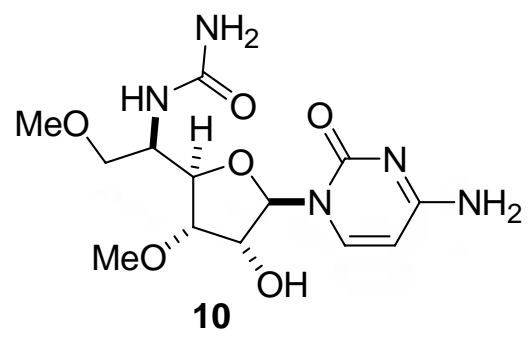




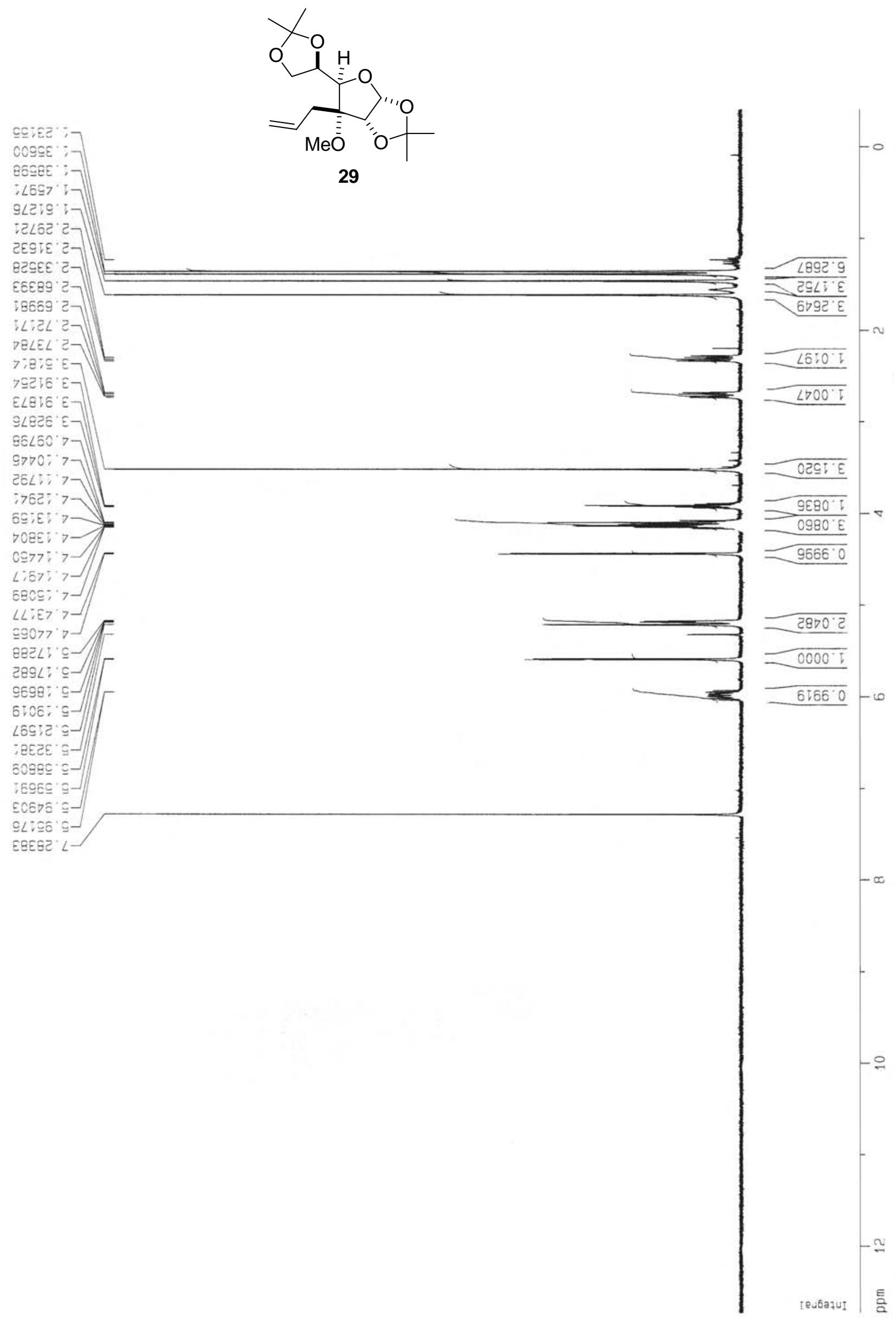


<smiles>C=CC[C@@]1(OC)[C@@H]2OC(C)(C)O[C@H]2O[C@@H]1C1COC(C)(C)O1</smiles>

29

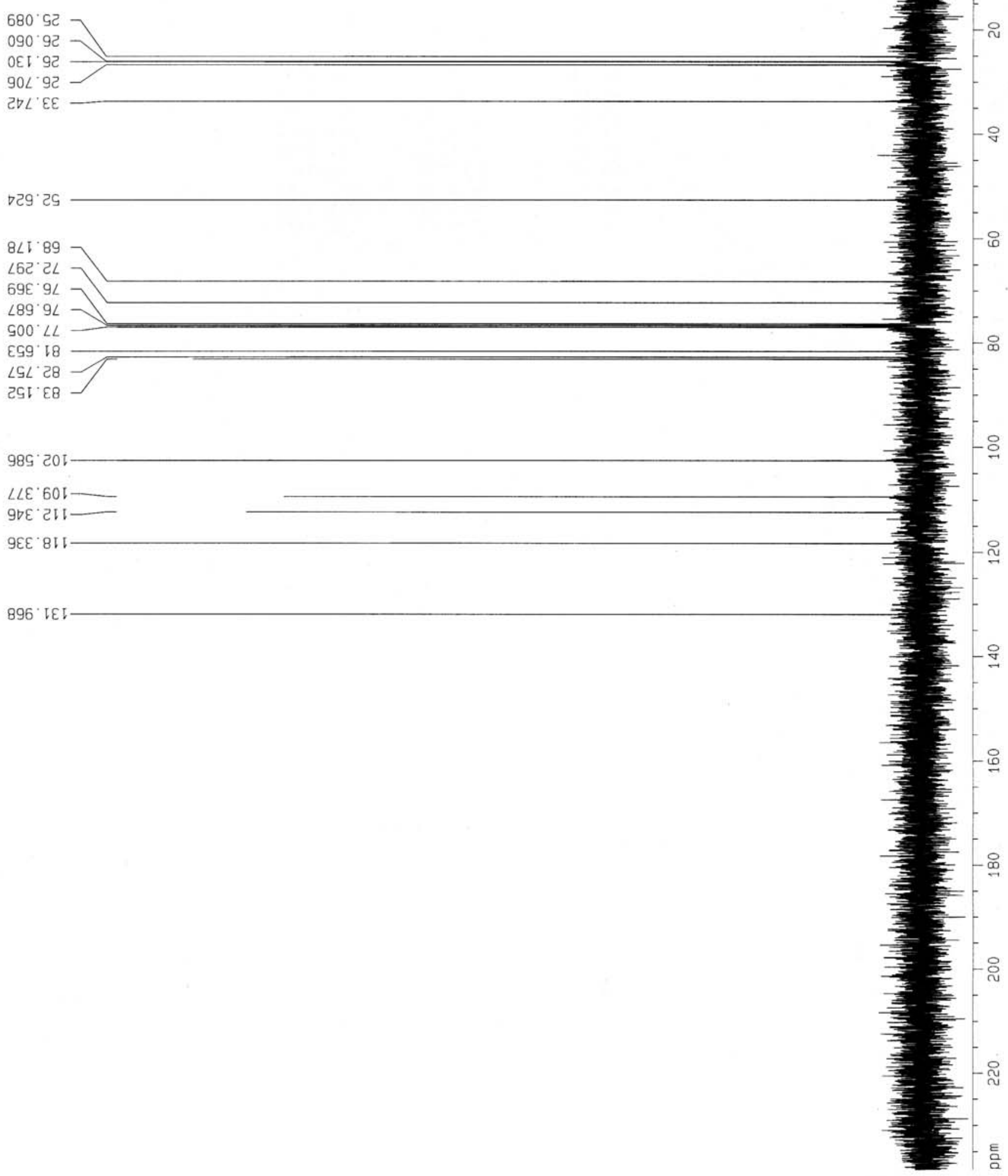

S27 


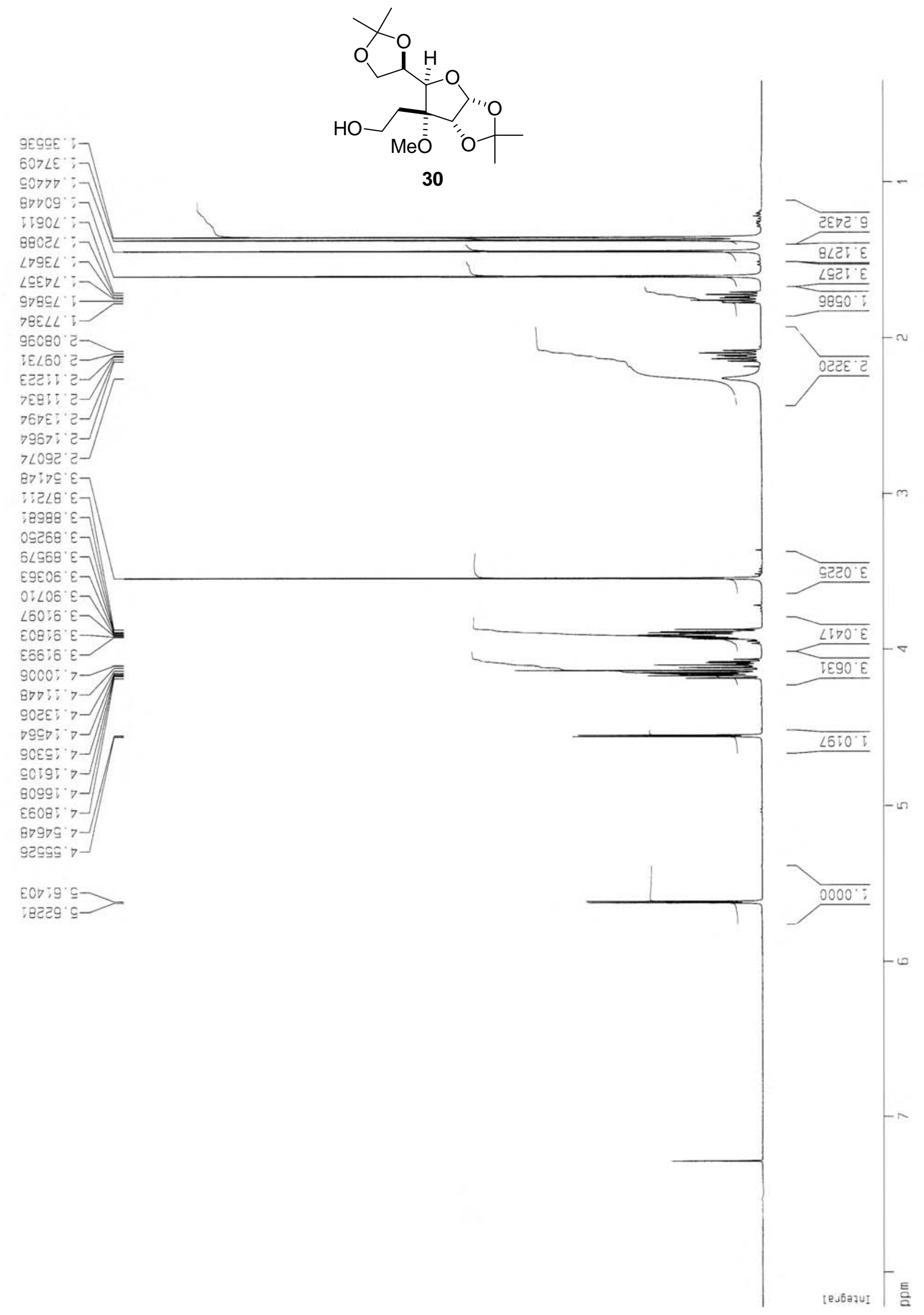


$96^{\circ} \nabla 2$
$\square \varepsilon 0.92$

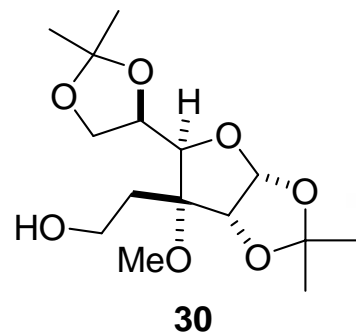

$\angle 60^{\circ} \varepsilon \mathrm{G}$

$\nabla 96^{\circ} \angle G$

$9 \angle \nabla \cdot 89$

Iटद. टL

$\varepsilon \forall \varepsilon^{\prime} 9 L$

199. 94

$8 \angle 6^{\circ} 9 \angle$

โ8ट 08

$60 \mathrm{G}^{\circ} \varepsilon 8$

$\angle 8 L \varepsilon 8$

$520_{1} \mathrm{50I}$

067.601-

199.21 -

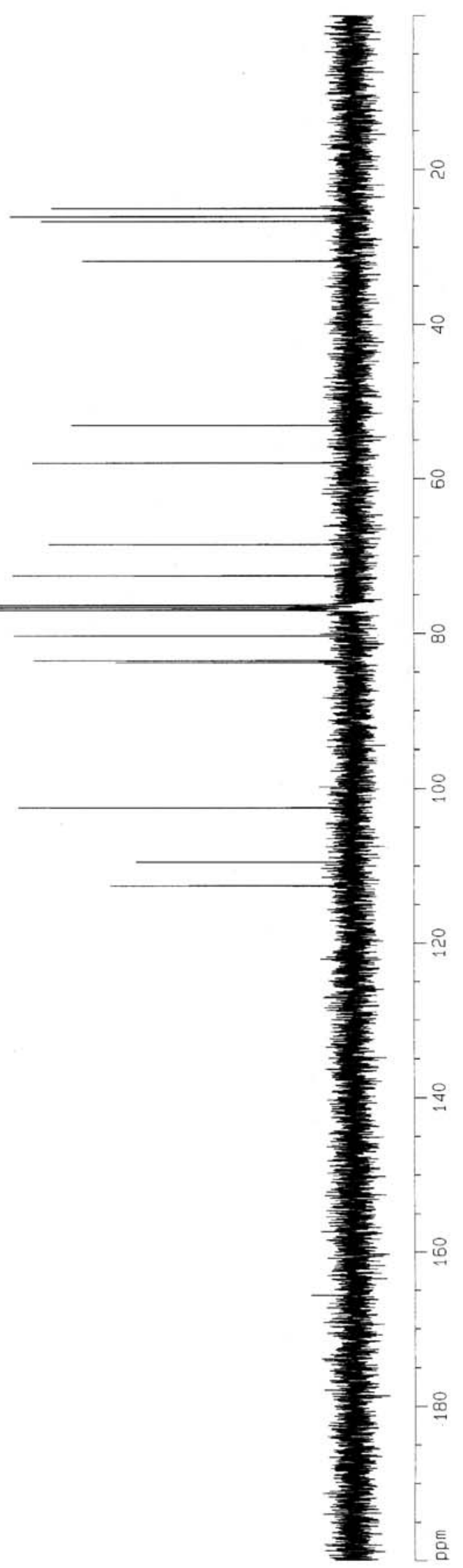



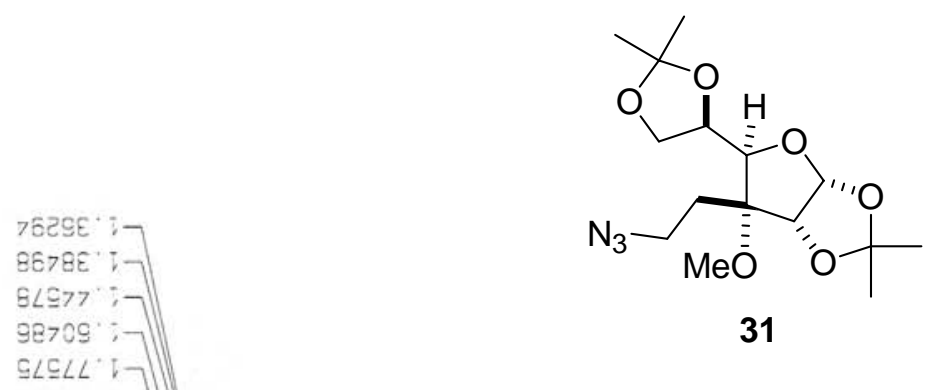

$20384^{\circ}$

टฐL6 $\angle$ '

ILZiE

จ>8:

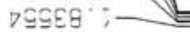

5ร390

@टन $\angle 0^{\circ} \mathrm{Z}$

$77890^{\circ}$

उ0E0

ट8 $\angle 67^{\circ}$

Co89 '

$\subseteq 8 \mathrm{C} \angle \mathrm{S}^{\circ} \varepsilon$

ट0ट89 $\varepsilon$

$86689^{\prime} \mathrm{E}$

L6969'

$68709^{\circ} \varepsilon$

$77898^{\circ}$

टLES8' $\varepsilon$

ह :688 $\varepsilon$

$89706^{\circ} \varepsilon$

6I $\angle 66$ \&

77ट:0

จEESO 7

$\triangle 9090^{\circ} 7$

〈96๐: >

9อ>९:

EEOL: $>$

ভटভ8I' $\nabla-$

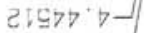

GLEGT' $7-$

EE8ว9 $\subseteq$

869E9' $\subseteq-$
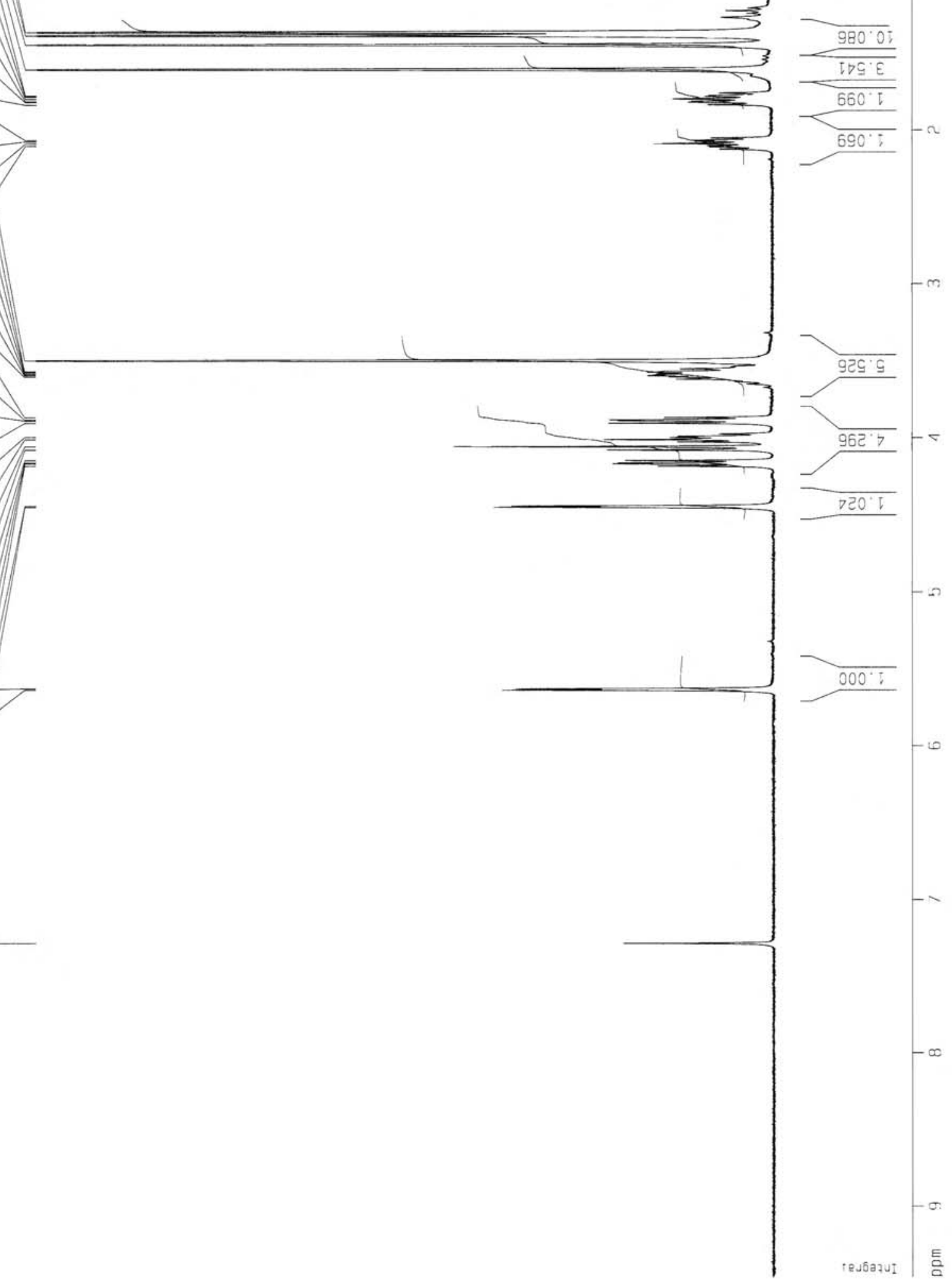
<smiles>CO[C@]1(CC[NH])C(C2COC(C)(C)O2)O[C@@H]2OC(C)(C)O[C@H]21</smiles>

31

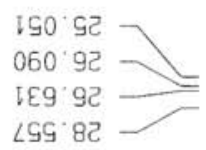

9E8. 97

ट8L' 29

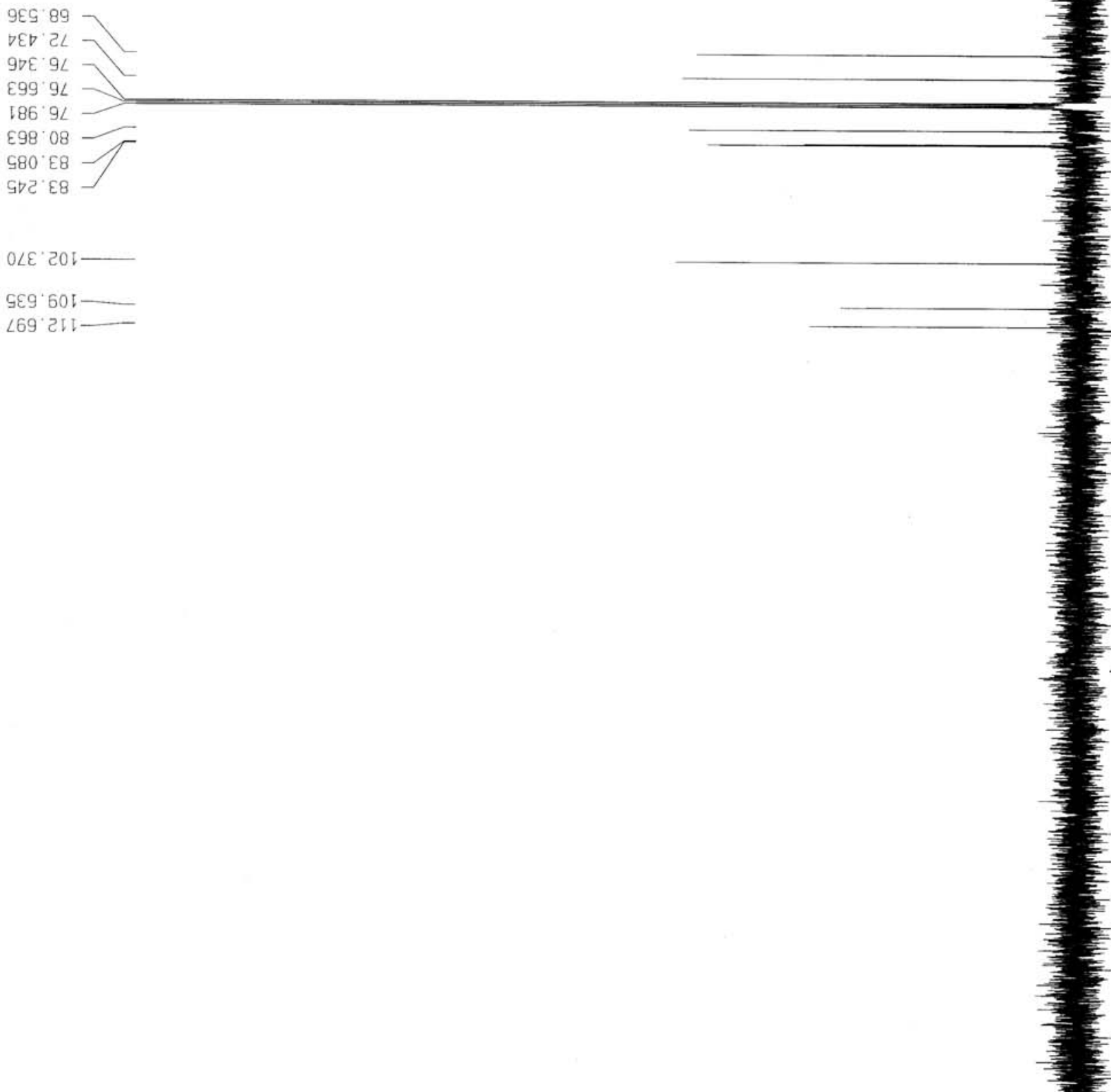

$-8$

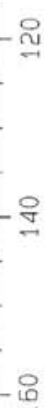

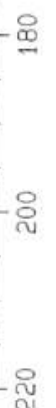

틈

S31 


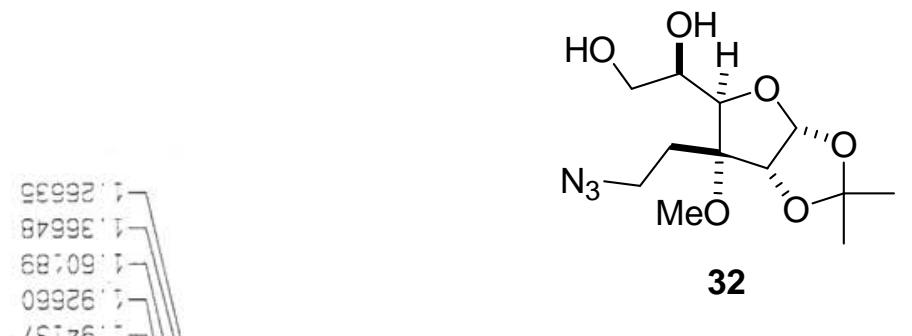

$\angle E i 76^{\prime}$;

घ6876

हE796

$\angle O Z E 0^{\circ}$

ESS००

$\operatorname{cSD} 200^{\circ} \bar{c}$

$\angle 90 \angle 0^{\circ} \mathrm{Z}=$

टLCEO

$\varepsilon 9050^{\circ} \mathrm{C}$

ฐ7767 ह

ienge

ट马S $\angle \mathcal{G}^{\prime} \mathrm{E}$

E7EอG' $\varepsilon$

6ธยธธ '

๑จธธต' $\mathrm{冫}$

8:979

$\nabla C \angle 9^{\circ} \varepsilon$

c⿻E89

टह७69 $\varepsilon$

EOELL'E-

gIEB $\angle$ ' $\varepsilon-$

$77^{\prime} 6 L$ ' $E-$

$7686 \angle \varepsilon-\int$

$9 \Xi \angle 0 \theta^{\prime} \varepsilon$

Gट928

$907: 0 \div$

ट0950.

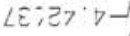

9EOE7 7

$067 \angle 9^{\prime} 9$

685g9.
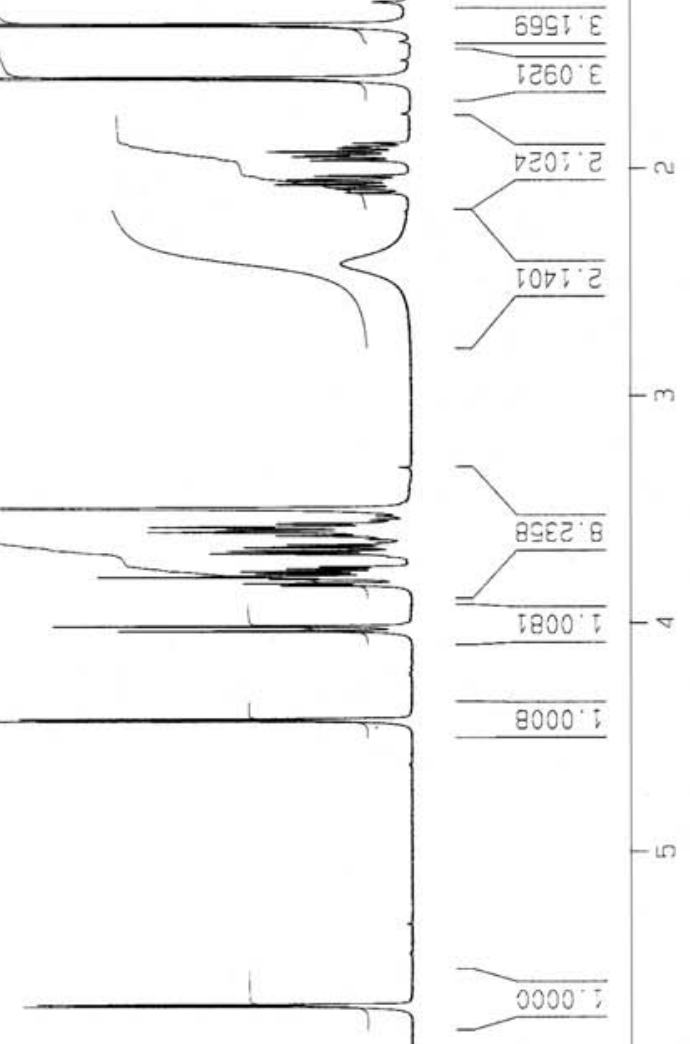

EgEge $L$

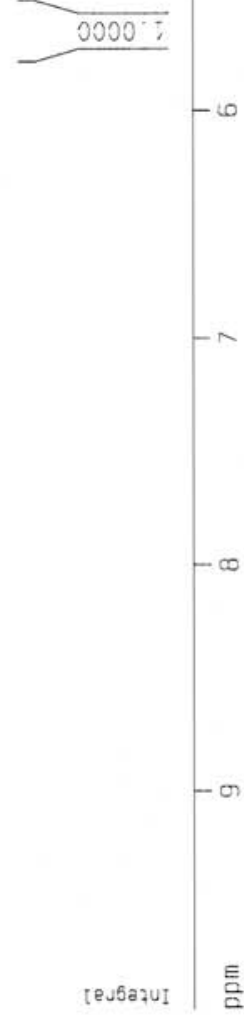




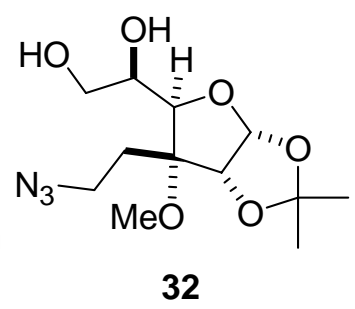

I0I 92

$8 \angle \varepsilon^{\circ} 92$

$610.62-$

696. 95

926.29 -

$\nabla 6{ }^{\circ} \nabla 9-$

$98 \varepsilon^{\circ} 69$

टSE' 94

$\angle 86^{\circ} 9 \angle$

OS6 $\angle L$

$\varepsilon$ हट 28

$88 L^{\circ} \mathrm{E8}-$

$\angle O^{\circ}{ }^{\circ} \mathrm{OL}-$

9L8. 51

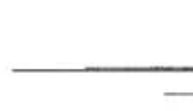




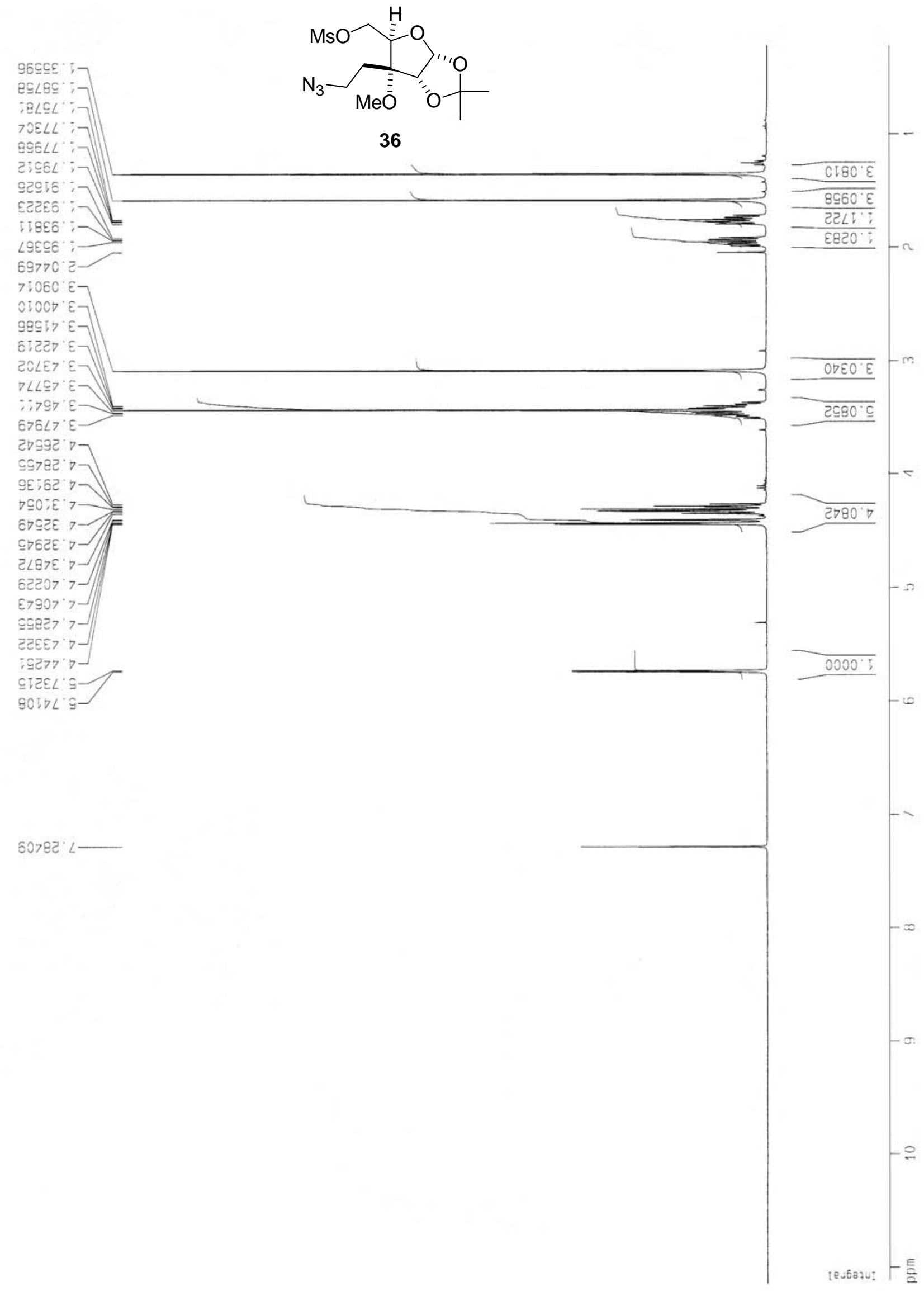




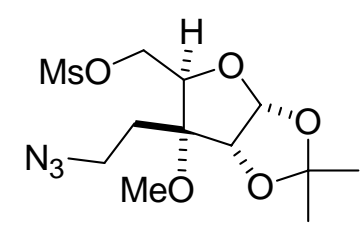

36

910.92

68ह 92

6ट. 82

$90 \mathrm{~S}^{\circ} \angle E$

$809^{\circ} 96$

999 25

टOE $\angle 9$

$100 \cdot 9 L$
$6 L / 9 L$

Оट6. $9 L$

$\angle E O^{\circ} \angle L$

$G \angle 8^{\circ} \angle L$

828 เ8

$\checkmark D^{\circ} 28$

6อป ย०I

$\angle E \sigma^{\circ} \mathrm{Cn}$ 


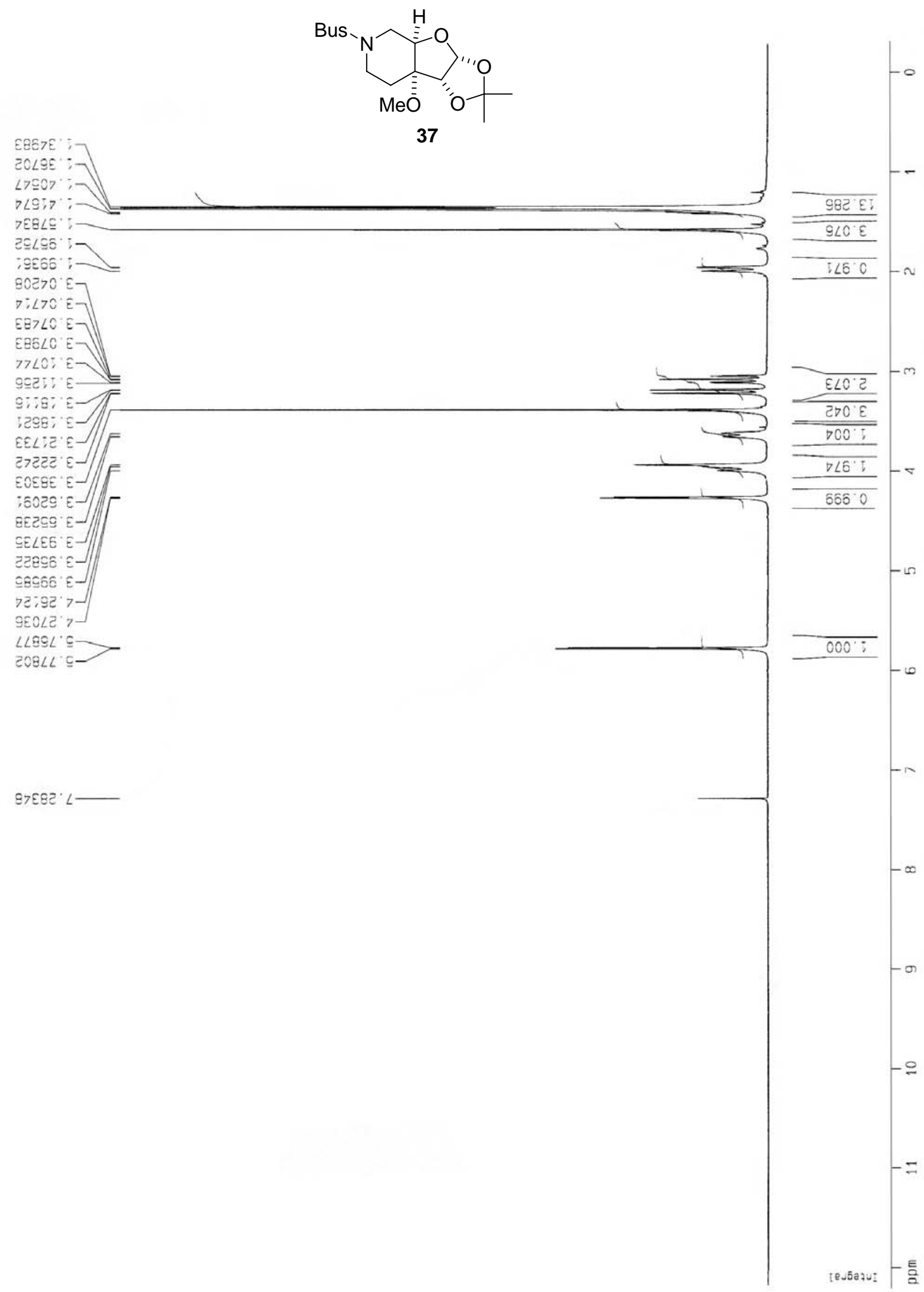



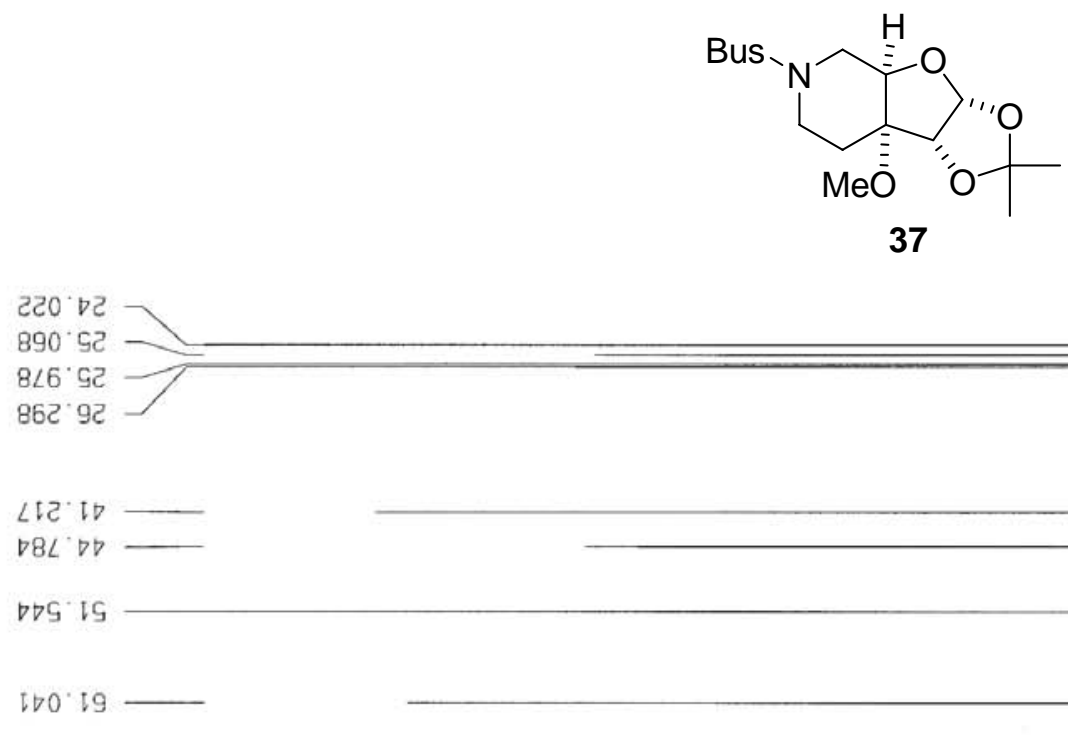

SEI $D L$
EBE' $9 L$
TOL $9 L$
$610^{\circ} \angle L$
IV' $8 L$
$\angle L E^{\circ} 08$

$\nabla \varepsilon 8^{\prime} \varepsilon 0 \mathrm{~T}$

$\angle \varepsilon 9211-$ 

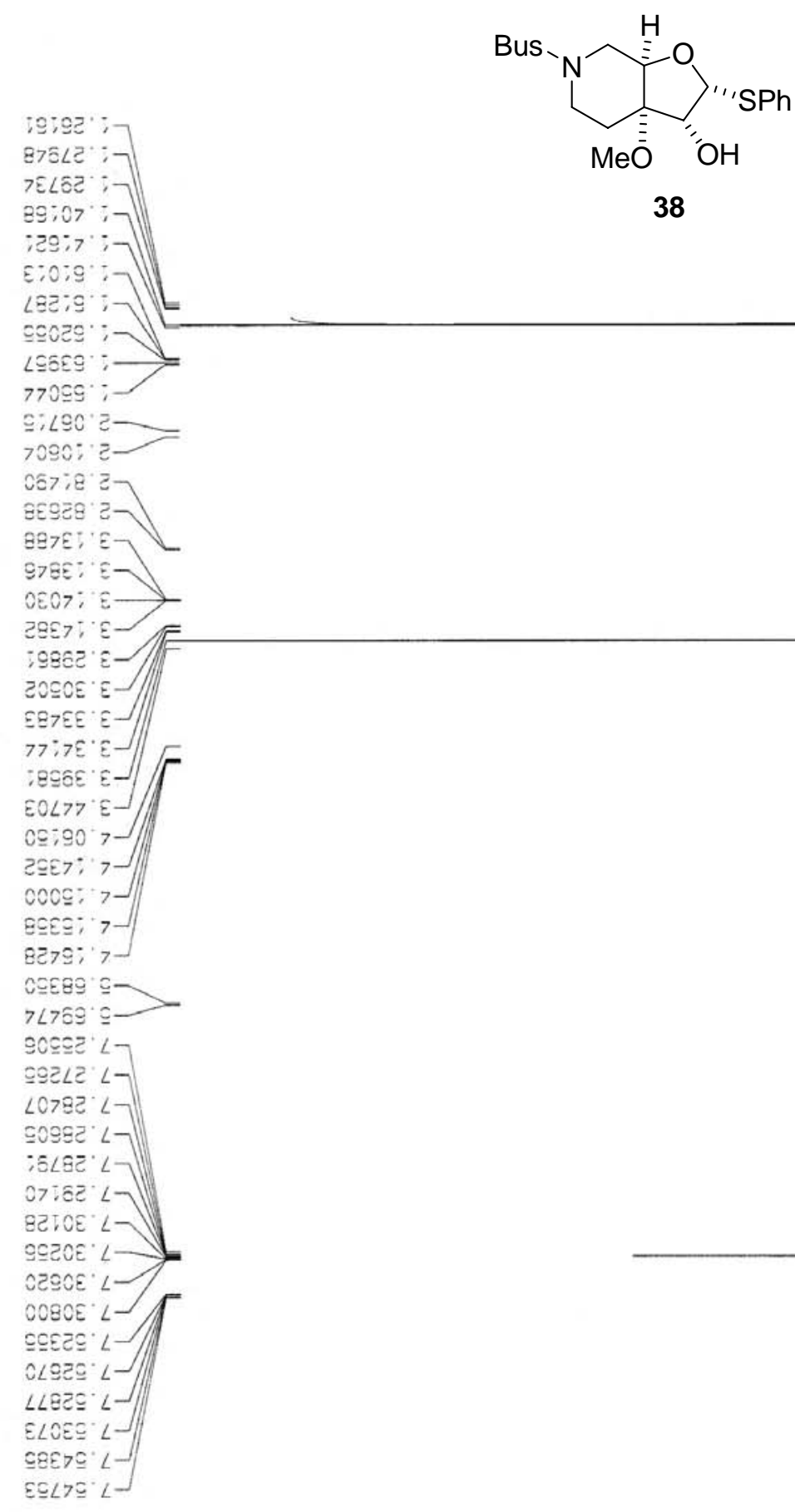


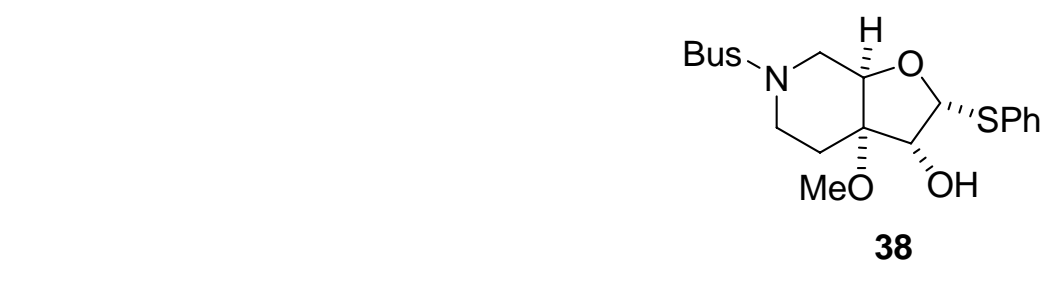

ตा0 $\nabla 2$

५8. $92=$

$\angle E L$ IV
29D $9 D-$
99D IS

$961 \cdot 19-$

$\square \angle E^{\prime} \varepsilon L$

$\varepsilon O I^{\circ} \nabla L \longrightarrow$

$\angle 89^{\circ} 9 L$
900.

E9E. $8 L$

६૬६' ट6

$199.921-$

9VG 82 I

EES OE : -

EII GE-
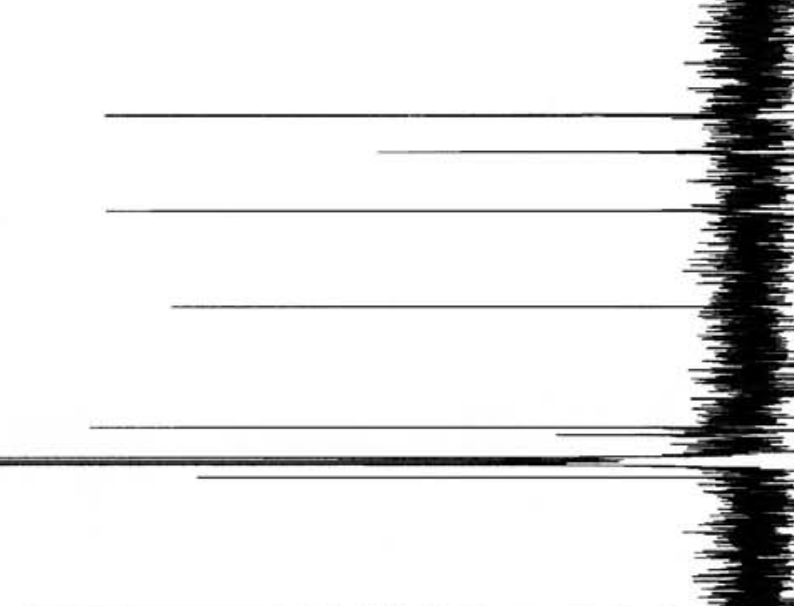

$-\infty$

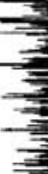




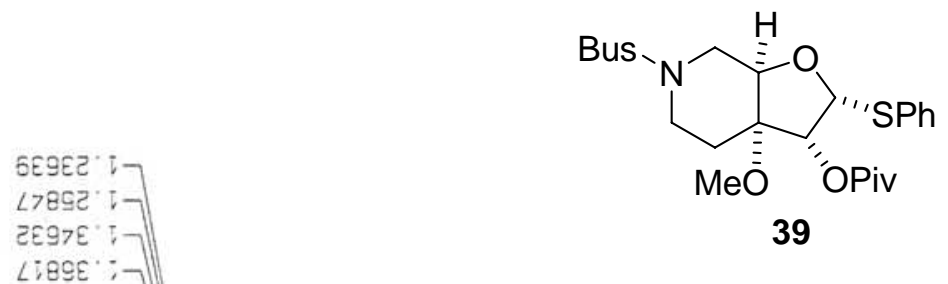

LLGES :

iegg : :

96: $\angle 9^{\prime} ;$

टBฐ $\angle \subseteq$

$86 \angle 69^{\prime}:$

i9809

is060'

$88 \angle 5,5-1$

$\angle$ CETO

TLEDO

〔

$\angle I G \angle 0^{\circ}$

$99080^{\circ} \mathrm{g}$

ย泬:

LEgEट $E-$

घटEमट ह-

$897 \angle Z E-\mid$

$9 \subseteq 6 \angle Z^{\prime} \varepsilon-$

E079 ' $\varepsilon$

$97969 \cdot$

GE9:0 $7-$

$96290^{\circ} \circ$

Eg960 $7-$

E8ा

ЄटЕट๐

89LiL $\cong$

$6062 L 9$

ยฐフフट

$\angle 9: 9 C^{\prime} \angle$

$80892<-$

$\nabla 9 \operatorname{tgc} L$

7 7692

$\triangle 2782^{\circ}$

EE $\angle B C$ C $\angle$

Сฐ६6อ ' $\angle$

$\triangle 己>O E$ : $\angle-$

$\triangle G 60 E^{\circ}<-$

$\angle 9880^{\circ} \angle-$

67 泊 $\angle$

gट809 $\mathrm{L}$

OIsts L-

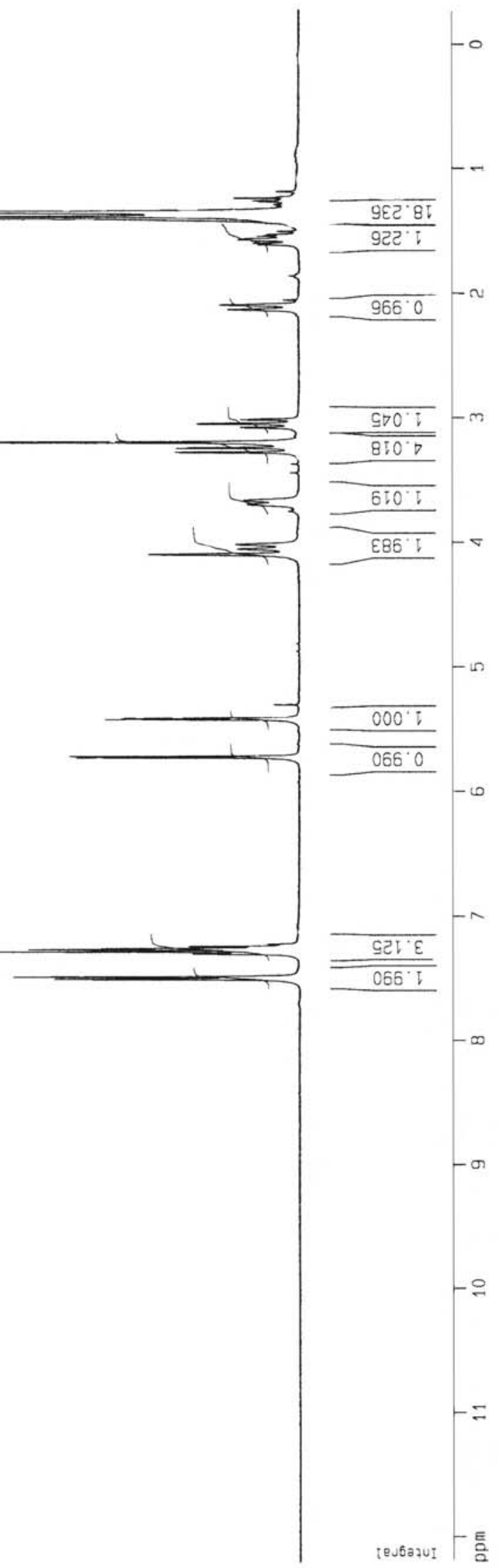



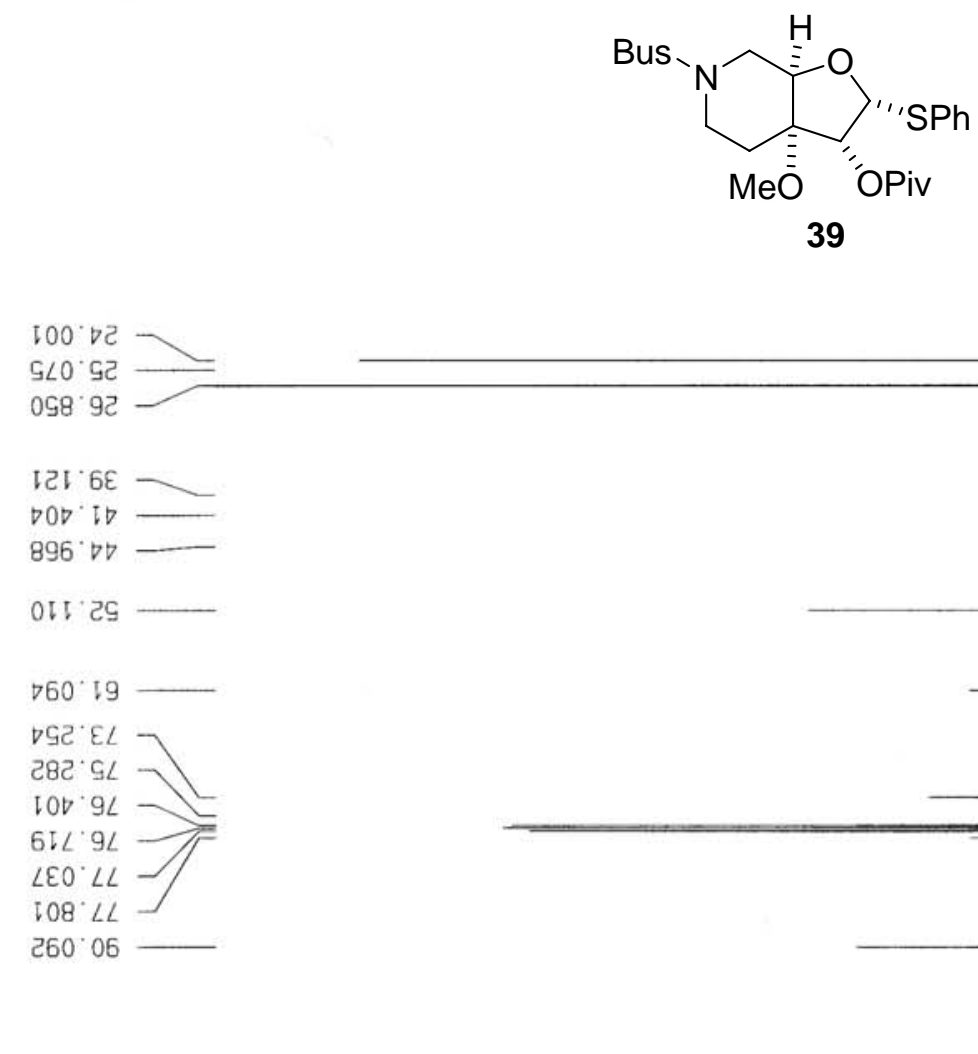

$098^{\circ}$ 9ट

$\angle 99^{\circ} 8 \mathrm{C}^{\circ} \longrightarrow$

ट09 OE

¿SL $\nabla E T-$

$D S S^{\circ} 9 \angle L$

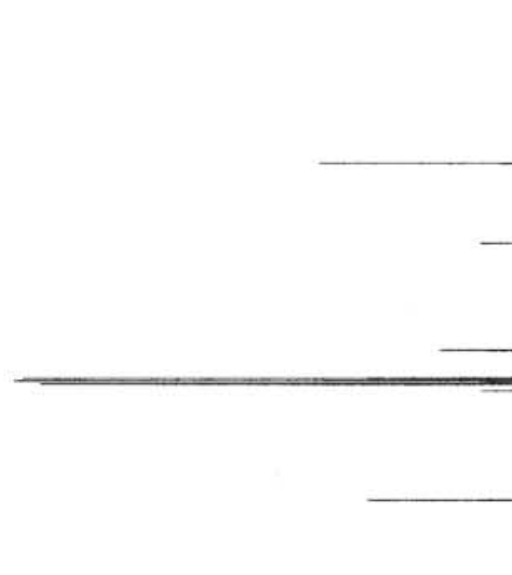




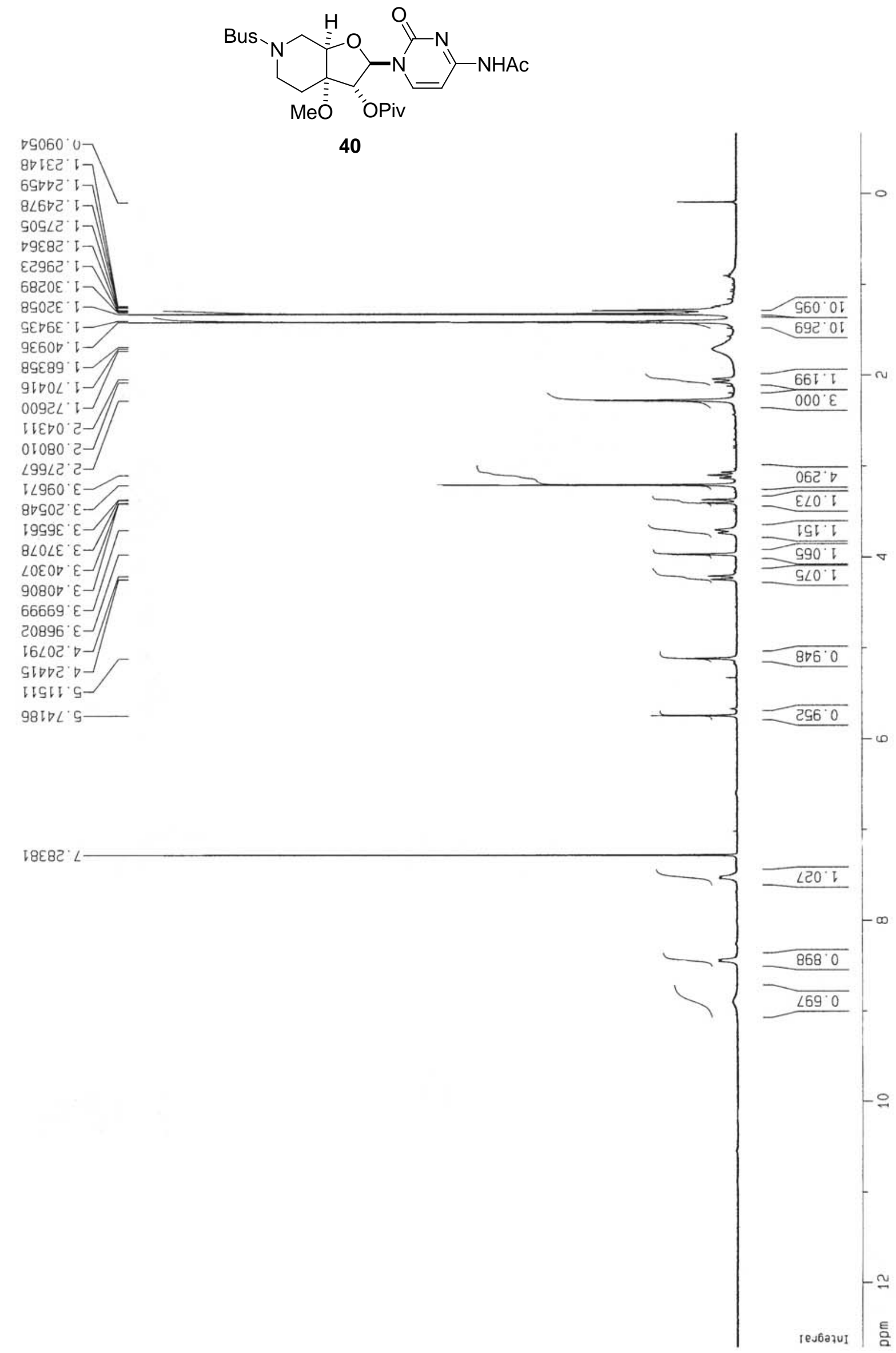




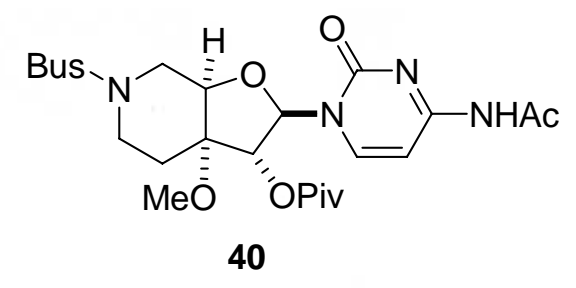

$\varepsilon \angle 0^{\circ} \nabla C$
$\nabla 99^{\circ} \nabla C$
$182^{\circ} 92$
$889^{\circ} 92$

$0 \angle 019-$

$908^{\circ} \mathrm{SL}$

$0 \angle E^{\prime} 9 \angle$

EOG $9 /$

$\angle 89^{\circ} 9 \angle$

$688^{\circ} 9$

$\mathrm{S} 00^{\circ} \mathrm{LL}$

ESD V6

$699^{\circ} 96$

40

I8G EDI

9EE $\nabla G I-$

$188^{\circ} 291-$

$908^{\circ} 0 \angle \mathrm{V}$

$088^{\circ} 9 \angle 1-$

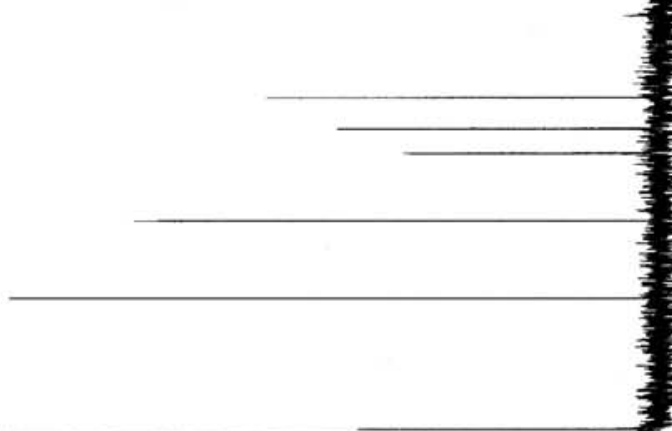

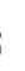


$\angle \angle 9 ;: 0$

96972 :

;60 $6 \mathrm{C}^{\prime} ;$

SLOEट

टग96ट

LiEOE' '

洒 708

$07660^{\circ}$

बह९६:

$\angle O S \angle 9^{\circ}$

GLE:;

$6897 i$

海 LI'E

вटЕटह

EOS6E :

$\angle \supset E 97$

GIVIL'

$\angle E O T L$

$88 \nabla \subseteq \angle \cdot E$

$9 \angle 080^{\circ} 7$

จ290: 7

$\angle O \angle O)^{\circ}>$

990::

LLGE:

ट $\angle 787^{\circ}$

हणटटट

gEI96 $7-$

26960 9

टट:0L

66Е7;

हก:อง 9

$08 \angle 98 \angle$

$: 9 \angle \angle \theta^{\circ} \angle \longrightarrow$

)<smiles>CO[C@]12CCN(C(N)=O)C[C@H]1O[C@@H](n1ccc(N)nc1=O)[C@H]2O</smiles>

11

\footnotetext{
列
}
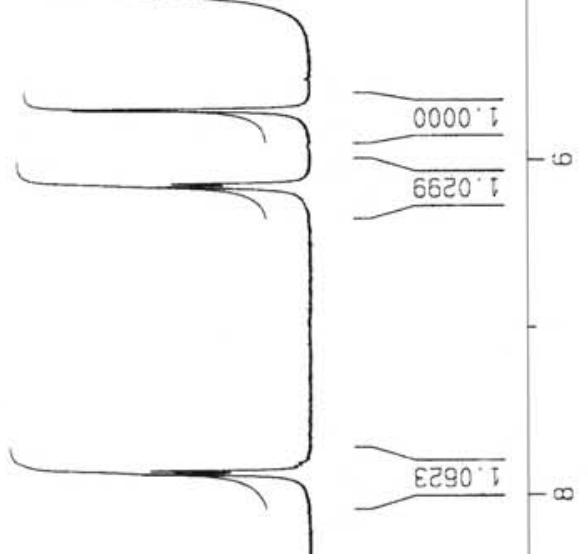

으

$-\cong$

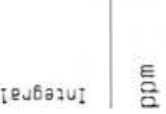



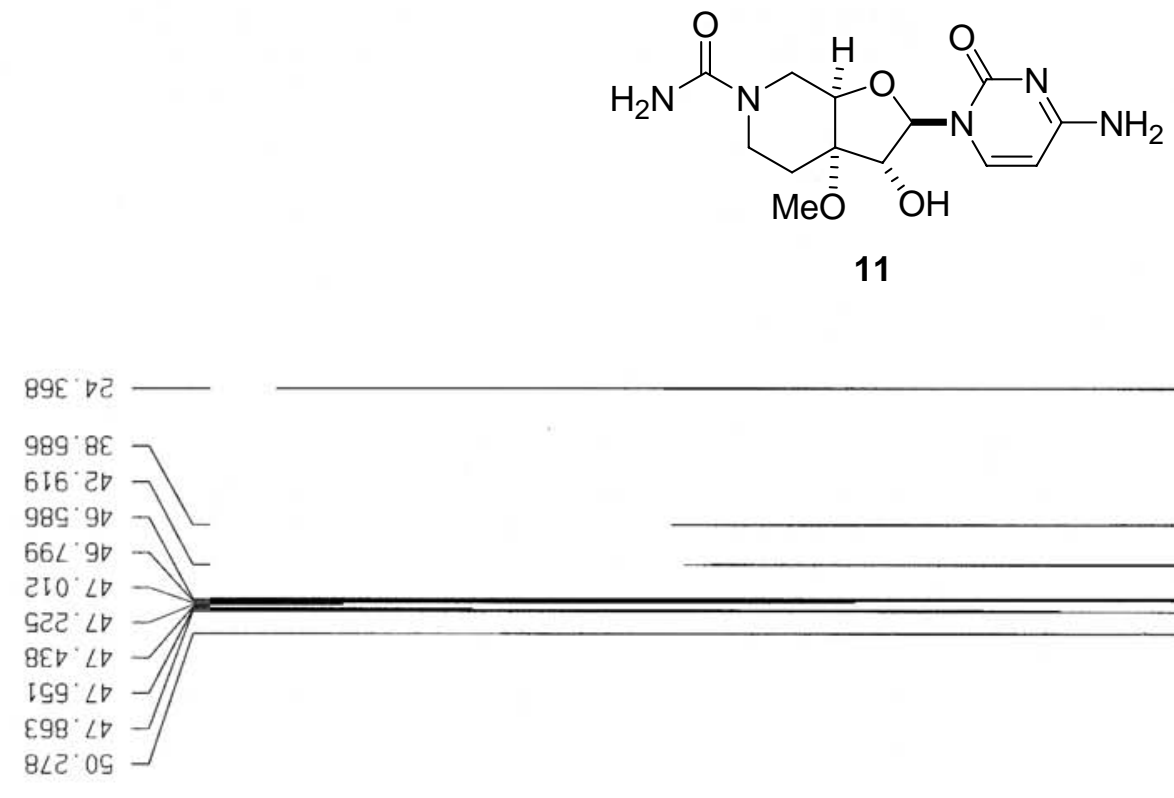

$\nabla \varepsilon S^{\prime} \mathrm{SL}$

IEE $\angle L$

ITO' $8 L$

$60 \varepsilon \cdot 26=$
$\angle 9{ }^{\circ} \cdot \mathcal{\prime} 6$

$688 \cdot 271-$

OEI $\angle D I-$

LOS. $691 \longrightarrow$

$979.691-$

11 


\section{Crystal structure determination and refinement of compound 18 and ORTEP}

\section{representation (see also accompanying cif file)}

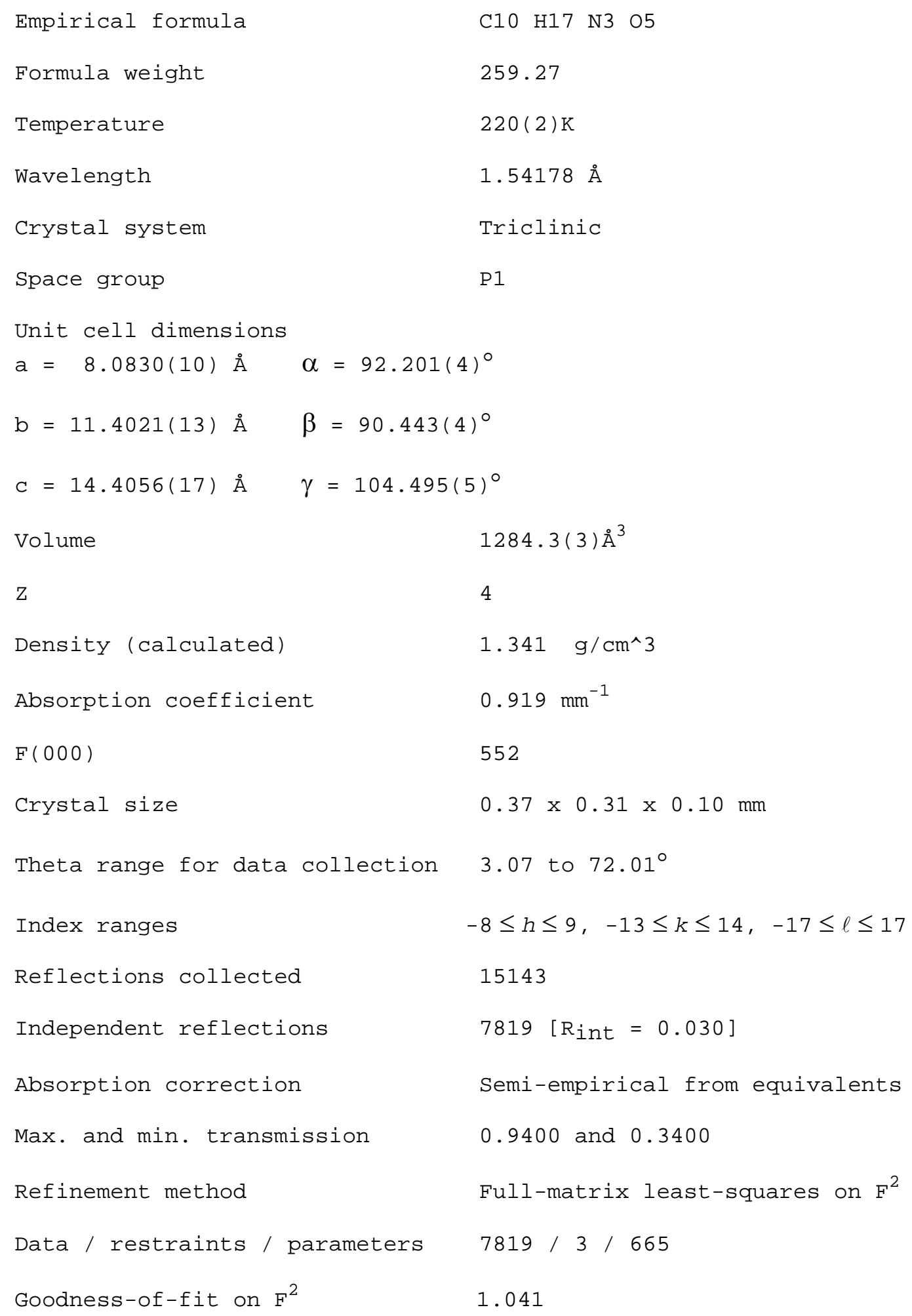




$\begin{array}{ll}\text { Final } R \text { indices [I>2sigma(I)] } & R_{1}=0.0482, W R_{2}=0.1284 \\ R \text { indices (all data) } & R_{1}=0.0506, W R_{2}=0.1318 \\ \text { Absolute structure parameter } & 0.08(11) \\ \text { Largest diff. peak and hole } & 0.313 \text { and }-0.232 \mathrm{e} / \AA^{3}\end{array}$

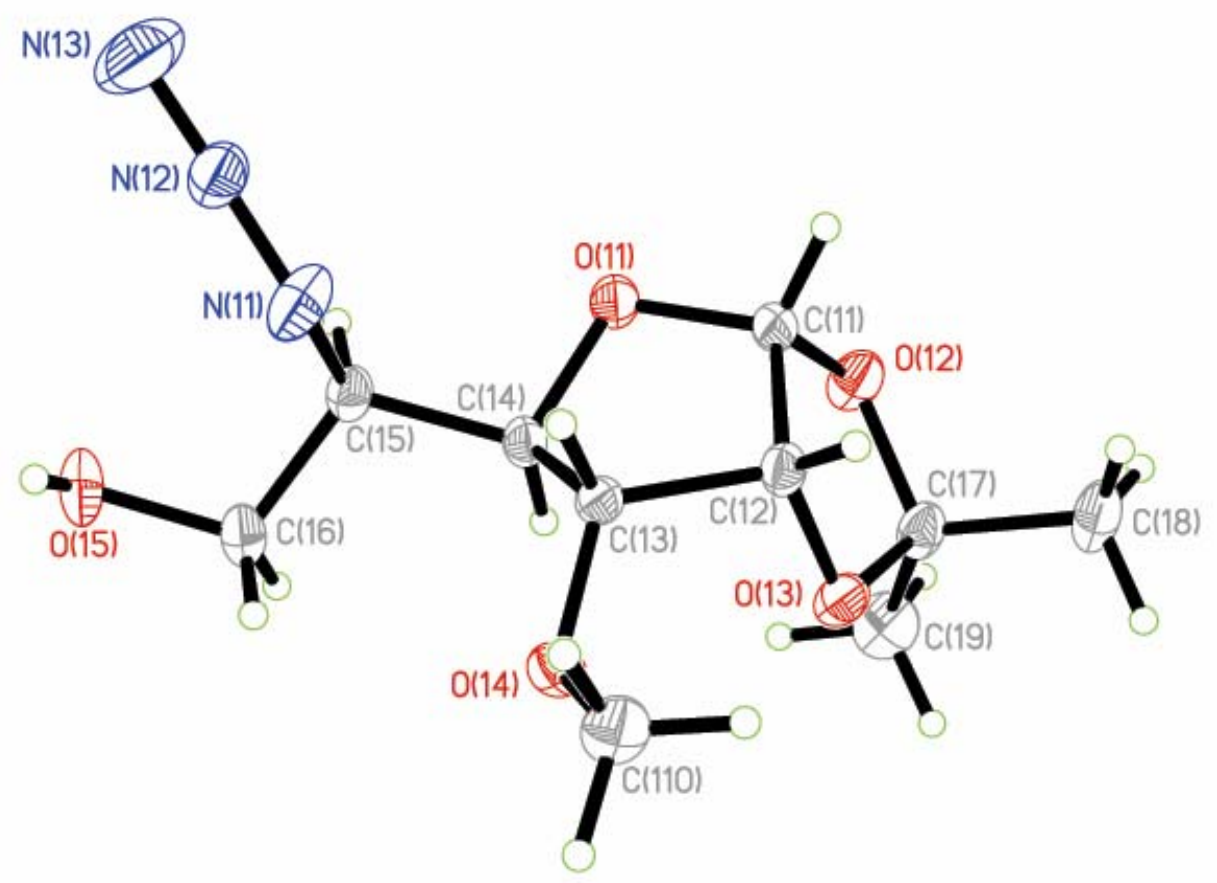




\section{Crystal structure determination and refinement of compound 44 (see manuscript for ORTEP representation and also accompanying cif file)}

Crystals suitable for diffraction were obtained from a mixture of iso-propanol and water. Diffraction data were collected at $100 \mathrm{~K}$ with a Bruker AXS SMART $6000 \mathrm{CCD}$ detector on a three-circle platform goniometer with graphite monochromatized $\mathrm{Cu}(\mathrm{K} \alpha)$ radiation from a sealed tube generator. A semi-empirical absorption correction was applied, based on the intensities of symmetry-related reflections measured at different angular settings (Sheldrick, G.M. SADABS, University of Göttingen, Göttingen, Germany). The structure was solved and refined on $\mathrm{F}^{2}$ with the SHELXTL suite of programs (Sheldrick, G.M. SHELXTL, version 6.12, Bruker AXS inc. Madison, Wisconsin, USA). Compound 44 crystallizes with 0.75 equivalents of water and 0.5 equivalents of iso-propanol with two independent molecules in the asymmetric unit. Non-hydrogen atoms were refined with anisotropic displacement parameters. Hydrogen atoms were located in a DF map and refined in idealized positions using a riding model.

Final data: $\mathrm{C}_{14} \mathrm{H}_{21} \mathrm{~N}_{5} \mathrm{O}_{5} \cdot 0.75 \mathrm{H}_{2} \mathrm{O} \cdot 0.5 \mathrm{C}_{3} \mathrm{H}_{8} \mathrm{O}, M_{\mathrm{r}}=382.92$, orthorhombic, space group $P 22_{1} 2_{1}$ (No. 19) with $a=11.230(2), b=13.374(3), c=25.007(5) \AA, V=3755.8(13) \AA^{3}$, $Z=8, D_{\mathrm{c}}=1.354 \mathrm{~g} \cdot \mathrm{cm}^{-3}, 53116$ reflections measured, 6635 independent, $3.53^{\circ}<\theta<$ $66.69^{\circ}, \mathrm{T}=100(2) \mathrm{K}, 494$ parameters, no restraints, $\mathrm{R}_{1}=0.0445, w \mathrm{R}_{2}=0.0974$ for 6024 reflections with $I>2 \sigma(I), \mathrm{R}_{1}=0.0516, w \mathrm{R}_{2}=0.1013$ for all 6635 data, $\mathrm{GoF}=1.073$, res. el.dens. $=+0.41 /-0.29 \mathrm{e} \cdot \AA^{3}$. 
Crystallographic data (excluding structure factors) for $\mathbf{4 4}$ have been deposited with the Cambridge Crystallographic Data Centre which has the deposition number CCDC 638759. 


\section{Tables for energies and HF calculations, for 7, 42, 43}

Conformations

Egaz (a.u) RHF/3-21G*

Cytosinyl-N-malayamycin A (7)

$\begin{array}{ll}\text { Chair } & -1217.9841394 \\ \text { Twist boat } & -1217.9839737 \\ \text { Boat } & -1217.9748085\end{array}$

$\underline{e p i-\mathrm{C}_{6}}$-methoxy- $N$-malayamycin (42)

Chair $\quad-1217.9807958$

Twist boat $\quad-1217.9789014$

Boat $\quad-1217.9646602$

C $_{6}$-fluoro- $N$-malayamycin (43)

Twist boat $\quad-1203.0556427$

Chair $\quad-1203.0520118$

Boat $\quad-1203.0452409$

a single point calculation on the fixed optimized conformation RHF 3-21g* using Spartan.

\begin{tabular}{|c|c|c|c|c|c|c|c|}
\hline EADER & \multirow{2}{*}{\multicolumn{7}{|c|}{$\begin{array}{l}\text { Cytosinyl-N-malayamycin A ( } 7 \text { ) boat conformation } \\
\text { Spartan exported Wed oct } 26 \text { 19:07:18 } 2005\end{array}$}} \\
\hline REMARK & & & & & & & \\
\hline НЕТАТМ & 1 & $\mathrm{H}$ & MOL & 0001 & 1.601 & -2.137 & $-1 \cdot 428$ \\
\hline HETATM & 2 & C & MOL & 0001 & -0.947 & -0.881 & -0.559 \\
\hline IETATM & 3 & C & MOL & 0001 & -2.890 & -0.159 & 0.766 \\
\hline ETATM & 4 & $\mathrm{~N}$ & MOL & 0001 & -1.164 & 1.465 & -0.013 \\
\hline HETATM & 5 & C & MOL & 0001 & -2.962 & -1.695 & 0.952 \\
\hline IETATM & 6 & C & MOL & 0001 & -0.577 & -1.453 & 0.806 \\
\hline HETATM & 7 & C & MOL & 0001 & -1.915 & 0.284 & -0.385 \\
\hline IETATM & 8 & $\mathrm{H}$ & MOL & 0001 & -2.495 & 0.273 & 1.672 \\
\hline HETATM & 9 & $\mathrm{H}$ & MOL & $0 \odot \odot 1$ & -0.360 & -0.685 & 1.520 \\
\hline HETATM & 10 & $\mathrm{H}$ & MOL & 0001 & -3.387 & -2.155 & 0.070 \\
\hline HETATM & 11 & 0 & MOL & 0001 & 0.282 & -3.488 & -0.115 \\
\hline HETATM & 12 & $\mathrm{H}$ & MOL & 0001 & -2.447 & 0.488 & -1.297 \\
\hline HETATM & 13 & $\mathrm{H}$ & MOL & $0 \odot \odot 1$ & 3. 681 & -1.789 & -1.6 \\
\hline HETATM & 14 & 0 & MOL & $0 \odot \odot 1$ & -4.256 & 0.268 & 0.682 \\
\hline HETATM & 15 & 0 & MOL & 0001 & -1.656 & -2.296 & 1.230 \\
\hline HETATM & 16 & $\mathrm{H}$ & MOL & 0001 & -3.598 & -1.902 & 1.795 \\
\hline HETATM & 17 & $\mathrm{H}$ & MOL & 0001 & -0.260 & 1.317 & ०. 398 \\
\hline HETATM & 18 & C & MOL & 0001 & -1.528 & 2.695 & -0.454 \\
\hline HETATM & 19 & 0 & MOL & 0001 & -2.533 & 2.903 & -1.134 \\
\hline HETATM & 20 & $\mathrm{~N}$ & MOL & 0001 & -0.676 & 3.689 & -0.085 \\
\hline HETATM & 21 & $\mathrm{H}$ & MOL & 0001 & 0.128 & 3.520 & 0.479 \\
\hline
\end{tabular}




\begin{tabular}{|c|c|c|c|c|c|c|c|c|}
\hline HETATM & 22 & $\mathrm{H}$ & MOL & 0001 & & -0.887 & 4.617 & -0.371 \\
\hline HETATM & 23 & $\mathrm{H}$ & MOL & 0001 & & -1.405 & -1.674 & -1.133 \\
\hline HETATM & 24 & C & MOL & $\odot \odot \odot 1$ & & 1.323 & -1.430 & $-\odot .666$ \\
\hline HETATM & 25 & $\mathrm{H}$ & MOL & 0001 & & -0.552 & -3.756 & 0.304 \\
\hline HETATM & 26 & C & MOL & 0001 & & 0.679 & -2.247 & 0.485 \\
\hline HETATM & 27 & $\mathrm{~N}$ & MOL & 0001 & & 2.521 & $-\odot .666$ & -0.319 \\
\hline HETATM & 28 & C & MOL & $\odot \odot \odot 1$ & & 4.702 & $\odot .842$ & 0.198 \\
\hline HETATM & 29 & C & MOL & $\odot \odot \odot 1$ & & 2.438 & 0.428 & $\odot .567$ \\
\hline HETATM & 30 & C & MOL & 0001 & & 3.700 & -0.960 & -0.935 \\
\hline HETATM & 31 & C & MOL & $\odot \odot \odot 1$ & & 4.818 & -0.261 & -0.711 \\
\hline HETATM & 32 & $\mathrm{~N}$ & MOL & 0001 & & 5.768 & 1.603 & 0.473 \\
\hline HETATM & 33 & 0 & MOL & $\odot \odot \odot 1$ & & 1.370 & 0.684 & 1.114 \\
\hline HETATM & 34 & $\mathrm{H}$ & MOL & 0001 & & 5.648 & 2.362 & 1.109 \\
\hline HETATM & 35 & $\mathrm{H}$ & MOL & $\odot \odot \odot 1$ & & 5.738 & -0.503 & -1.194 \\
\hline HETATM & 36 & $\mathrm{~N}$ & MOL & 0001 & & 3.572 & 1.139 & 0.775 \\
\hline HETATM & 37 & $\mathrm{H}$ & MOL & 0001 & & 6.663 & 1.441 & $\odot .069$ \\
\hline HETATM & 38 & H & MOL & $\odot \odot \odot 1$ & & 1.351 & -2.390 & 1.317 \\
\hline HETATM & 39 & 0 & MOL & $\odot \odot \odot 1$ & & $\odot .316$ & -0.535 & -1.165 \\
\hline HETATM & 40 & C & MOL & 0001 & & -4.827 & $\odot .684$ & -0.579 \\
\hline HETATM & 41 & $\mathrm{H}$ & MOL & $\odot \odot \odot 1$ & & -4.781 & -0.105 & -1.324 \\
\hline HETATM & 42 & $\mathrm{H}$ & MOL & 0001 & & -4.335 & 1.570 & -0.945 \\
\hline HETATM & 43 & $\mathrm{H}$ & MOL & 0001 & & -5.863 & 0.895 & -0.361 \\
\hline CONECT & 1 & 24 & & & & & & \\
\hline CONECT & 2 & 6 & 7 & 23 & 39 & & & \\
\hline CONECT & 3 & 8 & 14 & 5 & 7 & & & \\
\hline CONECT & 4 & 7 & 17 & 18 & & & & \\
\hline CONECT & 5 & 3 & 15 & 10 & 16 & & & \\
\hline CONECT & 6 & 2 & 15 & 26 & 9 & & & \\
\hline CONECT & 7 & 2 & 3 & 4 & 12 & & & \\
\hline CONECT & 8 & 3 & & & & & & \\
\hline CONECT & 9 & 6 & & & & & & \\
\hline CONECT & 10 & 5 & & & & & & \\
\hline CONECT & 11 & 26 & 25 & & & & & \\
\hline CONECT & 12 & 7 & & & & & & \\
\hline CONECT & 13 & 30 & & & & & & \\
\hline CONECT & 14 & 3 & 40 & & & & & \\
\hline CONECT & 15 & 5 & 6 & & & & & \\
\hline CONECT & 16 & 5 & & & & & & \\
\hline CONECT & 17 & 4 & & & & & & \\
\hline CONECT & 18 & 4 & 19 & 20 & & & & \\
\hline CONECT & 19 & 18 & & & & & & \\
\hline CONECT & 20 & 18 & 21 & 22 & & & & \\
\hline CONECT & 21 & 20 & & & & & & \\
\hline CONECT & 22 & 20 & & & & & & \\
\hline CONECT & 23 & 2 & & & & & & \\
\hline CONECT & 24 & 1 & 27 & 26 & 39 & & & \\
\hline CONECT & 25 & 11 & & & & & & \\
\hline CONECT & 26 & 6 & 11 & 24 & 38 & & & \\
\hline CONECT & 27 & 24 & 30 & 29 & & & & \\
\hline CONECT & 28 & 31 & 36 & 32 & & & & \\
\hline CONECT & 29 & 27 & 36 & 33 & & & & \\
\hline CONECT & 30 & 13 & 27 & 31 & & & & \\
\hline CONECT & 31 & 28 & 30 & 35 & & & & \\
\hline CONECT & 32 & 28 & 37 & 34 & & & & \\
\hline CONECT & 33 & 29 & & & & & & \\
\hline CONECT & 34 & 32 & & & & & & \\
\hline CONECT & 35 & 31 & & & & & & \\
\hline
\end{tabular}




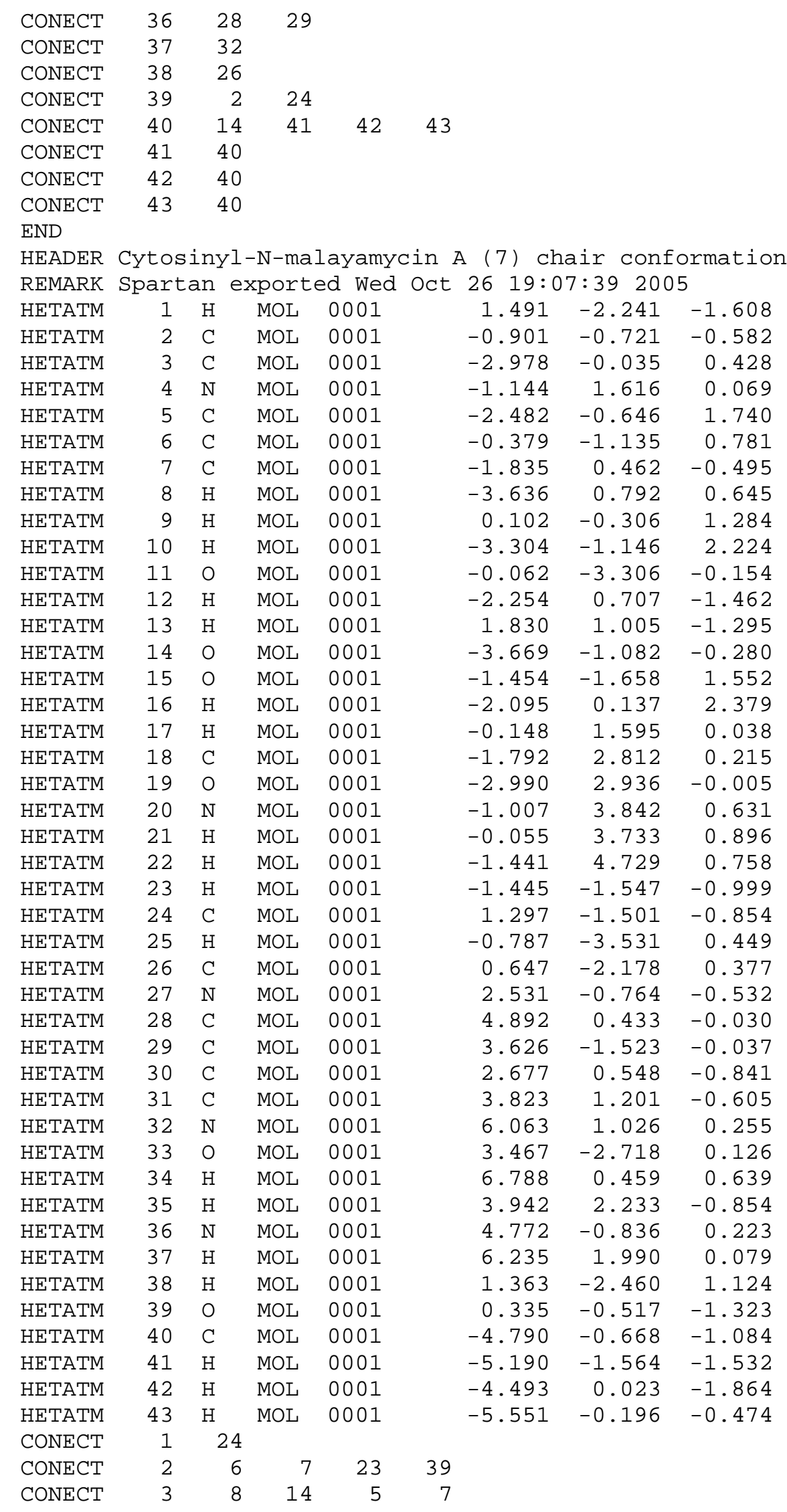




\begin{tabular}{|c|c|c|c|c|c|c|c|c|}
\hline CONECT & 4 & & 7 & 17 & 18 & & & \\
\hline CONECT & 5 & & 3 & 15 & 10 & 16 & & \\
\hline CONECT & 6 & & 2 & 15 & 26 & 9 & & \\
\hline CONECT & 7 & & 2 & 3 & 4 & 12 & & \\
\hline CONECT & 8 & & 3 & & & & & \\
\hline CONECT & 9 & & 6 & & & & & \\
\hline CONECT & 10 & & 5 & & & & & \\
\hline CONECT & 11 & 2 & & 25 & & & & \\
\hline CONECT & 12 & & 7 & & & & & \\
\hline CONECT & 13 & 3 & & & & & & \\
\hline CONECT & 14 & & 3 & 40 & & & & \\
\hline CONECT & 15 & & 5 & 6 & & & & \\
\hline CONECT & 16 & & 5 & & & & & \\
\hline CONECT & 17 & & 4 & & & & & \\
\hline CONECT & 18 & & 4 & 19 & 20 & & & \\
\hline CONECT & 19 & 18 & & & & & & \\
\hline CONECT & 20 & 18 & & 21 & 22 & & & \\
\hline CONECT & 21 & 2 & & & & & & \\
\hline CONECT & 22 & 2 & & & & & & \\
\hline CONECT & 23 & & 2 & & & & & \\
\hline CONECT & 24 & & 1 & 27 & 26 & 39 & & \\
\hline CONECT & 25 & 1 & 1 & & & & & \\
\hline CONECT & 26 & & 6 & 11 & 24 & 38 & & \\
\hline CONECT & 27 & 2 & & 30 & 29 & & & \\
\hline CONECT & 28 & 3 & & 36 & 32 & & & \\
\hline CONECT & 29 & 2 & & 36 & 33 & & & \\
\hline CONECT & 30 & $1:$ & & 27 & 31 & & & \\
\hline CONECT & 31 & $2 \varepsilon$ & & 30 & 35 & & & \\
\hline CONECT & 32 & 3 & 4 & 28 & 37 & & & \\
\hline CONECT & 33 & 2 & 9 & & & & & \\
\hline CONECT & 34 & 3 & 2 & & & & & \\
\hline CONECT & 35 & 3 & 1 & & & & & \\
\hline CONECT & 36 & 2 & 8 & 29 & & & & \\
\hline CONECT & 37 & 3 & 2 & & & & & \\
\hline CONECT & 38 & 2 & 6 & & & & & \\
\hline CONECT & 39 & & 2 & 24 & & & & \\
\hline CONECT & $4 \odot$ & 1 & 4 & 41 & 42 & 43 & & \\
\hline CONECT & 41 & 4 & 0 & & & & & \\
\hline CONECT & 42 & 4 & 0 & & & & & \\
\hline CONECT & 43 & 4 & $\odot$ & & & & & \\
\hline \multicolumn{9}{|l|}{ END } \\
\hline HEADER & \multicolumn{8}{|c|}{ Cytosinyl-N-malayamycin A (7) twistboat conformation } \\
\hline REMARK & \multicolumn{5}{|c|}{ Spartan exported Thu } & oct $2713: 2$ & $6: 34200$ & \\
\hline HETATM & 1 & $\mathrm{H}$ & MOL & & $\odot \odot 01$ & -1.667 & -1.899 & 2.049 \\
\hline HETATM & 2 & C & MOL & & 0001 & 0.875 & -0.825 & 0.705 \\
\hline HETATM & 3 & C & MOL & & $\odot \odot \odot 1$ & 2.567 & -0.210 & $-\odot .981$ \\
\hline HETATM & 4 & $\mathrm{~N}$ & MOL & & 0001 & 1.145 & 1.558 & 0.003 \\
\hline HETATM & 5 & C & MOL & & 0001 & 2.701 & -1.747 & -1.003 \\
\hline HETATM & 6 & C & MOL & & $\odot \odot \odot 1$ & $\odot .341$ & -1.595 & -0.493 \\
\hline HETATM & 7 & C & MOL & & 0001 & 1.817 & 0.303 & 0.284 \\
\hline HETATM & 8 & H & MOL & & 0001 & 1.974 & 0.063 & -1.843 \\
\hline HETATM & 9 & $\mathrm{H}$ & MOL & & $\odot \odot \odot 1$ & $-\odot . \odot 9 \odot$ & -0.930 & -1.231 \\
\hline HETATM & 10 & $\mathrm{H}$ & MOL & & 0001 & 3.248 & -2.106 & -0.142 \\
\hline HETATM & 11 & 0 & MOL & & $\odot \odot \odot 1$ & -0.104 & -3.396 & $1 . \odot \odot \odot$ \\
\hline HETATM & 12 & $\mathrm{H}$ & MOL & & 0001 & 2.504 & 0.517 & 1.083 \\
\hline HETATM & 13 & $\mathrm{H}$ & MOL & & 0001 & -1.567 & 1.157 & 0.760 \\
\hline HETATM & 14 & 0 & MOL & & 0001 & 3.841 & $\odot .387$ & -1.218 \\
\hline
\end{tabular}




\begin{tabular}{|c|c|c|c|c|c|c|c|c|}
\hline HETATM & 15 & 0 & MOL & $\odot \odot \odot 1$ & & 1.390 & -2.394 & -1.047 \\
\hline HETATM & 16 & $\mathrm{H}$ & MOL & $\odot \odot \odot 1$ & & 3.227 & -2.032 & -1.899 \\
\hline HETATM & 17 & $\mathrm{H}$ & MOL & 0001 & & 0.433 & 1.579 & -0.693 \\
\hline HETATM & 18 & C & MOL & $\odot \odot \odot 1$ & & 1.607 & 2.720 & 0.561 \\
\hline HETATM & 19 & 0 & MOL & 0001 & & 2.559 & 2.744 & 1.333 \\
\hline HETATM & 20 & $\mathrm{~N}$ & MOL & 0001 & & $\odot .932$ & 3.840 & $\odot .194$ \\
\hline HETATM & 21 & $\mathrm{H}$ & MOL & 0001 & & 0.159 & 3.839 & -0.431 \\
\hline HETATM & 22 & H & MOL & $\odot \odot \odot 1$ & & 1.242 & 4.708 & 0.571 \\
\hline HETATM & 23 & H & MOL & 0001 & & 1.417 & -1.532 & 1.312 \\
\hline HETATM & 24 & C & MOL & 0001 & & -1.360 & -1.404 & 1.147 \\
\hline HETATM & 25 & H & MOL & $\odot \odot \odot 1$ & & 0.541 & -3.880 & 0.463 \\
\hline HETATM & 26 & C & MOL & $\odot \odot \odot 1$ & & -0.749 & -2.423 & $\odot .166$ \\
\hline HETATM & 27 & $\mathrm{~N}$ & MOL & $\odot \odot \odot 1$ & & -2.491 & -0.662 & $\odot .562$ \\
\hline HETATM & 28 & C & MOL & 0001 & & -4.686 & 0.584 & -0.371 \\
\hline HETATM & 29 & C & MOL & $\odot \odot \odot 1$ & & -3.662 & -1.408 & 0.266 \\
\hline HETATM & 30 & C & MOL & $\odot \odot \odot 1$ & & -2.471 & 0.688 & $\odot .446$ \\
\hline HETATM & 31 & C & MOL & $\odot \odot \odot 1$ & & -3.530 & 1.363 & $-\odot .024$ \\
\hline HETATM & 32 & $\mathrm{~N}$ & MOL & $\odot \odot \odot 1$ & & -5.779 & 1.191 & -0.858 \\
\hline HETATM & 33 & 0 & MOL & $00 \odot 1$ & & -3.635 & -2.610 & 0.460 \\
\hline HETATM & 34 & H & MOL & $\odot \odot \odot 1$ & & -6.568 & 0.621 & -1.076 \\
\hline HETATM & 35 & $\mathrm{H}$ & MOL & 0001 & & -3.523 & 2.427 & -0.112 \\
\hline HETATM & 36 & $\mathrm{~N}$ & MOL & $\odot \odot \odot 1$ & & -4.719 & -0.708 & -0.226 \\
\hline HETATM & 37 & $\mathrm{H}$ & MOL & $\odot \odot \odot 1$ & & -5.835 & 2.176 & -0.987 \\
\hline HETATM & 38 & H & MOL & $\odot \odot \odot 1$ & & -1.481 & -2.861 & -0.485 \\
\hline HETATM & 39 & 0 & MOL & $\odot \odot \odot 1$ & & $-\odot .319$ & -0.433 & 1.414 \\
\hline HETATM & 40 & C & MOL & $\odot \odot \odot 1$ & & 4.745 & 0.604 & -0.108 \\
\hline HETATM & 41 & $\mathrm{H}$ & MOL & 0001 & & 4.877 & -0.290 & 0.492 \\
\hline HETATM & 42 & $\mathrm{H}$ & MOL & $\odot \odot \odot 1$ & & 4.400 & 1.416 & $\odot .513$ \\
\hline HETATM & 43 & $\mathrm{H}$ & MOL & $\odot \odot \odot 1$ & & 5.691 & $\odot .864$ & -0.557 \\
\hline CONECT & 1 & 24 & & & & & & \\
\hline CONECT & 2 & 6 & 7 & 23 & 39 & & & \\
\hline CONECT & 3 & 8 & 14 & 5 & 7 & & & \\
\hline CONECT & 4 & 7 & 17 & 18 & & & & \\
\hline CONECT & 5 & 3 & 15 & 10 & 16 & & & \\
\hline CONECT & 6 & 2 & 15 & 26 & 9 & & & \\
\hline CONECT & 7 & 2 & 3 & 4 & 12 & & & \\
\hline CONECT & 8 & 3 & & & & & & \\
\hline CONECT & 9 & 6 & & & & & & \\
\hline CONECT & 10 & 5 & & & & & & \\
\hline CONECT & 11 & 26 & 25 & & & & & \\
\hline CONECT & 12 & 7 & & & & & & \\
\hline CONECT & 13 & 30 & & & & & & \\
\hline CONECT & 14 & 3 & 40 & & & & & \\
\hline CONECT & 15 & 5 & 6 & & & & & \\
\hline CONECT & 16 & 5 & & & & & & \\
\hline CONECT & 17 & 4 & & & & & & \\
\hline CONECT & 18 & 4 & 19 & 20 & & & & \\
\hline CONECT & 19 & 18 & & & & & & \\
\hline CONECT & 20 & 18 & 21 & 22 & & & & \\
\hline CONECT & 21 & 20 & & & & & & \\
\hline CONECT & 22 & 20 & & & & & & \\
\hline CONECT & 23 & 2 & & & & & & \\
\hline CONECT & 24 & 1 & 27 & 26 & 39 & & & \\
\hline CONECT & 25 & 11 & & & & & & \\
\hline CONECT & 26 & 6 & 11 & 24 & 38 & & & \\
\hline CONECT & 27 & 24 & 30 & 29 & & & & \\
\hline CONECT & 28 & 31 & 36 & 32 & & & & \\
\hline
\end{tabular}




$\begin{array}{lrrrrr}\text { CONECT } & 29 & 27 & 36 & 33 & \\ \text { CONECT } & 30 & 13 & 27 & 31 & \\ \text { CONECT } & 31 & 28 & 30 & 35 & \\ \text { CONECT } & 32 & 28 & 37 & 34 & \\ \text { CONECT } & 33 & 29 & & & \\ \text { CONECT } & 34 & 32 & & & \\ \text { CONECT } & 35 & 31 & & & \\ \text { CONECT } & 36 & 28 & 29 & & \\ \text { CONECT } & 37 & 32 & & & \\ \text { CONECT } & 38 & 26 & & & \\ \text { CONECT } & 39 & 2 & 24 & & \\ \text { CONECT } & 40 & 14 & 41 & 42 & 43 \\ \text { CONECT } & 41 & 40 & & & \\ \text { CONECT } & 42 & 40 & & & \\ \text { CONECT } & 43 & 40 & & & \\ \text { END } & & & & & \end{array}$

HEADER epi-C6-methoxy-N-malayamycin (42) boat conformation REMARK Spartan exported Wed Oct 26 19:06:04 2005

\begin{tabular}{|c|c|c|c|c|c|c|c|}
\hline HETATM & 1 & $\mathrm{H}$ & MOL & 0001 & 1.926 & -2.300 & -1.136 \\
\hline HETATM & 2 & C & MOL & 0001 & -0.781 & -1.127 & -1.053 \\
\hline HETATM & 3 & C & MOL & 0001 & -3.007 & -0.373 & -0.274 \\
\hline HETATM & 4 & $\mathrm{~N}$ & MOL & 0001 & -1.210 & 1.219 & -0.980 \\
\hline HETATM & 5 & C & MOL & 0001 & -3.026 & -1.849 & 0.161 \\
\hline HETATM & 6 & C & MOL & 0001 & -0.682 & -1.395 & 0.445 \\
\hline HETATM & 7 & C & MOL & 0001 & -1.829 & -0.043 & -1.278 \\
\hline HETATM & 8 & 0 & MOL & 0001 & -2.832 & 0.401 & 0.920 \\
\hline HETATM & 9 & $\mathrm{H}$ & MOL & 0001 & -0.711 & -0.487 & 1.011 \\
\hline HETATM & 10 & $\mathrm{H}$ & MOL & 0001 & -3.225 & -2.488 & -0.690 \\
\hline HETATM & 11 & 0 & MOL & 0001 & 0.475 & -3.477 & 0.182 \\
\hline HETATM & 12 & $\mathrm{H}$ & MOL & 0001 & -2.218 & -0.062 & -2.286 \\
\hline HETATM & 13 & $\mathrm{H}$ & MOL & 0001 & 3.987 & -1.872 & -0.985 \\
\hline HETATM & 14 & $\mathrm{H}$ & MOL & 0001 & -3.944 & -0.127 & -0.753 \\
\hline HETATM & 15 & 0 & MOL & 0001 & -1.773 & -2.266 & 0.788 \\
\hline HETATM & 16 & $\mathrm{H}$ & MOL & 0001 & -3.800 & -1.992 & 0.898 \\
\hline HETATM & 17 & $\mathrm{H}$ & MOL & 0001 & -0.288 & 1.180 & -0.592 \\
\hline HETATM & 18 & C & MOL & 0001 & -1.950 & 2.344 & -0.879 \\
\hline HETATM & 19 & 0 & MOL & 0001 & -3.124 & 2.407 & -1.250 \\
\hline HETATM & 20 & $\mathrm{~N}$ & MOL & 0001 & -1.279 & 3.396 & $-\odot .342$ \\
\hline HETATM & 21 & $\mathrm{H}$ & MOL & 0001 & -0.376 & 3.284 & 0.067 \\
\hline HETATM & 22 & $\mathrm{H}$ & MOL & 0001 & -1.755 & 4.264 & -0.251 \\
\hline HETATM & 23 & $\mathrm{H}$ & MOL & 0001 & -1.061 & -2.047 & -1.545 \\
\hline HETATM & 24 & C & MOL & 0001 & 1.484 & -1.478 & -0.601 \\
\hline HETATM & 25 & $\mathrm{H}$ & MOL & 0001 & -0.423 & -3.728 & 0.455 \\
\hline HETATM & 26 & C & MOL & 0001 & 0.658 & -2.103 & 0.556 \\
\hline HETATM & 27 & $\mathrm{~N}$ & MOL & 0001 & 2.571 & -0.582 & -0.206 \\
\hline HETATM & 28 & C & MOL & 0001 & 4.578 & 1.116 & 0.417 \\
\hline HETATM & 29 & C & MOL & 0001 & 2.291 & 0.661 & $0.40 \odot$ \\
\hline HETATM & 30 & C & MOL & 0001 & 3.855 & -0.926 & -0.503 \\
\hline HETATM & 31 & C & MOL & 0001 & 4.893 & -0.136 & -0.211 \\
\hline HETATM & 32 & $\mathrm{~N}$ & MOL & 0001 & 5.556 & 1.971 & 0.740 \\
\hline HETATM & 33 & 0 & MOL & 0001 & 1.131 & 0.963 & $\odot .650$ \\
\hline HETATM & 34 & $\mathrm{H}$ & MOL & 0001 & 5.297 & 2.833 & 1.170 \\
\hline HETATM & 35 & $\mathrm{H}$ & MOL & 0001 & 5.896 & -0.416 & -0.443 \\
\hline HETATM & 36 & $\mathrm{~N}$ & MOL & 0001 & 3.351 & 1.458 & 0.684 \\
\hline HETATM & 37 & $\mathrm{H}$ & MOL & 0001 & 6.517 & 1.781 & 0.563 \\
\hline
\end{tabular}




\begin{tabular}{|c|c|c|c|c|c|c|c|c|}
\hline HETATM & 38 & $\mathrm{H}$ & MOL & 0001 & & 1.146 & -2.003 & 1.512 \\
\hline HETATM & 39 & 0 & MOL & 0001 & & 0.563 & -0.775 & -1.451 \\
\hline HETATM & 40 & C & MOL & $0 \odot \odot 1$ & & -4.013 & 1.065 & 1.420 \\
\hline HETATM & 41 & $\mathrm{H}$ & MOL & $\odot \odot \odot 1$ & & -4.785 & $\odot .351$ & 1.690 \\
\hline HETATM & 42 & $\mathrm{H}$ & MOL & $\odot \odot \odot 1$ & & -4.381 & 1.755 & 0.677 \\
\hline HETATM & 43 & $\mathrm{H}$ & MOL & $\odot \odot \odot 1$ & & -3.704 & 1.601 & 2.304 \\
\hline CONECT & 1 & 24 & & & & & & \\
\hline CONECT & 2 & 6 & 7 & 23 & 39 & & & \\
\hline CONECT & 3 & 8 & 14 & 5 & 7 & & & \\
\hline CONECT & 4 & 7 & 17 & 18 & & & & \\
\hline CONECT & 5 & 3 & 15 & 10 & 16 & & & \\
\hline CONECT & 6 & 2 & 15 & 26 & 9 & & & \\
\hline CONECT & 7 & 2 & 3 & 4 & 12 & & & \\
\hline CONECT & 8 & 3 & 40 & & & & & \\
\hline CONECT & 9 & 6 & & & & & & \\
\hline CONECT & 10 & 5 & & & & & & \\
\hline CONECT & 11 & 26 & 25 & & & & & \\
\hline CONECT & 12 & 7 & & & & & & \\
\hline CONECT & 13 & 30 & & & & & & \\
\hline CONECT & 14 & 3 & & & & & & \\
\hline CONECT & 15 & 5 & 6 & & & & & \\
\hline CONECT & 16 & 5 & & & & & & \\
\hline CONECT & 17 & 4 & & & & & & \\
\hline CONECT & 18 & 4 & 19 & 20 & & & & \\
\hline CONECT & 19 & 18 & & & & & & \\
\hline CONECT & 20 & 18 & 21 & 22 & & & & \\
\hline CONECT & 21 & 20 & & & & & & \\
\hline CONECT & 22 & 20 & & & & & & \\
\hline CONECT & 23 & 2 & & & & & & \\
\hline CONECT & 24 & 1 & 27 & 26 & 39 & & & \\
\hline CONECT & 25 & 11 & & & & & & \\
\hline CONECT & 26 & 6 & 11 & 24 & 38 & & & \\
\hline CONECT & 27 & 24 & 30 & 29 & & & & \\
\hline CONECT & 28 & 31 & 36 & 32 & & & & \\
\hline CONECT & 29 & 27 & 36 & 33 & & & & \\
\hline CONECT & 30 & 13 & 27 & 31 & & & & \\
\hline CONECT & 31 & 28 & 30 & 35 & & & & \\
\hline CONECT & 32 & 28 & 37 & 34 & & & & \\
\hline CONECT & 33 & 29 & & & & & & \\
\hline CONECT & 34 & 32 & & & & & & \\
\hline CONECT & 35 & 31 & & & & & & \\
\hline CONECT & 36 & 28 & 29 & & & & & \\
\hline CONECT & 37 & 32 & & & & & & \\
\hline CONECT & 38 & 26 & & & & & & \\
\hline CONECT & 39 & 2 & 24 & & & & & \\
\hline CONECT & 40 & 8 & 41 & 42 & 43 & & & \\
\hline CONECT & 41 & $4 \odot$ & & & & & & \\
\hline CONECT & 42 & $4 \odot$ & & & & & & \\
\hline CONECT & 43 & $4 \odot$ & & & & & & \\
\hline \multicolumn{9}{|l|}{ END } \\
\hline \multirow{2}{*}{\multicolumn{9}{|c|}{$\begin{array}{l}\text { HEADER epi-C6-methoxy-N-malayamycin (42) chair conformation } \\
\text { REMARK spartan exported wed Oct } 2619: 05: 462005\end{array}$}} \\
\hline & & & & & & & & \\
\hline HETATM & 1 & $\mathrm{H}$ & MOL & $\odot \odot \odot 1$ & & -1.692 & -2.214 & 1.796 \\
\hline HETATM & 2 & C & MOL & $\odot \odot \odot 1$ & & 0.878 & $-\odot .925$ & 0.778 \\
\hline HETATM & 3 & C & MOL & $\odot \odot \odot 1$ & & 3.065 & $-\odot .5 \odot 9$ & -0.133 \\
\hline HETATM & 4 & $\mathrm{~N}$ & MOL & $\odot \odot \odot 1$ & & 1.490 & 1.298 & $-\odot .247$ \\
\hline HETATM & 5 & $\mathrm{C}$ & MOL & $\odot \odot \odot 1$ & & 2.608 & -1.261 & -1.404 \\
\hline
\end{tabular}




\begin{tabular}{|c|c|c|c|c|c|c|c|c|}
\hline HETATM & 6 & C & MOL & 0001 & & $\odot .401$ & -1.451 & -0.560 \\
\hline HETATM & 7 & C & MOL & $\odot \odot 01$ & & 1.893 & $\odot .192$ & 0.611 \\
\hline HETATM & 8 & 0 & MOL & $\odot \odot \odot 1$ & & 3.999 & $\odot .448$ & -0.652 \\
\hline HETATM & 9 & $\mathrm{H}$ & MOL & 0001 & & 0.035 & -0.647 & -1.181 \\
\hline HETATM & 10 & $\mathrm{H}$ & MOL & 0001 & & 3.421 & -1.866 & -1.770 \\
\hline HETATM & 11 & 0 & MOL & 0001 & & -0.118 & -3.500 & 0.543 \\
\hline HETATM & 12 & $\mathrm{H}$ & MOL & $\odot \odot \odot 1$ & & 2.205 & $\odot .560$ & 1.581 \\
\hline HETATM & 13 & $\mathrm{H}$ & MOL & $\odot \odot \odot 1$ & & -1.597 & 1.059 & 1.105 \\
\hline HETATM & 14 & $\mathrm{H}$ & MOL & 0001 & & 3.551 & -1.195 & 0.550 \\
\hline HETATM & 15 & 0 & MOL & $\odot \odot \odot 1$ & & 1.492 & -2.147 & -1.174 \\
\hline HETATM & 16 & $\mathrm{H}$ & MOL & 0001 & & 2.352 & -0.527 & -2.155 \\
\hline HETATM & 17 & $\mathrm{H}$ & MOL & 0001 & & 2.148 & 1.492 & -0.975 \\
\hline HETATM & 18 & C & MOL & $0 \odot 01$ & & 0.696 & 2.318 & 0.197 \\
\hline HETATM & 19 & 0 & MOL & $\odot \odot \odot 1$ & & 0.140 & 2.313 & 1.289 \\
\hline HETATM & 20 & $\mathrm{~N}$ & MOL & 0001 & & 0.574 & 3.350 & -0.681 \\
\hline HETATM & 21 & $\mathrm{H}$ & MOL & 0001 & & $\odot .937$ & 3.324 & -1.607 \\
\hline HETATM & 22 & $\mathrm{H}$ & MOL & 0001 & & 0.011 & 4.125 & -0.409 \\
\hline HETATM & 23 & H & MOL & $\odot \odot \odot 1$ & & 1.374 & -1.746 & 1.274 \\
\hline HETATM & 24 & C & MOL & 0001 & & -1.370 & -1.553 & 1.014 \\
\hline HETATM & 25 & $\mathrm{H}$ & MOL & $\odot \odot \odot 1$ & & 0.554 & -3.881 & -0.041 \\
\hline HETATM & 26 & C & MOL & 0001 & & -0.719 & -2.375 & -0.120 \\
\hline HETATM & 27 & $\mathrm{~N}$ & MOL & 0001 & & -2.492 & -0.723 & 0.554 \\
\hline HETATM & 28 & C & MOL & 0001 & & -4.690 & 0.647 & -0.160 \\
\hline HETATM & 29 & C & MOL & $\odot \odot \odot 1$ & & -3.641 & -1.413 & 0.095 \\
\hline HETATM & 30 & C & MOL & $0 \odot \odot 1$ & & -2.486 & 0.628 & 0.700 \\
\hline HETATM & 31 & C & MOL & 0001 & & -3.556 & 1.359 & 0.347 \\
\hline HETATM & 32 & $\mathrm{~N}$ & MOL & $0 \odot \odot 1$ & & -5.791 & 1.319 & -0.539 \\
\hline HETATM & 33 & 0 & MOL & 0001 & & -3.594 & -2.631 & 0.051 \\
\hline HETATM & 34 & H & MOL & $\odot \odot \odot 1$ & & -6.566 & 0.788 & $-\odot .872$ \\
\hline HETATM & 35 & $\mathrm{H}$ & MOL & $\odot \odot \odot 1$ & & -3.561 & 2.420 & 0.459 \\
\hline HETATM & 36 & $\mathrm{~N}$ & MOL & $\odot \odot \odot 1$ & & -4.706 & $-\odot .651$ & -0.270 \\
\hline HETATM & 37 & $\mathrm{H}$ & MOL & 0001 & & -5.863 & 2.308 & -0.470 \\
\hline HETATM & 38 & $\mathrm{H}$ & MOL & $\odot \odot \odot 1$ & & -1.424 & -2.671 & $-\odot .873$ \\
\hline HETATM & 39 & 0 & MOL & 0001 & & -0.344 & -0.647 & 1.490 \\
\hline HETATM & 40 & C & MOL & $\odot \odot \odot 1$ & & 4.917 & 1.031 & $\odot .297$ \\
\hline HETATM & 41 & $\mathrm{H}$ & MOL & $\odot \odot \odot 1$ & & 5.485 & $\odot .261$ & $\odot .805$ \\
\hline HETATM & 42 & $\mathrm{H}$ & MOL & $\odot \odot \odot 1$ & & 4.397 & 1.635 & 1.030 \\
\hline HETATM & 43 & $\mathrm{H}$ & MOL & 0001 & & 5.588 & 1.655 & -0.273 \\
\hline CONECT & 1 & 24 & & & & & & \\
\hline CONECT & 2 & 6 & 7 & 23 & 39 & & & \\
\hline CONECT & 3 & 8 & 14 & 5 & 7 & & & \\
\hline CONECT & 4 & 7 & 17 & 18 & & & & \\
\hline CONECT & 5 & 3 & 15 & 10 & 16 & & & \\
\hline CONECT & 6 & 2 & 15 & 26 & 9 & & & \\
\hline CONECT & 7 & 2 & 3 & 4 & 12 & & & \\
\hline CONECT & 8 & 3 & 40 & & & & & \\
\hline CONECT & 9 & 6 & & & & & & \\
\hline CONECT & 10 & 5 & & & & & & \\
\hline CONECT & 11 & 26 & 25 & & & & & \\
\hline CONECT & 12 & 7 & & & & & & \\
\hline CONECT & 13 & 30 & & & & & & \\
\hline CONECT & 14 & 3 & & & & & & \\
\hline CONECT & 15 & 5 & 6 & & & & & \\
\hline CONECT & 16 & 5 & & & & & & \\
\hline CONECT & 17 & 4 & & & & & & \\
\hline CONECT & 18 & 4 & 19 & 20 & & & & \\
\hline CONECT & 19 & 18 & & & & & & \\
\hline
\end{tabular}




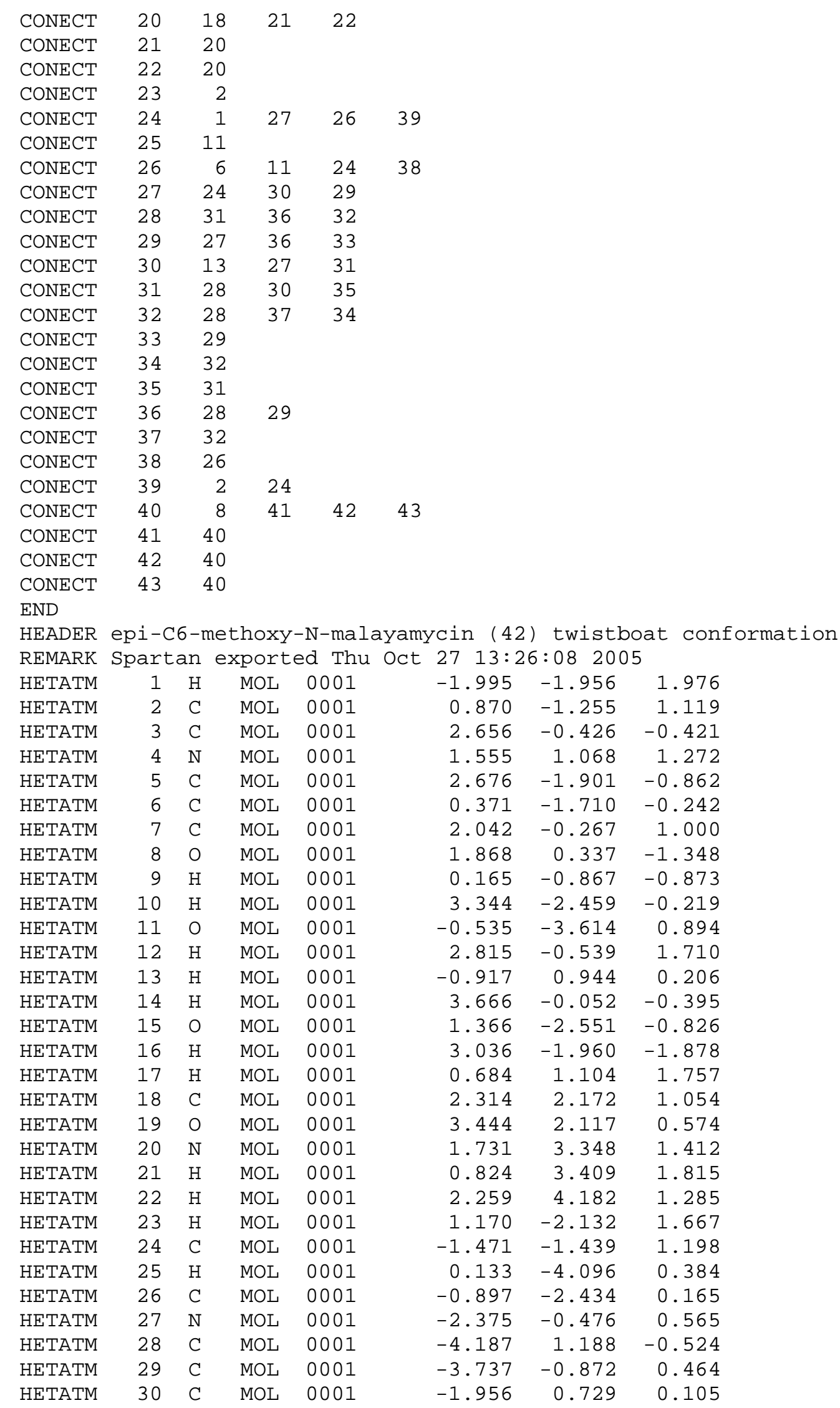




\begin{tabular}{|c|c|c|c|c|c|c|c|c|}
\hline HETATM & 31 & C & MOL & $0 \odot 01$ & & -2.811 & 1.596 & -0.455 \\
\hline HETATM & 32 & $\mathrm{~N}$ & MOL & 0001 & & -5.101 & 2.017 & -1.056 \\
\hline HETATM & 33 & 0 & MOL & 0001 & & -4.037 & -1.981 & 0.862 \\
\hline HETATM & 34 & $\mathrm{H}$ & MOL & 0001 & & -6.047 & 1.703 & -1.090 \\
\hline HETATM & 35 & $\mathrm{H}$ & MOL & $\odot \odot \odot 1$ & & -2.485 & 2.541 & $-\odot .828$ \\
\hline HETATM & 36 & $\mathrm{~N}$ & MOL & $\odot \odot \odot 1$ & & -4.592 & $\odot .033$ & $-\odot .087$ \\
\hline HETATM & 37 & $\mathrm{H}$ & MOL & 0001 & & -4.867 & 2.914 & -1.416 \\
\hline HETATM & 38 & $\mathrm{H}$ & MOL & 0001 & & -1.601 & -2.638 & -0.624 \\
\hline HETATM & 39 & 0 & MOL & 0001 & & -0.330 & $-\odot .707$ & 1.723 \\
\hline HETATM & 40 & C & MOL & 0001 & & 2.614 & 1.090 & -2.331 \\
\hline HETATM & 41 & $\mathrm{H}$ & MOL & 0001 & & 3.175 & 0.435 & -2.988 \\
\hline HETATM & 42 & $\mathrm{H}$ & MOL & 0001 & & 3.281 & 1.778 & -1.833 \\
\hline HETATM & 43 & $\mathrm{H}$ & MOL & 0001 & & 1.884 & 1.631 & -2.914 \\
\hline CONECT & 1 & 24 & & & & & & \\
\hline CONECT & 2 & 6 & 7 & 23 & 39 & & & \\
\hline CONECT & 3 & 8 & 14 & 5 & 7 & & & \\
\hline CONECT & 4 & 7 & 17 & 18 & & & & \\
\hline CONECT & 5 & 3 & 15 & 10 & 16 & & & \\
\hline CONECT & 6 & 2 & 15 & 26 & 9 & & & \\
\hline CONECT & 7 & 2 & 3 & 4 & 12 & & & \\
\hline CONECT & 8 & 3 & 40 & & & & & \\
\hline CONECT & 9 & 6 & & & & & & \\
\hline CONECT & 10 & 5 & & & & & & \\
\hline CONECT & 11 & 26 & 25 & & & & & \\
\hline CONECT & 12 & 7 & & & & & & \\
\hline CONECT & 13 & 30 & & & & & & \\
\hline CONECT & 14 & 3 & & & & & & \\
\hline CONECT & 15 & 5 & 6 & & & & & \\
\hline CONECT & 16 & 5 & & & & & & \\
\hline CONECT & 17 & 4 & & & & & & \\
\hline CONECT & 18 & 4 & 19 & 20 & & & & \\
\hline CONECT & 19 & 18 & & & & & & \\
\hline CONECT & 20 & 18 & 21 & 22 & & & & \\
\hline CONECT & 21 & 20 & & & & & & \\
\hline CONECT & 22 & 20 & & & & & & \\
\hline CONECT & 23 & 2 & & & & & & \\
\hline CONECT & 24 & 1 & 27 & 26 & 39 & & & \\
\hline CONECT & 25 & 11 & & & & & & \\
\hline CONECT & 26 & 6 & 11 & 24 & 38 & & & \\
\hline CONECT & 27 & 24 & 30 & 29 & & & & \\
\hline CONECT & 28 & 31 & 36 & 32 & & & & \\
\hline CONECT & 29 & 27 & 36 & 33 & & & & \\
\hline CONECT & 30 & 13 & 27 & 31 & & & & \\
\hline CONECT & 31 & 28 & 30 & 35 & & & & \\
\hline CONECT & 32 & 28 & 37 & 34 & & & & \\
\hline CONECT & 33 & 29 & & & & & & \\
\hline CONECT & 34 & 32 & & & & & & \\
\hline CONECT & 35 & 31 & & & & & & \\
\hline CONECT & 36 & 28 & 29 & & & & & \\
\hline CONECT & 37 & 32 & & & & & & \\
\hline CONECT & 38 & 26 & & & & & & \\
\hline CONECT & 39 & 2 & 24 & & & & & \\
\hline CONECT & 40 & 8 & 41 & 42 & 43 & & & \\
\hline CONECT & 41 & 40 & & & & & & \\
\hline CONECT & 42 & 40 & & & & & & \\
\hline CONECT & 43 & 40 & & & & & & \\
\hline END & & & & & & & & \\
\hline
\end{tabular}




\begin{tabular}{|c|c|c|c|c|c|c|c|c|}
\hline REMARK & Sp & $\operatorname{an} e$ & xport & ed Wed & $0 c t$ & $2615:$ & $: 3320$ & \\
\hline HETATM & 1 & $\mathrm{H}$ & MOL & $\odot \odot \odot 1$ & & 1.180 & -2.240 & -1.286 \\
\hline HETATM & 2 & C & MOL & $\odot \odot \odot 1$ & & -1.367 & -0.817 & -0.827 \\
\hline HETATM & 3 & C & MOL & $\odot \odot \odot 1$ & & -3.400 & $\odot . \odot 9 \odot$ & 0.234 \\
\hline HETATM & 4 & $\mathrm{~N}$ & MOL & $\odot \odot \odot 1$ & & -1.565 & 1.556 & -0.522 \\
\hline HETATM & 5 & C & MOL & $\odot \odot \odot 1$ & & -3.558 & -1.389 & 0.590 \\
\hline HETATM & 6 & C & MOL & $0 \odot 01$ & & -1.155 & -1.226 & 0.627 \\
\hline HETATM & 7 & C & MOL & $0 \odot 01$ & & -2.311 & 0.375 & -0.861 \\
\hline HETATM & 8 & $\mathrm{~F}$ & MOL & $\odot \odot \odot 1$ & & -2.998 & 0.722 & 1.429 \\
\hline HETATM & 9 & $\mathrm{H}$ & MOL & 0001 & & -1.021 & -0.378 & 1.264 \\
\hline HETATM & 10 & $\mathrm{H}$ & MOL & $\odot \odot \odot 1$ & & -3.951 & -1.930 & -0.260 \\
\hline HETATM & 11 & 0 & MOL & $\odot \odot \odot 1$ & & $-\odot .272$ & -3.380 & 0.064 \\
\hline HETATM & 12 & $\mathrm{H}$ & MOL & $\odot \odot \odot 1$ & & -2.800 & 0.482 & -1.818 \\
\hline HETATM & 13 & $\mathrm{H}$ & MOL & $\odot \odot \odot 1$ & & 3.286 & -1.999 & -1.329 \\
\hline HETATM & 14 & $\mathrm{H}$ & MOL & 0001 & & -4.340 & 0.520 & -0.064 \\
\hline HETATM & 15 & 0 & MOL & $\odot \odot \odot 1$ & & -2.301 & -2.003 & 1.014 \\
\hline HETATM & 16 & $\mathrm{H}$ & MOL & $\odot \odot \odot 1$ & & -4.240 & -1.479 & 1.419 \\
\hline HETATM & 17 & $\mathrm{H}$ & MOL & $\odot \odot \odot 1$ & & $-\odot .626$ & 1.427 & -0.194 \\
\hline HETATM & 18 & C & MOL & 0001 & & -2.221 & 2.735 & -0.386 \\
\hline HETATM & 19 & 0 & MOL & 0001 & & -3.402 & 2.859 & -0.700 \\
\hline HETATM & 20 & $\mathrm{~N}$ & MOL & 0001 & & -1.462 & 3.745 & 0.110 \\
\hline HETATM & 21 & $\mathrm{H}$ & MOL & 0001 & & $-\odot .538$ & 3.593 & $\odot .450$ \\
\hline HETATM & 22 & $\mathrm{H}$ & MOL & 0001 & & -1.892 & 4.632 & 0.245 \\
\hline HETATM & 23 & $\mathrm{H}$ & MOL & $0 \odot \odot 1$ & & -1.793 & -1.661 & -1.351 \\
\hline HETATM & 24 & C & MOL & $\odot \odot \odot 1$ & & 0.878 & -1.432 & $-\odot .643$ \\
\hline HETATM & 25 & $\mathrm{H}$ & MOL & $\odot \odot \odot 1$ & & -1.151 & -3.577 & 0.422 \\
\hline HETATM & 26 & C & MOL & 0001 & & 0.100 & -2.076 & $\odot .537$ \\
\hline HETATM & 27 & $\mathrm{~N}$ & MOL & $0 \odot \odot 1$ & & 2.079 & $-\odot .681$ & -0.289 \\
\hline HETATM & 28 & C & MOL & $\odot \odot \odot 1$ & & 4.283 & 0.780 & 0.262 \\
\hline HETATM & 29 & C & MOL & 0001 & & 1.975 & 0.515 & 0.453 \\
\hline HETATM & 30 & C & MOL & 0001 & & 3.290 & -1.098 & -0.753 \\
\hline HETATM & 31 & C & MOL & $\odot \odot \odot 1$ & & 4.419 & -0.426 & -0.505 \\
\hline HETATM & 32 & $\mathrm{~N}$ & MOL & 0001 & & 5.361 & 1.517 & 0.551 \\
\hline HETATM & 33 & 0 & MOL & $0 \odot \odot 1$ & & 0.876 & 0.880 & $\odot .852$ \\
\hline HETATM & 34 & $\mathrm{H}$ & MOL & $\odot \odot \odot 1$ & & 5.227 & 2.351 & 1.083 \\
\hline HETATM & 35 & $\mathrm{H}$ & MOL & 0001 & & 5.364 & -0.762 & -0.868 \\
\hline HETATM & 36 & $\mathrm{~N}$ & MOL & $\odot \odot \odot 1$ & & 3.124 & 1.193 & 0.690 \\
\hline HETATM & 37 & $\mathrm{H}$ & MOL & $\odot \odot \odot 1$ & & 6.278 & 1.269 & $\odot .256$ \\
\hline HETATM & 38 & $\mathrm{H}$ & MOL & 0001 & & 0.684 & -2.121 & 1.442 \\
\hline HETATM & 39 & 0 & MOL & 0001 & & -0.041 & $-\odot .569$ & -1.337 \\
\hline CONECT & 1 & 24 & & & & & & \\
\hline CONECT & 2 & 6 & 7 & 23 & 39 & & & \\
\hline CONECT & 3 & 8 & 14 & 5 & 7 & & & \\
\hline CONECT & 4 & 7 & 17 & 18 & & & & \\
\hline CONECT & 5 & 3 & 15 & 10 & 16 & & & \\
\hline CONECT & 6 & 2 & 15 & 26 & 9 & & & \\
\hline CONECT & 7 & 2 & 3 & 4 & 12 & & & \\
\hline CONECT & 8 & 3 & 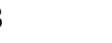 & & & & & \\
\hline CONECT & 9 & 6 & ; & & & & & \\
\hline CONECT & 10 & 5 & ; & & & & & \\
\hline CONECT & 11 & 26 & 25 & & & & & \\
\hline CONECT & 12 & 7 & 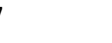 & & & & & \\
\hline CONECT & 13 & 30 & & & & & & \\
\hline CONECT & 14 & 3 & 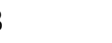 & & & & & \\
\hline CONECT & 15 & 5 & 6 & & & & & \\
\hline CONECT & 16 & 5 & & & & & & \\
\hline
\end{tabular}




\begin{tabular}{|c|c|c|c|c|c|c|c|}
\hline CONECT & 17 & & 4 & & & & \\
\hline CONECT & 18 & & 19 & 20 & & & \\
\hline CONECT & 19 & $1 \varepsilon$ & 8 & & & & \\
\hline CONECT & 20 & $1 \varepsilon$ & 21 & 22 & & & \\
\hline CONECT & 21 & $2 C$ & 20 & & & & \\
\hline CONECT & 22 & $2 C$ & $\odot$ & & & & \\
\hline CONECT & 23 & & 2 & & & & \\
\hline CONECT & 24 & & 27 & 26 & 39 & & \\
\hline CONECT & 25 & 11 & 1 & & & & \\
\hline CONECT & 26 & & 6 & 24 & 38 & & \\
\hline CONECT & 27 & 24 & 30 & 29 & & & \\
\hline CONECT & 28 & 31 & 36 & 32 & & & \\
\hline CONECT & 29 & 27 & 36 & 33 & & & \\
\hline CONECT & 30 & 13 & 27 & 31 & & & \\
\hline CONECT & 31 & $2 \varepsilon$ & 36 & 35 & & & \\
\hline CONECT & 32 & $2 \varepsilon$ & 37 & 34 & & & \\
\hline CONECT & 33 & 2 & 9 & & & & \\
\hline CONECT & 34 & 3 & 32 & & & & \\
\hline CONECT & 35 & 3 & 31 & & & & \\
\hline CONECT & 36 & $2 \varepsilon$ & 8 & & & & \\
\hline CONECT & 37 & 3 & 32 & & & & \\
\hline CONECT & 38 & 2 & 6 & & & & \\
\hline CONECT & 39 & & 24 & & & & \\
\hline END & & & & & & & \\
\hline HEADER & $\mathrm{C} 6-\mathrm{fl}$ & uor & $\mathrm{O}-\mathrm{N}-\mathrm{ma}$ & alayamy & $\operatorname{cin}(43)$ cha & ir confo & rmation \\
\hline REMARK & Spart & an & export & ted Wed & Oct $26 \quad 19: \odot$ & $6: 52 \quad 20 \odot$ & \\
\hline HETATM & 1 & $\mathrm{H}$ & MOL & $0 \odot \odot 1$ & 0.913 & -2.278 & -1.759 \\
\hline HETATM & 2 & C & MOL & $\odot \odot \odot 1$ & -1.470 & -0.611 & -0.855 \\
\hline HETATM & 3 & C & MOL & $\odot \odot \odot 1$ & -3.595 & 0.108 & $-\odot .042$ \\
\hline HETATM & 4 & $\mathrm{~N}$ & MOL & 0001 & -1.810 & 1.700 & 0.109 \\
\hline HETATM & 5 & C & MOL & $\odot \odot \odot 1$ & -3.301 & -0.644 & 1.270 \\
\hline HETATM & 6 & C & MOL & $\odot \odot \odot 1$ & -1.120 & -1.169 & 0.509 \\
\hline HETATM & 7 & C & MOL & $\odot \odot \odot 1$ & -2.329 & $\odot .639$ & -0.747 \\
\hline HETATM & 8 & $\mathrm{~F}$ & MOL & 0001 & -4.390 & 1.211 & 0.347 \\
\hline HETATM & 9 & $\mathrm{H}$ & MOL & $\odot \odot \odot 1$ & $-\odot .662$ & -0.414 & 1.131 \\
\hline HETATM & 10 & $\mathrm{H}$ & MOL & $0 \odot \odot 1$ & -4.202 & -1.113 & 1.632 \\
\hline HETATM & 11 & 0 & MOL & $\odot \odot \odot 1$ & $-\odot .87 \odot$ & -3.293 & -0.547 \\
\hline HETATM & 12 & $\mathrm{H}$ & MOL & 0001 & -2.571 & 1.025 & -1.728 \\
\hline HETATM & 13 & $\mathrm{H}$ & MOL & $\odot \odot \odot 1$ & 1.285 & $\odot .987$ & -1.122 \\
\hline HETATM & 14 & $\mathrm{H}$ & MOL & $\odot \odot \odot 1$ & -4.164 & -0.503 & -0.722 \\
\hline HETATM & 15 & 0 & MOL & $\odot \odot \odot 1$ & -2.322 & -1.686 & 1.098 \\
\hline HETATM & 16 & $\mathrm{H}$ & MOL & $\odot \odot \odot 1$ & -2.961 & 0.074 & 2.003 \\
\hline HETATM & 17 & $\mathrm{H}$ & MOL & $\odot \odot \odot 1$ & -2.479 & 2.047 & 0.764 \\
\hline HETATM & 18 & C & MOL & $\odot \odot \odot 1$ & -0.856 & 2.582 & -0.335 \\
\hline HETATM & 19 & 0 & MOL & $\odot \odot \odot 1$ & -0.255 & 2.443 & -1.391 \\
\hline HETATM & 20 & $\mathrm{~N}$ & MOL & $\odot \odot \odot 1$ & -0.636 & 3.628 & 0.505 \\
\hline HETATM & 21 & $\mathrm{H}$ & MOL & $\odot \odot \odot 1$ & -1.055 & 3.705 & 1.404 \\
\hline HETATM & 22 & $\mathrm{H}$ & MOL & 0001 & 0.034 & 4.310 & 0.229 \\
\hline HETATM & 23 & $\mathrm{H}$ & MOL & $\odot \odot \odot 1$ & -2.059 & -1.367 & -1.354 \\
\hline HETATM & 24 & C & MOL & $\odot \odot \odot 1$ & 0.668 & -1.561 & -0.998 \\
\hline HETATM & 25 & $\mathrm{H}$ & MOL & $\odot \odot \odot 1$ & -1.583 & -3.598 & 0.032 \\
\hline HETATM & 26 & C & MOL & $\odot \odot \odot 1$ & -0.134 & -2.257 & 0.124 \\
\hline HETATM & 27 & $\mathrm{~N}$ & MOL & 0001 & 1.883 & $-\odot .897$ & -0.511 \\
\hline HETATM & 28 & C & MOL & $\odot \odot \odot 1$ & 4.239 & 0.142 & 0.257 \\
\hline HETATM & 29 & C & MOL & 0001 & 2.900 & -1.742 & 0.001 \\
\hline HETATM & 30 & C & MOL & $0 \odot \odot 1$ & 2.086 & 0.436 & -0.679 \\
\hline HETATM & 31 & C & MOL & $\odot \odot \odot 1$ & 3.242 & 1.005 & -0.301 \\
\hline
\end{tabular}




\begin{tabular}{|c|c|c|c|c|c|c|c|c|}
\hline HETATM & 32 & $\mathrm{~N}$ & MOL & 0001 & & 5.416 & 0.649 & 0.662 \\
\hline HETATM & 33 & 0 & MOL & $\odot \odot \odot 1$ & & 2.667 & -2.938 & $\odot .065$ \\
\hline HETATM & 34 & $\mathrm{H}$ & MOL & $\odot \odot \odot 1$ & & 5.640 & 1.614 & 0.578 \\
\hline HETATM & 35 & $\mathrm{H}$ & MOL & $\odot \odot \odot 1$ & & 3.411 & 2.051 & -0.431 \\
\hline HETATM & 36 & $\mathrm{~N}$ & MOL & $\odot \odot \odot 1$ & & 4.055 & -1.141 & $\odot .39 \odot$ \\
\hline HETATM & 37 & $\mathrm{H}$ & MOL & $\odot \odot \odot 1$ & & 6.092 & $\odot .015$ & 1.030 \\
\hline HETATM & 38 & $\mathrm{H}$ & MOL & $\odot \odot \odot 1$ & & $\odot .493$ & -2.637 & $\odot .905$ \\
\hline HETATM & 39 & 0 & MOL & $\odot \odot \odot 1$ & & -0.198 & -0.523 & -1.523 \\
\hline CONECT & 1 & 24 & & & & & & \\
\hline CONECT & 2 & 6 & 7 & 23 & 39 & & & \\
\hline CONECT & 3 & 8 & 14 & 5 & 7 & & & \\
\hline CONECT & 4 & 7 & 17 & 18 & & & & \\
\hline CONECT & 5 & 3 & 15 & 10 & 16 & & & \\
\hline CONECT & 6 & 2 & 15 & 26 & 9 & & & \\
\hline CONECT & 7 & 2 & 3 & 4 & 12 & & & \\
\hline CONECT & 8 & 3 & & & & & & \\
\hline CONECT & 9 & 6 & & & & & & \\
\hline CONECT & 10 & 5 & & & & & & \\
\hline CONECT & 11 & 26 & 25 & & & & & \\
\hline CONECT & 12 & 7 & & & & & & \\
\hline CONECT & 13 & 30 & & & & & & \\
\hline CONECT & 14 & 3 & & & & & & \\
\hline CONECT & 15 & 5 & 6 & & & & & \\
\hline CONECT & 16 & 5 & & & & & & \\
\hline CONECT & 17 & 4 & & & & & & \\
\hline CONECT & 18 & 4 & 19 & 20 & & & & \\
\hline CONECT & 19 & 18 & & & & & & \\
\hline CONECT & 20 & 18 & 21 & 22 & & & & \\
\hline CONECT & 21 & 20 & & & & & & \\
\hline CONECT & 22 & 20 & & & & & & \\
\hline CONECT & 23 & 2 & & & & & & \\
\hline CONECT & 24 & 1 & 27 & 26 & 39 & & & \\
\hline CONECT & 25 & 11 & & & & & & \\
\hline CONECT & 26 & 6 & 11 & 24 & 38 & & & \\
\hline CONECT & 27 & 24 & 30 & 29 & & & & \\
\hline CONECT & 28 & 31 & 36 & 32 & & & & \\
\hline CONECT & 29 & 27 & 36 & 33 & & & & \\
\hline CONECT & 30 & 13 & 27 & 31 & & & & \\
\hline CONECT & 31 & 28 & 30 & 35 & & & & \\
\hline CONECT & 32 & 34 & 28 & 37 & & & & \\
\hline CONECT & 33 & 29 & & & & & & \\
\hline CONECT & 34 & 32 & & & & & & \\
\hline CONECT & 35 & 31 & & & & & & \\
\hline CONECT & 36 & 28 & 29 & & & & & \\
\hline CONECT & 37 & 32 & & & & & & \\
\hline CONECT & 38 & 26 & & & & & & \\
\hline CONECT & 39 & 2 & 24 & & & & & \\
\hline \multicolumn{9}{|l|}{ END } \\
\hline \multicolumn{9}{|c|}{ HEADER C6-fluoro-N-malayamycin (43) } \\
\hline REMARK & Spart & an e> & xporte & ed Thu & Oct & $27 \quad 13: 4$ & $9: 36 \quad 2005$ & \\
\hline HETATM & 1 & $\mathrm{H}$ & MOL & 0001 & & -1.350 & -1.652 & 2.154 \\
\hline HETATM & 2 & C & MOL & $\odot \odot \odot 1$ & & 1.309 & -0.947 & 0.807 \\
\hline HETATM & 3 & C & MOL & $\odot \odot \odot 1$ & & 2.802 & -0.246 & -1.063 \\
\hline HETATM & 4 & $\mathrm{~N}$ & MOL & 0001 & & 1.889 & 1.401 & 0.558 \\
\hline HETATM & 5 & C & MOL & $\odot \odot \odot 1$ & & 2.841 & -1.743 & -1.373 \\
\hline HETATM & 6 & C & MOL & 0001 & & 0.654 & -1.595 & -0.402 \\
\hline HETATM & 7 & C & MOL & $\odot \odot \odot 1$ & & 2.398 & 0.056 & $\odot .398$ \\
\hline
\end{tabular}




\begin{tabular}{|c|c|c|c|c|c|c|c|c|}
\hline HETATM & 8 & $\mathrm{~F}$ & MOL & $\odot \odot \odot 1$ & & 1.846 & 0.344 & -1.922 \\
\hline HETATM & 9 & $\mathrm{H}$ & MOL & $\odot \odot \odot 1$ & & $\odot .297$ & -0.849 & -1.087 \\
\hline HETATM & 10 & $\mathrm{H}$ & MOL & $\odot \odot \odot 1$ & & 3.657 & -2.198 & -0.830 \\
\hline HETATM & 11 & 0 & MOL & $\odot \odot \odot 1$ & & 0.042 & -3.366 & 1.089 \\
\hline HETATM & 12 & H & MOL & 0001 & & 3.283 & -0.094 & 1.004 \\
\hline HETATM & 13 & H & MOL & 0001 & & $-\odot .756$ & 1.099 & -0.007 \\
\hline HETATM & 14 & $\mathrm{H}$ & MOL & $\odot \odot \odot 1$ & & 3.750 & $\odot .213$ & -1.274 \\
\hline HETATM & 15 & 0 & MOL & $\odot \odot \odot 1$ & & 1.611 & -2.451 & -1.023 \\
\hline HETATM & 16 & H & MOL & $\odot \odot \odot 1$ & & 3.001 & -1.872 & -2.432 \\
\hline HETATM & 17 & $\mathrm{H}$ & MOL & $\odot \odot \odot 1$ & & 1.089 & 1.482 & 1.149 \\
\hline HETATM & 18 & C & MOL & $\odot \odot \odot 1$ & & 2.675 & 2.471 & 0.236 \\
\hline HETATM & 19 & 0 & MOL & $\odot \odot \odot 1$ & & 3.787 & 2.328 & -0.256 \\
\hline HETATM & 20 & $\mathrm{~N}$ & MOL & $\odot \odot \odot 1$ & & 2.134 & 3.685 & 0.510 \\
\hline HETATM & 21 & $\mathrm{H}$ & MOL & $\odot \odot \odot 1$ & & 1.216 & 3.805 & $\odot .875$ \\
\hline HETATM & 22 & H & MOL & $\odot \odot \odot 1$ & & 2.674 & 4.492 & $\odot .291$ \\
\hline HETATM & 23 & H & MOL & $\odot \odot \odot 1$ & & 1.727 & -1.734 & 1.410 \\
\hline HETATM & 24 & C & MOL & $\odot \odot \odot 1$ & & -0.981 & -1.219 & 1.247 \\
\hline HETATM & 25 & H & MOL & $\odot \odot \odot 1$ & & 0.625 & -3.916 & 0.546 \\
\hline HETATM & 26 & C & MOL & $\odot \odot \odot 1$ & & -0.495 & -2.317 & $\odot .275$ \\
\hline HETATM & 27 & $\mathrm{~N}$ & MOL & $\odot \odot \odot 1$ & & -2.027 & -0.395 & $\odot .637$ \\
\hline HETATM & 28 & C & MOL & $\odot \odot \odot 1$ & & -4.094 & 0.996 & -0.379 \\
\hline HETATM & 29 & C & MOL & 0001 & & -3.349 & -0.913 & 0.725 \\
\hline HETATM & 30 & C & MOL & 0001 & & -1.775 & 0.790 & 0.028 \\
\hline HETATM & 31 & C & MOL & $\odot \odot \odot 1$ & & -2.762 & 1.522 & -0.503 \\
\hline HETATM & 32 & $\mathrm{~N}$ & MOL & $\odot \odot \odot 1$ & & -5.132 & 1.689 & -0.871 \\
\hline HETATM & 33 & 0 & MOL & $\odot \odot \odot 1$ & & -3.494 & -2.002 & 1.247 \\
\hline HETATM & 34 & H & MOL & $\odot \odot \odot 1$ & & -6.043 & 1.295 & -0.770 \\
\hline HETATM & 35 & H & MOL & $\odot \odot \odot 1$ & & -2.569 & 2.450 & -0.993 \\
\hline HETATM & 36 & $\mathrm{~N}$ & MOL & 0001 & & -4.339 & -0.142 & 0.201 \\
\hline HETATM & 37 & $\mathrm{H}$ & MOL & $\odot \odot \odot 1$ & & -5.024 & 2.563 & -1.333 \\
\hline HETATM & 38 & H & MOL & $\odot \odot \odot 1$ & & -1.287 & -2.658 & -0.369 \\
\hline HETATM & 39 & 0 & MOL & 0001 & & 0.173 & -0.368 & 1.498 \\
\hline CONECT & 1 & 24 & & & & & & \\
\hline CONECT & 2 & 6 & 7 & 23 & 39 & & & \\
\hline CONECT & 3 & 8 & 14 & 5 & 7 & & & \\
\hline CONECT & 4 & 7 & 17 & 18 & & & & \\
\hline CONECT & 5 & 3 & 15 & 10 & 16 & & & \\
\hline CONECT & 6 & 2 & 15 & 26 & 9 & & & \\
\hline CONECT & 7 & 2 & 3 & 4 & 12 & & & \\
\hline CONECT & 8 & 3 & & & & & & \\
\hline CONECT & 9 & 6 & & & & & & \\
\hline CONECT & 10 & 5 & & & & & & \\
\hline CONECT & 11 & 26 & 25 & & & & & \\
\hline CONECT & 12 & 7 & & & & & & \\
\hline CONECT & 13 & 30 & & & & & & \\
\hline CONECT & 14 & 3 & & & & & & \\
\hline CONECT & 15 & 5 & 6 & & & & & \\
\hline CONECT & 16 & 5 & & & & & & \\
\hline CONECT & 17 & 4 & & & & & & \\
\hline CONECT & 18 & 4 & 19 & 20 & & & & \\
\hline CONECT & 19 & 18 & & & & & & \\
\hline CONECT & 20 & 18 & 21 & 22 & & & & \\
\hline CONECT & 21 & 20 & & & & & & \\
\hline CONECT & 22 & 20 & & & & & & \\
\hline CONECT & 23 & 2 & & & & & & \\
\hline CONECT & 24 & 1 & 27 & 26 & 39 & & & \\
\hline CONECT & 25 & 11 & & & & & & \\
\hline
\end{tabular}




$\begin{array}{lrrrrr}\text { CONECT } & 26 & 6 & 11 & 24 & 38 \\ \text { CONECT } & 27 & 24 & 30 & 29 & \\ \text { CONECT } & 28 & 31 & 36 & 32 & \\ \text { CONECT } & 29 & 27 & 36 & 33 & \\ \text { CONECT } & 30 & 13 & 27 & 31 & \\ \text { CONECT } & 31 & 28 & 30 & 35 & \\ \text { CONECT } & 32 & 28 & 37 & 34 & \\ \text { CONECT } & 33 & 29 & & & \\ \text { CONECT } & 34 & 32 & & \\ \text { CONECT } & 35 & 31 & & \\ \text { CONECT } & 36 & 28 & 29 & \\ \text { CONECT } & 37 & 32 & & \\ \text { CONECT } & 38 & 26 & & \\ \text { CONECT } & 39 & 2 & 24 & \\ \text { END } & & & & & \end{array}$

